Prepared in cooperation with the Upper Elkhorn Natural Resources District and the Lower Elkhorn Natural Resources District

\title{
Trends in Streamflow Characteristics of Selected Sites in the Elkhorn River, Salt Creek, and Lower Platte River Basins, Eastern Nebraska, 1928-2004, and Evaluation of Streamflows in Relation to Instream-Flow Criteria, 1953-2004
}

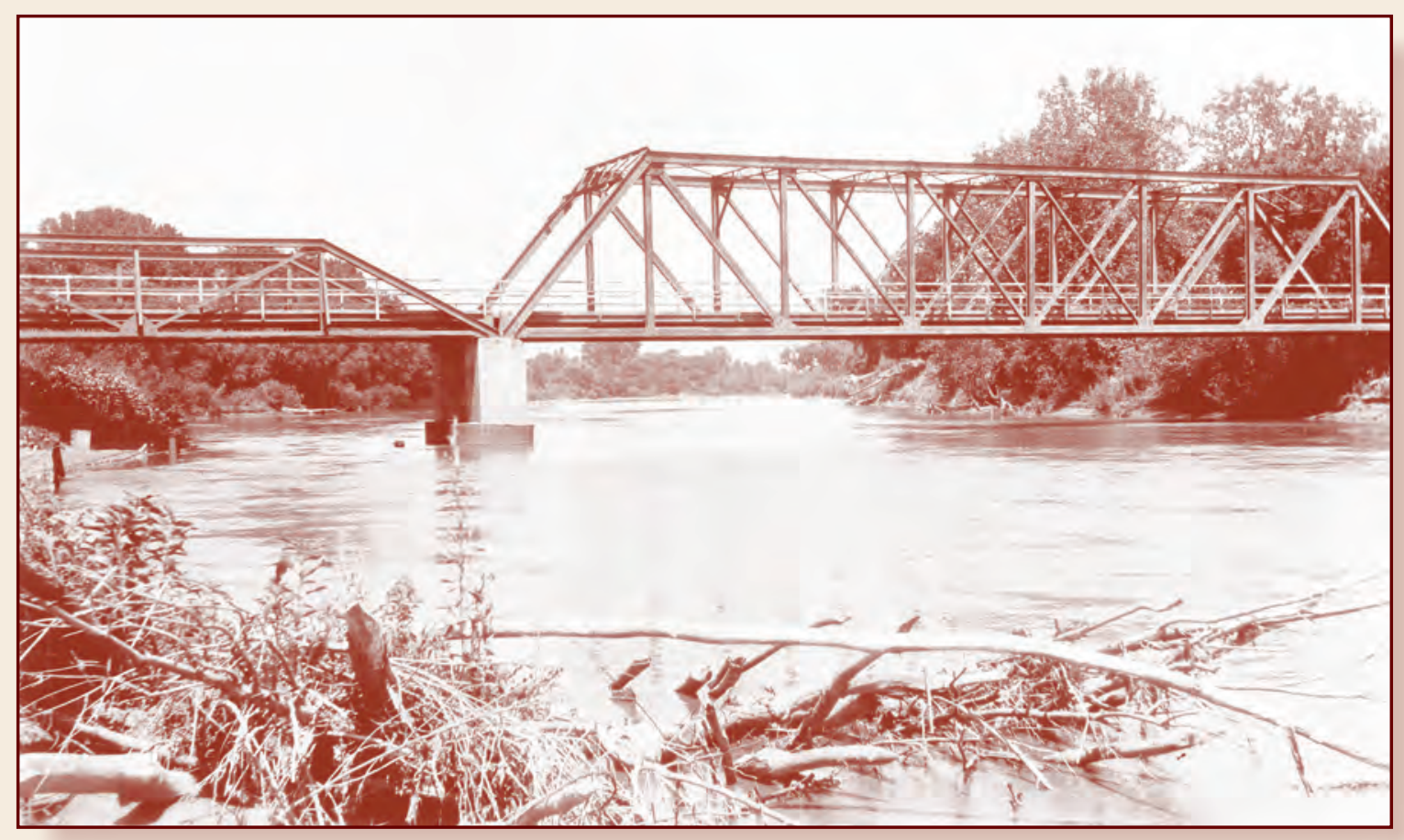

Scientific Investigations Report 2009-5011 
Cover. Bridge over the Elkhorn River at Waterloo, Nebraska (photograph taken by the U.S. Geological Survey, 1928). 


\section{Trends in Streamflow Characteristics of Selected Sites in the Elkhorn River, Salt Creek, and Lower Platte River Basins, Eastern Nebraska, 1928-2004, and Evaluation of Streamflows in Relation to Instream-Flow Criteria, 1953-2004}

By Benjamin J. Dietsch, Julie A. Godberson, and Gregory V. Steele

Prepared in cooperation with the Upper Elkhorn Natural Resources District and the Lower Elkhorn Natural Resources District

Scientific Investigations Report 2009-5011 


\title{
U.S. Department of the Interior \\ KEN SALAZAR, Secretary
}

\author{
U.S. Geological Survey \\ Suzette M. Kimball, Acting Director
}

U.S. Geological Survey, Reston, Virginia: 2009

For more information on the USGS - the Federal source for science about the Earth, its natural and living resources, natural hazards, and the environment, visit http://www.usgs.gov or call 1-888-ASK-USGS

For an overview of USGS information products, including maps, imagery, and publications, visit http://www.usgs.gov/pubprod

To order this and other USGS information products, visit http://store.usgs.gov

Any use of trade, product, or firm names is for descriptive purposes only and does not imply endorsement by the U.S. Government.

Although this report is in the public domain, permission must be secured from the individual copyright owners to reproduce any copyrighted materials contained within this report.

Suggested citation:

Dietsch, B.J, Godberson, J.A., and Steele, G.V., 2009, Trends in streamflow characteristics of selected sites in the Elkhorn River, Salt Creek, and Lower Platte River basins, eastern Nebraska, 1928-2004, and evaluation of streamflows in relation to instream-flow criteria, 1953-2004: U.S. Geological Survey Scientific Investigations Report 2009-5011, 93 p. with appendixes. 


\section{Contents}

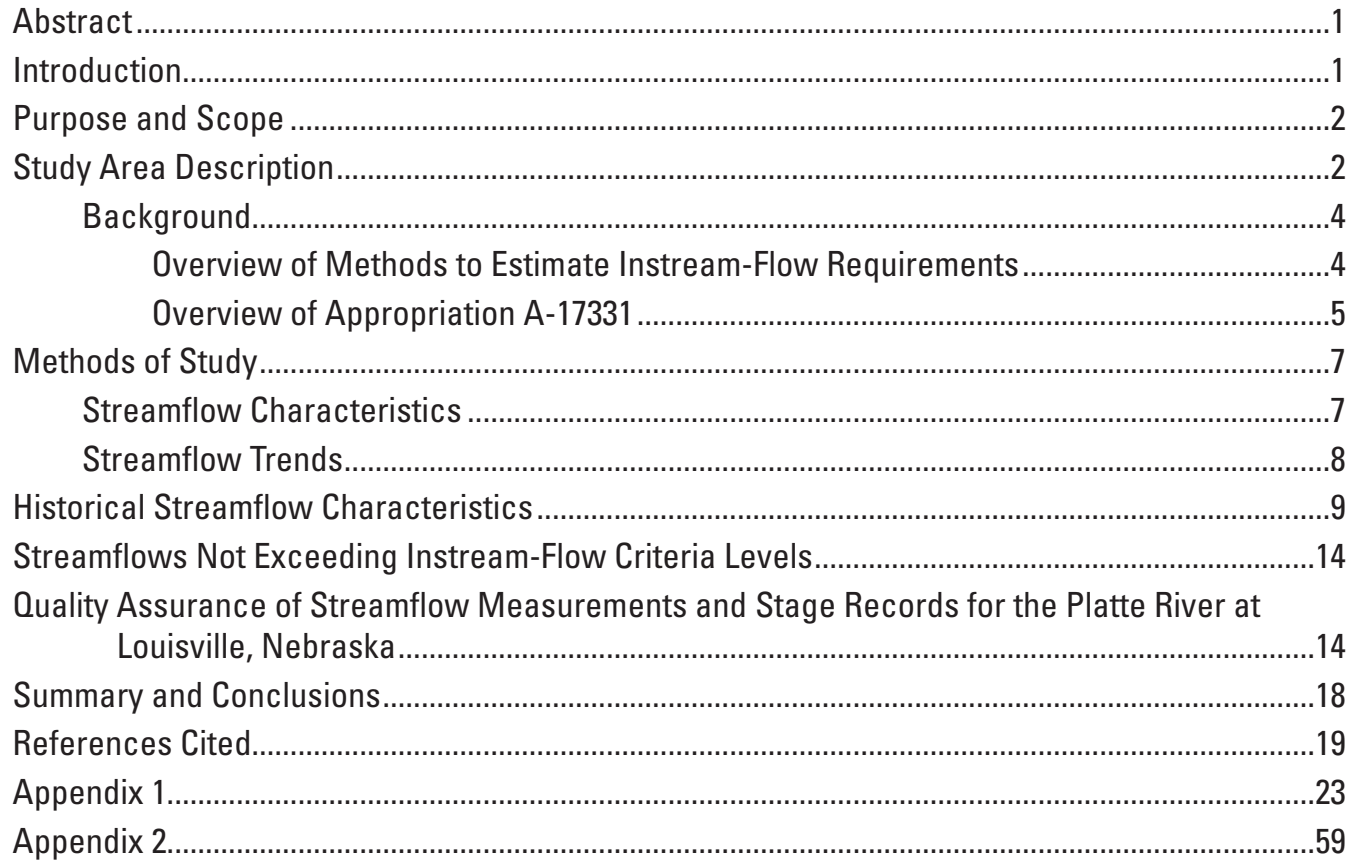

\section{Figures}

1. Map showing study area and streamflow-gaging stations in the Elkhorn River, Salt Creek, and Platte River basins, Nebraska

2-3. Graphs showing:

2. Non-exceedance duration hydrograph for Platte River at Louisville, Nebraska,

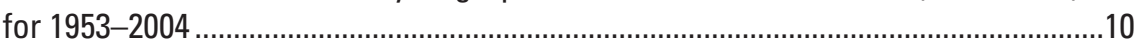

3. Annual mean streamflow and 7-day low flow of Platte River at Louisville, Nebraska, for 1954-2004.

\section{Tables}

1. Tennant standard-setting method for estimating instream-flow requirements ................5

2. Summary of Nebraska Game and Parks Commission applications for instream-flow appropriations, Platte River, Nebraska

3. Platte River instream flows appropriated by the Nebraska Department of Natural Resources to the Nebraska Game and Parks Commission on June 26, 1998...................7

4. Periods of operation of U.S. Geological Survey and Nebraska Department of Natural Resources streamflow-gaging stations in the Elkhorn River, Salt Creek, and Platte River basins, Nebraska.

5. Flow duration table for Platte River at Louisville, Nebraska, for water years 1953 through 2004 
6. Summary statistics for annual mean streamflow expressed as a percentage of the annual mean streamflow at the U.S. Geological Survey streamflow-gaging station on Platte River at Louisville, Nebraska (station 06805500), for water years 1954 through 2004

7. Median slope of significant low-flow trends at selected sites in the Elkhorn River, Salt Creek, and Platte River basins, Nebraska.....

8. Summary by water year of intervals in which daily mean streamflow at the U.S. Geological Survey streamflow-gaging station on the Platte River at Louisville, Nebraska (station 06805500), did not satisfy instream-flow criteria levels, 1953-2004.

9. Comparison by period of intervals in which daily mean streamflow at the U.S. Geological Survey streamflow-gaging station on the Platte River at Louisville, Nebraska (station 06805500), did not exceed instream-flow criteria levels, 1953-2004

10. Summary of discharge measurements from water years 1985 through 2004 for U.S. Geological Survey streamflow-gaging station on the Platte River at Louisville, Nebraska (station 06805500)

\section{Conversion Factors, Abbreviations, and Datums}

\begin{tabular}{|c|c|c|}
\hline Multiply & By & To obtain \\
\hline \multicolumn{3}{|c|}{ Length } \\
\hline inch (in.) & 2.54 & centimeter $(\mathrm{cm})$ \\
\hline foot (ft) & 0.3048 & meter $(\mathrm{m})$ \\
\hline mile (mi) & 1.609 & kilometer $(\mathrm{km})$ \\
\hline \multicolumn{3}{|c|}{ Area } \\
\hline acre & 4,047 & square meter $\left(\mathrm{m}^{2}\right)$ \\
\hline acre & 0.4047 & hectare (ha) \\
\hline square foot $\left(\mathrm{ft}^{2}\right)$ & 0.09290 & square meter $\left(\mathrm{m}^{2}\right)$ \\
\hline square inch $\left(\mathrm{in}^{2}\right)$ & 6.452 & square centimeter $\left(\mathrm{cm}^{2}\right)$ \\
\hline square mile $\left(\mathrm{mi}^{2}\right)$ & 2.590 & square kilometer $\left(\mathrm{km}^{2}\right)$ \\
\hline \multicolumn{3}{|c|}{ Volume } \\
\hline gallon (gal) & 3.785 & liter $(\mathrm{L})$ \\
\hline acre-foot (acre-ft) & 1,233 & cubic meter $\left(\mathrm{m}^{3}\right)$ \\
\hline \multicolumn{3}{|c|}{ Flow rate } \\
\hline acre-foot per day (acre-ft/d) & 0.01427 & cubic meter per second $\left(\mathrm{m}^{3} / \mathrm{s}\right)$ \\
\hline acre-foot per year (acre-ft/yr) & 1,233 & cubic meter per year $\left(\mathrm{m}^{3} / \mathrm{yr}\right)$ \\
\hline cubic foot per second $\left(\mathrm{ft}^{3} / \mathrm{s}\right)$ & 0.02832 & cubic meter per second $\left(\mathrm{m}^{3} / \mathrm{s}\right)$ \\
\hline foot per second (ft/s) & 0.3048 & meter per second $(\mathrm{m} / \mathrm{s})$ \\
\hline
\end{tabular}

Horizontal coordinate information is referenced to the North American Datum of 1983 (NAD 83). 


\title{
Trends in Streamflow Characteristics of Selected Sites in the Elkhorn River, Salt Creek, and Lower Platte River Basins, Eastern Nebraska, 1928-2004, and Evaluation of Streamflows in Relation to Instream-Flow Criteria, 1953-2004
}

\author{
By Benjamin J. Dietsch, Julie A. Godberson, and Gregory V. Steele
}

\section{Abstract}

The Nebraska Department of Natural Resources approved instream-flow appropriations on the Platte River to maintain fish communities, whooping crane roost habitat, and wet meadows used by several wild bird species. In the lower Platte River region, the Nebraska Game and Parks Commission owns an appropriation filed to maintain streamflow for fish communities between the Platte River confluence with the Elkhorn River and the mouth of the Platte River. Because Elkhorn River flow is an integral part of the flow in the reach addressed by this appropriation, the Upper Elkhorn and Lower Elkhorn Natural Resources Districts are involved in overall management of anthropogenic effects on the availability of surface water for instream requirements.

The Physical Habitat Simulation System (PHABSIM) and other estimation methodologies were used previously to determine instream requirements for Platte River biota, which led to the filing of five water appropriations applications with the Nebraska Department of Natural Resources in 1993 by the Nebraska Game and Parks Commission. One of these requested instream-flow appropriations of 3,700 cubic feet per second was for the reach from the Elkhorn River to the mouth of the Platte River. Four appropriations were granted with modifications in 1998, by the Nebraska Department of Natural Resources.

Daily streamflow data for the periods of record were summarized for 17 streamflow-gaging stations in Nebraska to evaluate streamflow characteristics, including low-flow intervals for consecutive durations of $1,3,7,14,30,60$, and 183 days. Temporal trends in selected streamflow statistics were not adjusted for variability in precipitation. Results indicated significant positive temporal trends in annual flow for the period of record at eight streamflow-gaging stations Platte River near Duncan (06774000), Platte River at North Bend (06796000), Elkhorn River at Neligh (06798500), Logan
Creek near Uehling (06799500), Maple Creek near Nickerson (06800000), Elkhorn River at Waterloo (06800500), Salt Creek at Greenwood (06803555), and Platte River at Louisville (06805500). In general, sites in the Elkhorn River Basin upstream from Norfolk showed fewer significant trends than did sites downstream from Norfolk and sites in the Platte River and Salt Creek basins, where trends in low flows also were positive.

Historical Platte River streamflow records for the streamflow-gaging station at Louisville, Nebraska, were used to determine the number of days per water year (Sept. 30 to Oct. 1) when flows failed to satisfy the minimum criteria of the instream-flow appropriation prior to its filing in 1993. Before 1993, the median number of days the criteria were not satisfied was about 120 days per water year. During 1993 through 2004, daily mean flows at Louisville, Nebraska, have failed to satisfy the criteria for 638 days total (median value equals 21.5 days per year). Most of these low-flow intervals occurred in summer through early fall. For water years 1953 through 2004, of the discrete intervals when flow was less that the criteria levels, 61 percent were 3 days or greater in duration, and 38 percent were 7 days or greater in duration. The median duration of intervals of flow less than the criteria levels was 4 consecutive days during 1953 through 2004.

\section{Introduction}

Instream-flow protection is a complex legislative and technical issue that is implemented in various ways throughout the United States. The Nebraska Department of Natural Resources (NDNR) approved appropriations on the Platte River to provide instream-flow protection primarily to maintain fish communities, whooping crane roost habitat, and wet meadows used by several migratory bird species. Instreamflow Appropriation A-17331, filed and owned by the Nebraska 
Game and Parks Commission (NGPC), was established to maintain streamflow for fish communities in the Platte River between its confluence with the Elkhorn River and the mouth of the Platte. The Elkhorn River flow is an integral part of the streamflow addressed in this appropriation. As such, the Upper Elkhorn Natural Resources District (UENRD) and the Lower Elkhorn Natural Resources District (LENRD) are collecting data and developing water-management strategies to assist with water-resources planning. Of particular interest are the effect of anthropogenic stresses on the availability of surface-water resources, and the interaction of ground water and surface water in the Elkhorn River basin. Specifically, the UENRD and the LENRD are concerned with the effects of precipitation, surface-water withdrawals, ground-water withdrawals, streambed-elevation changes, and tile drains on the availability of surface water and the long-term effects of these processes (R. Wozniak, Lower Elkhorn Natural Resources District, oral commun., 2005).

In 2005 the U.S. Geological Survey (USGS), the UENRD, and the LENRD began a cooperative study to investigate instream flow as it pertains to Appropriation A-17331. This study was undertaken to (1) review and assess the methods used to determine the flows granted by NDNR in Appropriation A-17331, (2) to provide information on temporal trends in streamflow at 17 streamflow-gaging stations located on the lower Platte River or within the basins of the Elkhorn River and Salt Creek, (3) to assess the sufficiency of historical streamflow data to meet the requirements of A-17331, and (4) to evaluate the adequacy of the streamflow records for the streamflow-gaging station at Louisville, Nebraska.

\section{Purpose and Scope}

The primary purpose of this report is to present the trends in streamflow characteristics from 1928 to 2004 at selected sites in the Elkhorn River, Salt Creek, and lower Platte River basins. Also, this report describes streamflows in relation to instream-flow criteria for 1953 to 2004. Findings for seven study objectives are presented: (1) review the technical literature to determine how Appropriation A-17331 was applied to maintain fish communities, and to identify the methods used to establish Appropriation A-17331 and similar flow appropriations for the maintenance of flow for fish and wildlife; (2) review the input conditions and applicability of the Physical Habitat Simulation System (PHABSIM) model used in establishment of Appropriation A-17331; (3) analyze longterm records of streamflow at streamflow-gaging stations to detect annual and seasonal variations, including those during the months of June, July, and August, and to determine longterm streamflow trends; (4) compute 1- and 7-day low flows for each year to determine long-term surface-water trends; (5) summarize historical occurrences of daily streamflow values in the Elkhorn River basin and the Platte River at Louisville, Nebraska, that would be considered insufficient, as defined by Appropriation A-17331, to maintain the fish community between the Platte River confluences with the Elkhorn River and the Missouri River; (6) determine the number of days that flows in the Elkhorn River basin and at the Platte River at Louisville streamflow-gaging station would be considered insufficient, as defined by Appropriation A-17331, since November 1993 (the beginning date of the appropriation) and identify the dates when junior water rights would have been suspended as a result of water shortages for Appropriation A-17331; and (7) provide quality-assurance data on the accuracy of the streamflow measurements at the Platte River at Louisville streamflow-gaging station and its effect on the adequacy of the Louisville streamflow-gaging station for management of the instream-flow appropriation.

The scope of the study includes the available streamflow data at 17 streamflow-gaging stations in the Elkhorn River, Platte River, and Salt Creek basins.

\section{Study Area Description}

Confluences with two principal tributaries of the Platte River - the Elkhorn River and Salt Creek - are located within the reach defined by Appropriation A-17331. The Elkhorn River, which drains 7,000 square miles $\left(\mathrm{mi}^{2}\right)$, flows eastsoutheast through the Sand Hills in north-central Nebraska and the glaciated rolling hills of northeast Nebraska to its confluence with the Platte River about 20 miles downstream from Fremont, Nebraska (fig. 1). Logan Creek, a principal tributary of the Elkhorn River that drains much of the glaciated area of northeast Nebraska, enters the Elkhorn River north of Fremont. The flow regimes of the eastern and western parts of the Elkhorn River basin contrast because of the differences in the sediment composition and the increasing gradient of precipitation from west to east (Bentall and others, 1971). Streamflows in the Sand Hills or western part, which is characterized by extremely permeable sediments, are derived primarily from ground water. In contrast, streamflows in the eastern part, where loess-capped glacial deposits generally are less permeable, are more variable and responsive to precipitation events.

Surface-water use in the Elkhorn River basin includes irrigation, livestock, and recreational supplies. As of January 30, 2005, 362 surface-water appropriations with application dates later than 1970 were listed in the NDNR database (Nebraska Department of Natural Resources, 2005). These appropriations together are listed as irrigating about 29,000 acres in the Elkhorn River basin; however, the database does not include the number of appropriations or acreages adjudicated during this same period.

Salt Creek, whose confluence with the Platte River is about $7 \mathrm{mi}$ downstream from the confluence of the Elkhorn and Platte Rivers, flows north to northeast through southeast Nebraska. Its 1,650-mi ${ }^{2}$ basin generally slopes from southwest to northeast. Wahoo Creek, a principal tributary of Salt Creek, 


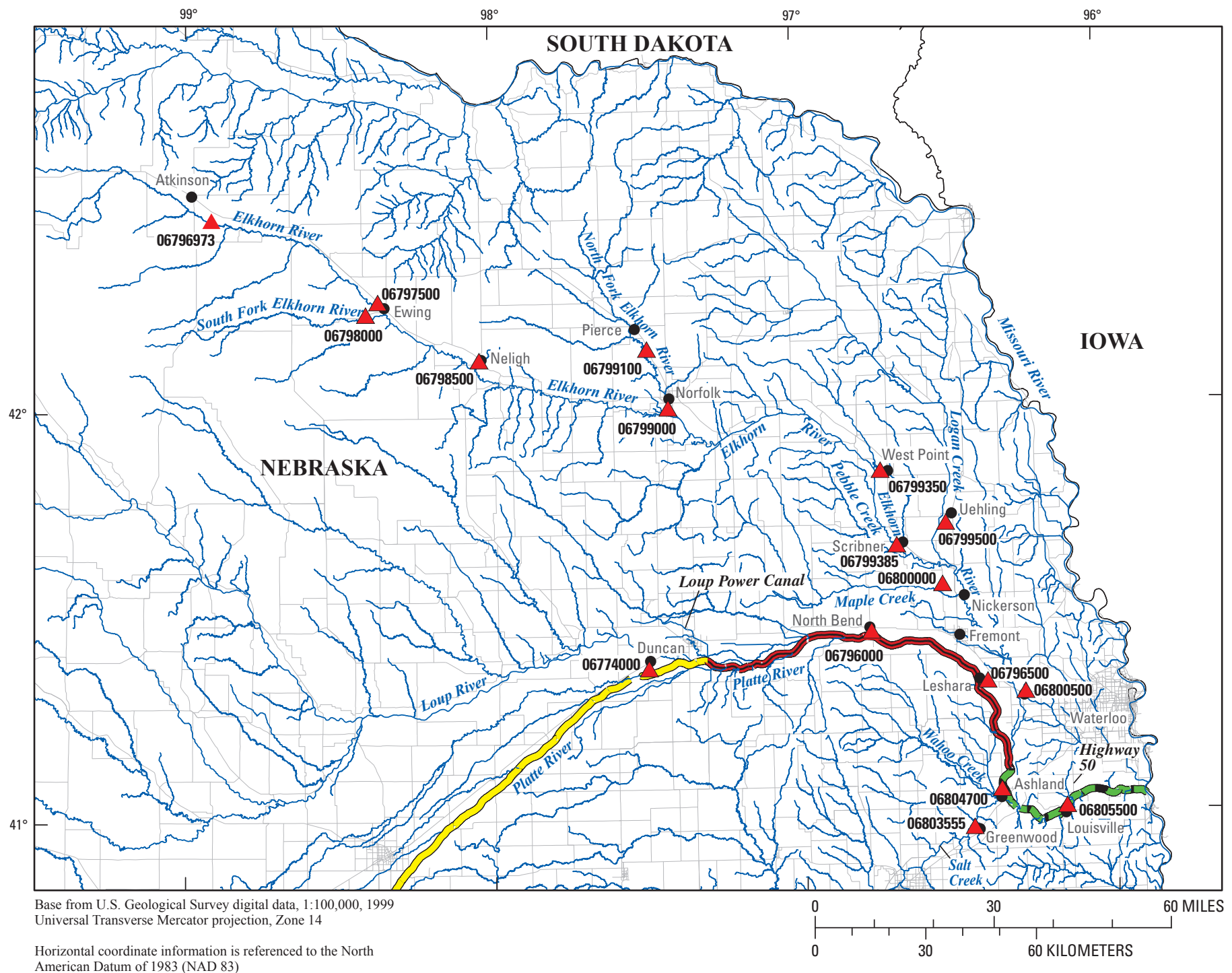

Horizontal coordinate information is referenced to the North American Datum of 1983 (NAD 83)

\section{EXPLANATION}

06803555 Streamflow-gaging station and number

Platte River Appropriation Reaches

Reach defined by Appropriation A-17329

Reach defined by Appropriation A-17330

Reach defined by Appropriation A-17331

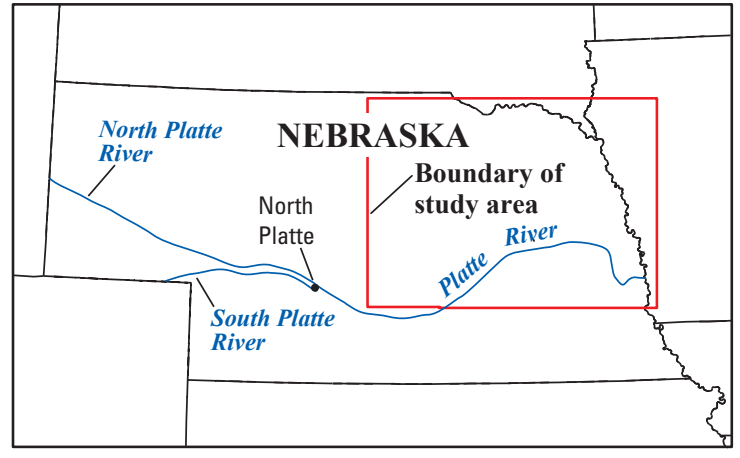

Index map

Figure 1. Study area and streamflow-gaging stations in the Elkhorn River, Salt Creek, and Platte River basins, Nebraska. 
enters Salt Creek near Ashland, or upstream from the confluence with the Platte River.

The Platte River is a braided stream that begins at the confluence of the North Platte and South Platte Rivers near North Platte, Nebraska, and has a drainage area of about $71,000 \mathrm{mi}^{2}$ at the USGS Platte River at Louisville, Nebraska, streamflow-gaging station (06805500). The recorded extremes for discharge at the Louisville streamflow-gaging station (for brevity, when referring to a Platte River streamflow-gaging station, hereinafter, only the geographic location will be used) range from 124,000 cubic feet per second $\left(\mathrm{ft}^{3} / \mathrm{s}\right)$ on March 30, 1960 , to $131 \mathrm{ft}^{3} / \mathrm{s}$ on September 3, 1976. From 1953 through 2005 , the mean annual discharge was $6,966 \mathrm{ft}^{3} / \mathrm{s}$ (U.S. Geological Survey, 2006).

\section{Background}

Trends in streamflow have been studied extensively on large scales within the United States (Lins and Slack, 1999; McCabe and Wolock, 2002; Milly, 2005). Lins and Slack (1999) detected positive trends in the annual minimum and median streamflow for 395 climate-sensitive streamflowgaging sites within the conterminous United States from 1944 through 1993 using the non-parametric Mann-Kendall test (Helsel and Hirsch, 1992). McCabe and Wolock (2002) used Kendall's Tau test (herein referred to as Kendall's Tau) to determine trends in minimum and median daily streamflow at sites within the conterminous United States. McCabe and Wolock found that the number of sites with positive trends was dependent on the time period analyzed. McCabe and Wolock stated that although monotonic trend tests such as Kendall's Tau cannot distinguish a gradual change from an abrupt change in a single data sequence, the temporal pattern of Kendall's Tau for many time periods supported the hypothesis of an abrupt step change in streamflow around 1970 being the dominant feature of temporal changes during the 1941-99 time period. McCabe and Wolock concluded that annual streamflows showed a "step" increase around 1970 rather than a gradual trend, and noted that these increases coincided with increased precipitation in the eastern United States as indicated by Karl and Knight (1998). Milly (2005) investigated trends in the water budget within the Mississippi River basin since 1949 and detected positive trends in precipitation that coincided with positive trends in runoff, but the trends in runoff also may have been emphasized at downstream sites by declines in the rate of filling of surface-water reservoirs constructed in the 1950s and 60s.

In Nebraska, instream flows for fish and wildlife purposes have been legally recognized as a beneficial use following the passage of legislation since 1984 (Aiken, 1989). Chapter 46-2 of the Nebraska State Statutes contains many of the provisions for acquiring an instream-flow appropriation. Instream-flow appropriations may be obtained by the NGPC or a Natural Resources District (NRD). The NGPC, NRD, or other state agencies must conduct detailed studies to quantify the amount of flow requested for a specific stream reach prior to requesting approval by NDNR (Aiken, 1989; Nebraska Revised State Statutes Section 46-2, 109). The director of NDNR may approve instream appropriation applications only after determining that: (1) unappropriated water is available for appropriation at a level that the species of interest can tolerate; (2) the appropriation is necessary to preserve the instream uses for which the appropriation has been requested; (3) the appropriation will not interfere with senior surface-water appropriations; (4) the rate and timing of flows are those required to maintain the instream use for which the appropriation has been requested; and (5) the instream flow is of public interest (Aiken, 1989; Nebraska Revised State Statutes Section 46-2, 115). Instream-flow appropriations are administered in the same manner as other appropriations with the exception that releases for reservoirs with senior water rights are not required to provide sufficient water for instream-flow appropriations (Nebraska Revised State Statutes Section 46-2, 119).

\section{Overview of Methods to Estimate Instream-Flow Requirements}

Habitat model simulations are commonly used to determine instream-flow requirements. Examples include the PHABSIM and modules of various hydraulic models. The PHABSIM is a habitat-simulation model developed using principles contained within the Instream Flow Incremental Methodology (IFIM) (Bovee, 1982; Bovee and others, 1998). Similar habitat models exist; however, those models were not used to determine instream-flow requirements for Appropriation A-17331 (Nebraska Department of Natural Resources,

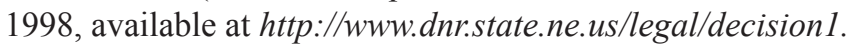
$\mathrm{htm}$ accessed January 30, 2005). Because PHABSIM was used to estimate instream-flow requirements for Appropriation A-17331 (Nebraska Department of Natural Resources, 1998), discussion of habitat models in this report primarily will focus on PHABSIM.

The PHABSIM and similar models use hydraulic principles to simulate water depths and velocities that are based on cross-section measurements made along the stream reach. Inputs for PHABSIM include channel geometry, substrate classification, vegetative cover indexes, and habitat suitability curves. Velocities and depths generated by the hydraulic part of the model are combined with cover and substrate information and habitat suitability criteria. These data were used to produce quality and quantity tables of usable habitat area for selected species at designated streamflows. In PHABSIM, criteria for favorable habitat are expressed as suitability index curves. A decimal scale ranging from 0 (entirely unsuitable) to 1 (completely suitable) is used to relate these index curves to physical measures such as depth, velocity, substrate, and cover to score microhabitat suitability. Experts possessing knowledge of favorable habitat conditions for species in the studied reaches may be called upon to collect data, perform habitat analyses, and use professional judgment to determine suitabil- 
ity index curves. More than one suitability index curve may exist for a particular reach; suitability index curves may be developed for multiple combinations of species, life stage, and season. The PHABSIM uses each suitability index curve for a particular reach to generate a series of relations of discharge to habitat areas. Relations of discharge to habitat area (habitat area also is called weighted usable area or WUA) are developed for each suitability index and then reported as square feet of usable habitat per 1,000 feet of stream length for a given discharge. The WUA can be combined for all considered species by first expressing the usable habitat as the percentage of optimum habitat $(\mathrm{POH})$ to eliminate bias in favor of species with large WUAs. The POH is the quotient of WUA divided by the maximum WUA simulated for each species or life stage. Where each species and life stage is given equal importance, $\mathrm{POH}$ values for each discharge can be averaged to determine a combined $\mathrm{POH}$. Alternatively, a weightedaveraging scheme can be used to place greater emphasis on species assigned a greater importance. The flow with the greatest combined $\mathrm{POH}$ can be selected as the single recommended flow for a particular reach; however, the greatest combined $\mathrm{POH}$ may occur at an unrealistic or low-frequency discharge, and other criteria for selecting a recommended flow may be required to provide a solution practical for stakeholders involved in implementing the recommended flow.

The Tennant standard-setting method (Tennant, 1976), sometimes called the Montana method, is another widely used method for estimating instream-flow requirements (table 1). Flows are computed as fixed percentages of mean annual streamflow for general regimes. The method requires streamflow data from a streamflow-gaging station on or near the stream reach being investigated. The Tennant method relies on the assumption that optimum habitat conditions occur near the mean annual streamflow.

A wide variety of additional methods and habitat modeling techniques exist for estimating habitat availability and for quantifying required instream flows. Methods developed by Poff and others (1997), range of variability approaches (Richter and others, 1997), and the Hydroecological Integrity Assessment Process (HIP) software tools (Henriksen and others, 2006) utilize the components of a flow regime such as magnitudes, frequencies, durations, timing, and rate of change to correlate with biological data to determine flows suitable for maintaining fish and wildlife habitat. The MesoHABSIM habitat model integrates the use of flow regime characteristics with other hydrological models to define community-specific thresholds (Annear and others, 2004). The R2CROSS and Wetted-Perimeter methods (Espegren, 1996) use channel geometry to determine flow-regime characteristics. Computer Aided Simulation Model for Instream Flow Requirements (CASIMIR) is another modular simulation model (Jorde and others, 2001).

\section{Overview of Appropriation A-17331}

For the purpose of protecting habitat for fish and wildlife on the Platte River in central and eastern Nebraska, on November 30, 1993, the NGPC filed five applications for water appropriations with the NDNR among which was the application for Appropriation A-17331. The initial application requested 4,000 $\mathrm{ft}^{3} / \mathrm{s}$ (September 16 through January 31), 5,800 ft $3 / \mathrm{s}$ (February 1 through June 15), and 4,000 $\mathrm{ft}^{3} / \mathrm{s}$ (June 16 through September 15) (R. Wozniak, Lower Elkhorn Natural Resources District, written commun., 2007). Efforts to estimate instream-flow requirements using PHABSIM and other estimation methodologies resulted in the revised flow-magnitude requests for the three applications listed in table 2 and described below (Nebraska Department of Natural Resources, 1998).

PHABSIM was implemented by Twelve Nine, Inc. (1990), Hardy and Associates (1992), and Schainost and others (1993). Inputs to PHABSIM included suitability index curves developed by Peters and others (1989) and Chadwick and Associates (written commun., 1994) for 12 fish species of the lower Platte River. Hydraulic data, including surveyed channel elevations, water depth and velocity measurements, and habitat data, including cover and substrate size distribution, were collected on the Platte River in 1985, 1987, and 1988 by an interagency team composed of federal, state, university, and agency personnel.

Table 1. Tennant standard-setting method for estimating instream-flow requirements (Tennant, 1976).

\begin{tabular}{lcc}
\hline \multirow{2}{*}{ Flow description } & \multicolumn{2}{c}{ Instream flow } \\
\cline { 2 - 3 } & $\begin{array}{c}\text { October-March (Winter), as a per- } \\
\text { centage of mean annual streamflow }\end{array}$ & $\begin{array}{c}\text { April-September (Summer), as a per- } \\
\text { centage of mean annual streamflow }\end{array}$ \\
\hline Flushing or maximum & 200 & 200 \\
Optimum range & $60-100$ & $60-100$ \\
Outstanding & 40 & 60 \\
Excellent & 30 & 50 \\
Good & 20 & 40 \\
Fair or degrading & 10 & 30 \\
Poor or minimum & 10 & 10 \\
Severe degradation & $0-10$ & $0-10$ \\
\hline
\end{tabular}


Trends in Streamflow Characteristics of Selected Sites in the Elkhorn River, Salt Creek, and Lower Platte River Basins

Table 2. Summary of Nebraska Game and Parks Commission applications for instream-flow appropriations, Platte River, Nebraska (Nebraska Department of Natural Resources, 1998, available at $h$ ttp://www.dnr.state.ne.us/legal/decision1.htm accessed January 30, 2005).

\begin{tabular}{lcccc}
\hline $\begin{array}{c}\text { Appropriation } \\
\text { number }\end{array}$ & Purpose & Platte River reach & Time & $\begin{array}{c}\text { Flow requested, } \\
\text { in cubic feet per } \\
\text { second }\end{array}$ \\
\hline A-17329 & $\begin{array}{r}\text { Maintain fish } \\
\text { community }\end{array}$ & $\begin{array}{c}\text { Kearney Canal diversion } \\
\text { downstream to Loup } \\
\text { Power Canal return }\end{array}$ & All Year & 1,000 \\
A-17330 & $\begin{array}{c}\text { Maintain fish } \\
\text { community }\end{array}$ & $\begin{array}{c}\text { Loup Power Canal return to } \\
\text { confluence with Elkhorn } \\
\text { River }\end{array}$ & All Year & 1,800 \\
A-17331 & Maintain fish & Confluence with Elkhorn \\
& community & River to confluence with & All Year & 3,700 \\
& & Missouri River & \\
\hline
\end{tabular}

For instream-flow requests for the Platte River, the potential bias in favor of species with large WUA values was removed by converting each WUA-discharge relation to a POH-discharge relation per species or life stage. A simple average giving equal importance to all fish species and life stages was used to develop an overall $\mathrm{POH}$ value per discharge (Zuerlein and others, 2001). Analysis of the PHABSIM results for all fish species indicated that habitat availability in the lower Platte River rapidly declines as flows decrease below $2,000 \mathrm{ft}^{3} / \mathrm{s}$. Based on PHABSIM analysis, the NGPC requested instream-flow appropriations of $1,800 \mathrm{ft}^{3} / \mathrm{s}$ and $3,700 \mathrm{ft}^{3} / \mathrm{s}$ for the Platte River reaches from the Loup River to the Elkhorn River (A-17330) and from the Elkhorn River to the mouth of the Platte (A-17331), respectively (Zuerlein and others, 2001). Criticism during the application review process concerned use of the fixed-bed assumption in the PHABSIM model for the sandy, shifting bed of the Platte River, and whether or not an adequate number of study sites were included in the analysis (Nebraska Department of Natural Resources, 1998). However, proponents of PHABSIM argued that the relatively long reach of the Platte River was in dynamic equilibrium, and losses of microhabitat because of sediment erosion in some parts of the reach were offset by sediment deposition in other parts of the reach, and that study sites included in the analysis were representative of the river (Milhous and others, 1984; Nebraska Department of Natural Resources, 1998). Further evaluations of PHABSIM output failed to provide conclusive evidence validating or invalidating its flow recommendations (Nebraska Department of Natural Resources, 1998). Of the five applications filed by NGPC for the purpose of protecting fish and wildlife habitat in the central and eastern Platte River, four were approved for instream water appropriations by the NDNR. The following sections describe the criteria, process, and approval status of the five NGPC appropriation applications with emphasis on A-17329, A-17330, and A-17331.

Appropriation A-17329 - Johnson Power Plant return to Loup Power Canal return. Water-temperature criteria were used for selecting recommended flows on the Platte River for Appropriation A-17329. NGPC was concerned that low flows and shallow depths in the central Platte River during summer months might result in increased water temperatures and reduced concentrations of dissolved oxygen, which stress fish and potentially result in fish kills (Nebraska Department of Natural Resources, 1998). NGPC requested a 1,000 ft's appropriation because additional analysis using the Tennant (1976) method, a U.S. Fish and Wildlife (USFWS) fish guild PHABSIM study, a habitat richness analysis, and a water temperature compared to discharge analysis indicated that 650 $\mathrm{ft}^{3} / \mathrm{s}$ would be insufficient to protect the resources (Zuerlein and others, 2001).

Appropriation A-17330 - Loup Power Canal return to confluence with Elkhorn River; and Appropriation A-17331Confluence with Elkhorn River to confluence with Missouri River. Both appropriations (table 2) were requested for protection of fish communities through maintenance of streamflow levels. Flow recommendations were estimated through analysis of PHABSIM simulations (Milhous and others, 1984). Based on those results, NGPC requested instream-flow appropriations of $1,800 \mathrm{ft}^{3} / \mathrm{s}$ for the reach from the Loup River to the Elkhorn River, and 3,700 $\mathrm{ft}^{3} / \mathrm{s}$ for the reach from the Elkhorn River to the mouth of the Platte (Zuerlein and others, 2001).

In a June 26, 1998, decision issued by NDNR, the five NGPC applications for instream appropriations were approved, modified, or denied. Appropriation A-17329 was approved such that the NGPC appropriation, combined with the senior Central Platte Natural Resources District (CPNRD) instream appropriation, would equal $1,000 \mathrm{ft}^{3} / \mathrm{s}$ in June and July (table 3). During August, the combined appropriated flow would equal $800 \mathrm{ft}^{3} / \mathrm{s}$ at Odessa, Nebraska, and $900 \mathrm{ft}^{3} / \mathrm{s}$ near Duncan, Nebraska. The August flow values were selected such that appropriated flow would achieve a 20-percent exceedance threshold seasonally (appropriated flow is exceeded more than 20 percent of the time for the given time period). Appropriation A-17330 was approved by NDNR as requested (table 3). Appropriation A-17331 was modified to account for the senior Metropolitan Utilities District (MUD) water right of $500 \mathrm{ft}^{3} / \mathrm{s}$ and to meet the 20-percent threshold flow-duration value. 
Table 3. Platte River instream flows appropriated by the Nebraska Department of Natural Resources to the Nebraska Game and Parks Commission on June 26, 1998 (Zuerlein and others, 2001).

[ $\mathrm{ft}^{3} / \mathrm{s}$, cubic feet per second; -, appropriation not applicable; CPNRD, Central Platte Natural Resources District; MUD, Metropolitan Utilities District ]

\begin{tabular}{|c|c|c|c|c|c|c|c|}
\hline \multirow[b]{3}{*}{$\begin{array}{l}\text { Appropriation num- } \\
\text { ber and approved } \\
\text { flow (except for } \\
\text { specified seasons) }\end{array}$} & \multirow[b]{3}{*}{ Benefit } & \multirow[b]{3}{*}{ River segment } & \multirow[b]{3}{*}{ Season } & \multicolumn{4}{|c|}{ Seasonal appropriated flow, in $\mathrm{ft}^{3} / \mathrm{s}$} \\
\hline & & & & \multicolumn{2}{|c|}{ Central Platte River reach } & \multicolumn{2}{|c|}{ Lower Platte River reach } \\
\hline & & & & $\begin{array}{l}\text { Odessa and } \\
\text { Grand Island } \\
\text { streamflow- } \\
\text { gaging sta- } \\
\text { tions }\end{array}$ & $\begin{array}{l}\text { Duncan } \\
\text { stream- } \\
\text { flow- } \\
\text { gaging } \\
\text { station }\end{array}$ & $\begin{array}{l}\text { North Bend } \\
\text { streamflow- } \\
\text { gaging } \\
\text { station }\end{array}$ & $\begin{array}{c}\text { Louisville } \\
\text { streamflow- } \\
\text { gaging station }\end{array}$ \\
\hline \multirow[t]{5}{*}{ A-17329 } & \multirow{5}{*}{$\begin{array}{l}\text { Fish commu- } \\
\text { nity }\end{array}$} & \multirow{5}{*}{$\begin{array}{l}\text { Kearney Canal } \\
\text { diversion } \\
\text { downstream } \\
\text { to Loup } \\
\text { Power Canal } \\
\text { return }\end{array}$} & Sept.-May & 1,000 & 1,000 & - & - \\
\hline & & & June 1-June 23 & 1500 & 1500 & - & - \\
\hline & & & June 24-July 31 & ${ }^{1} 400$ & ${ }^{1} 400$ & - & - \\
\hline & & & Aug. 1-Aug. 22 & ${ }^{2} 200$ & ${ }^{3} 300$ & - & - \\
\hline & & & Aug. 23-Aug. 31 & 2300 & ${ }^{3} 400$ & - & - \\
\hline A-17330 & $\begin{array}{l}\text { Fish commu- } \\
\text { nity }\end{array}$ & $\begin{array}{l}\text { Loup Power } \\
\text { Canal return } \\
\text { to confluence } \\
\text { with Elkhorn } \\
\text { River }\end{array}$ & Entire year & - & - & 1,800 & - \\
\hline \multirow[t]{5}{*}{ A-17331 } & \multirow{5}{*}{$\begin{array}{l}\text { Fish commu- } \\
\text { nity }\end{array}$} & \multirow{5}{*}{$\begin{array}{c}\text { Elkhorn River } \\
\text { downstream } \\
\text { to mouth of } \\
\text { Platte River }\end{array}$} & January & - & - & - & ${ }^{4} 3,100$ \\
\hline & & & Feb.-July & - & - & - & ${ }^{4} 3,700$ \\
\hline & & & August & - & - & - & ${ }^{4} 3,500$ \\
\hline & & & September & - & - & - & 43,200 \\
\hline & & & Oct.-Dec. & - & - & - & 43,700 \\
\hline \multicolumn{8}{|c|}{${ }^{1}$ Combined appropriated flows with CPNRD equal $1,000 \mathrm{ft}^{3} / \mathrm{s}$. } \\
\hline \multicolumn{8}{|c|}{${ }^{2}$ Combined appropriated flows with CPNRD equal 800 ft³/s. } \\
\hline \multicolumn{8}{|c|}{${ }^{3}$ Combined appropriated flows with CPNRD equal $900 \mathrm{ft}^{3} / \mathrm{s}$. } \\
\hline
\end{tabular}

\section{Methods of Study}

\section{Streamflow Characteristics}

To evaluate streamflow characteristics, statistical summaries were computed for 17 streamflow-gaging stations in the Elkhorn River, Platte River, and Salt Creek drainage basins (table 4). Periods of streamflow-gaging station operation ranged from 1896 to present (September 30, 2004) in the Platte River and Elkhorn River basins and from the 1950s to present in the Salt Creek basin. Periods of daily streamflow records used for statistical calculations and trends analysis ranged from 1928 to 2004 and included data from the 2005 water year (WY) if published records were available at the time of analysis. A water year begins October 1 of each calendar year and ends September 30 of the following calendar year.

Flow duration tables describe the percentage of time specified flows were exceeded during a given period, and nonexceedance duration hydrographs graphically describe flow percentiles for each day of the calendar year based on histori- cal daily mean flows (for example, table 5 and fig. 2). Flow duration tables provide broad summaries of streamflow data for a given period and are particularly useful for comparing different sites during similar time periods; however, duration tables do not preserve the chronological characteristics of flow. Duration hydrographs represent the flow percentiles for each calendar day in a compact plot, and are useful for evaluating seasonal variations in streamflow for the period of record. Flow duration tables and duration hydrographs provide useful tools for interpreting flows during the period included in the tables and plots but inadequately represent the sequential occurrences of low-flow events from year to year.

Daily mean streamflows were used to identify extreme low-flow periods for specific numbers of consecutive days, or n-day low flows for each year. Low-flow values most often are computed as the annual minimum mean flows for $n$ consecutive days during a climatic year (April to March). For example, the annual 7-day low flow describes the lowest mean flow among all periods of 7 consecutive days during a year (fig. 3). Climatic years, as used in these analyses, typically are used in low-flow analyses because low flows in much of the United States often occur in late summer and early fall, and the designation of the flow period by WY (October-September) may 
Table 4. Periods of operation of U.S. Geological Survey and Nebraska Department of Natural Resources streamflowgaging stations in the Elkhorn River, Salt Creek, and Platte River basins, Nebraska.

\begin{tabular}{cllc}
\hline $\begin{array}{c}\text { Streamflow-gaging } \\
\text { station number (fig. 1) }\end{array}$ & \multicolumn{1}{c}{ Station name } & $\begin{array}{c}\text { Date of station estab- } \\
\text { lishment }^{1}\end{array}$ & ${\text { Years of record }{ }^{2}}^{-1}$ \\
\hline 06774000 & Platte River near Duncan, Nebraska & $04 / 01 / 1941$ & 63 \\
06796000 & Platte River at North Bend, Nebraska & $04 / 01 / 1949$ & 55 \\
06796500 & Platte River near Leshara, Nebraska & $06 / 29 / 1994$ & 10 \\
06796973 & Elkhorn River near Atkinson, Nebraska & $10 / 01 / 1982$ & 22 \\
06797500 & Elkhorn River at Ewing, Nebraska & $08 / 01 / 1947$ & 57 \\
06798000 & South Fork Elkhorn River at Ewing, Nebraska & $08 / 01 / 1947$ & 57 \\
06798500 & Elkhorn River at Neligh, Nebraska & $10 / 01 / 1930$ & 75 \\
06799000 & Elkhorn River at Norfolk, Nebraska & $08 / 01 / 1896$ & 63 \\
06799100 & North Fork Elkhorn River near Pierce, Nebraska & $08 / 01 / 1960$ & 44 \\
06799350 & Elkhorn River at West Point, Nebraska & $10 / 01 / 1972$ & 32 \\
06799385 & Pebble Creek at Scribner, Nebraska & $10 / 01 / 1978$ & 27 \\
06799500 & Logan Creek near Uehling, Nebraska & $04 / 01 / 1941$ & 63 \\
06800000 & Maple Creek near Nickerson, Nebraska & $10 / 01 / 1951$ & 53 \\
06800500 & Elkhorn River at Waterloo, Nebraska & $09 / 01 / 1928$ & 76 \\
06803555 & Salt Creek at Greenwood, Nebraska & $11 / 01 / 1951$ & 53 \\
06804700 & Wahoo Creek at Ashland, Nebraska & $08 / 31 / 1990$ & 14 \\
06805500 & Platte River at Louisville, Nebraska & $06 / 01 / 1953$ & 51
\end{tabular}

${ }^{1}$ All streamflow-gaging stations were operational in 2004.

${ }^{2}$ Years of record may not match years computed from period of operation because of missing annual mean streamflow values or periods of temporary inactivity.

separate one low-flow period into 2 water years. Low-flow values were determined for the 17 selected sites in the study area for consecutive durations of 1-, 3-, 7-, 14-, 30-, 60-, and 183-days for the available period of record through September 30,2004 or later if published data were available at the time of analysis.

Low-flow probability values were computed for consecutive low-flow periods using log-Pearson type III analysis (Interagency Advisory Committee on Water Data, 1982). Lowflow probability values provide an estimate of the probability of occurrence for a particular minimum $n$-day flow during any single year. For instance, 7-day low-flow probability values indicate that, for the 5-percent annual chance, the indicated minimum average flow for $n$-days of the climatic year is likely to occur 5 times, on average, during a given 100-year period. The recurrence interval (equation 1 ) is defined as the inverse of the percent annual chance $(P)$, which in the previous example is equal to 20 years.

$$
\text { Reference interval }=\frac{100 \text { percent }}{P}
$$

Generally, the measured streamflow of the Platte River at Louisville, Nebraska, is the sum of streamflows measured at streamflow-gaging stations on the Elkhorn River at Waterloo, Salt Creek at Greenwood, Wahoo Creek at Ashland, and Platte River at Ashland, plus any unmeasured inflows minus any outflows between these streamflow-gaging stations and the Louisville streamflow-gaging station. Annual streamflow records for the streamflow-gaging stations at Elkhorn River at Waterloo, Salt Creek at Greenwood, and Wahoo Creek at Ashland were compared to streamflows measured at the Platte River at Louisville streamflow-gaging station to estimate the effects of the Salt Creek and Elkhorn River Basins on the flows of the Platte River at Louisville.

\section{Streamflow Trends}

Kendall's Tau test is widely used to detect trends in timeseries data (Helsel and Hirsch, 1992). Kendall's Tau was used to detect temporal trends in selected streamflow statistics at 17 sites in the study area (all sites are located in Nebraska). The Tau statistic is a measure of the level of upward or downward change in a data set; a Tau value of +1 indicates each element in the series is greater than the previous element in the series, and -1 indicates each element in the series is less than the previous element in the series. The statistic represented by $p$ is used to quantify the probability that the available evidence to conclude for a trend slope different from zero could have arisen when there actually was no temporal trend. A significance level of 0.95 ( $p$-value $<0.05)$ was used to declare the presence of a trend. Kendall's Tau was performed for annual mean flows, monthly mean flows for each month of the year, and annual low flows (1-, 3-, 7-, 14-, 30-, 60-, and 183-day) 
for the period of record at each site. The streamflow values were not adjusted to remove the effect of precipitation variation.

Linear regression analyses were used to estimate the trend slope in time-series data for sites with significant temporal trends. A best-fit line (least-squares regression) was determined for the streamflow data, and the trend slope was computed using SWSTAT (Lumb and others, 1990). This slope represents the median magnitude of the annual increase or decrease in the respective streamflow statistic over the period analyzed.

\section{Historical Streamflow Characteristics}

In order to illustrate annual and seasonal variations in streamflows, flow duration tables, duration hydrographs, and low-flow probability values are presented for each selected streamflow-gaging station in Appendix 1.

At the streamflow-gaging station at Louisville, Nebraska, flows generally were greatest in the winter and spring but declined through the summer. During parts of October through December, flow criteria required for Appropriation A-17331 generally fall between the 10th and 50th non-exceedance percentiles (fig. 2). In January, flow criteria required for Appropriation A-17331 are near the 30th percentile. During February through June, flow criteria required for Appropriation A-17331 generally are less than the 30th percentile, but fall within the range from less than the minimum recorded streamflow to the 10th percentile for many calendar days. During July through parts of October, flow criteria required for Appropriation A-17331 generally fall between the 30th and 70th percentiles.

The duration table (table 5) indicates that criteria required for Appropriation A-17331 were exceeded from 65 to 70 percent of the time at the Platte River at Louisville streamflowgaging station. A comparison of the duration hydrographs for the streamflow-gaging stations at Platte River near Duncan and Platte River at Louisville indicated that streamflow $1 \mathrm{ft}^{3} / \mathrm{s}$ or less occurred more frequently at the Platte River near Duncan streamflow-gaging station during its period of available streamflow data (1928 to 2004) than at the Platte River at Louisville streamflow-gaging station during its period of available streamflow data (1953 to 2004). The duration hydrograph for Platte River near Duncan streamflow-gaging station indicates that flows not exceeding $1 \mathrm{ft}^{3} / \mathrm{s}$ occur within the minimum to 10th percentile range during parts of November, December, January, June, and July; within the 10th to 20th percentile range during parts of October, July, August, and September; and within the 20th to 30th percentile range during parts of August and September (Appendix 1).

The flows recorded at the Salt Creek at Greenwood streamflow-gaging station and the Elkhorn River at Waterloo streamflow-gaging station contribute part of the flow recorded at the Platte River at Louisville streamflow-gaging station. The remainder of the flow recorded at the Platte River at Louisville 


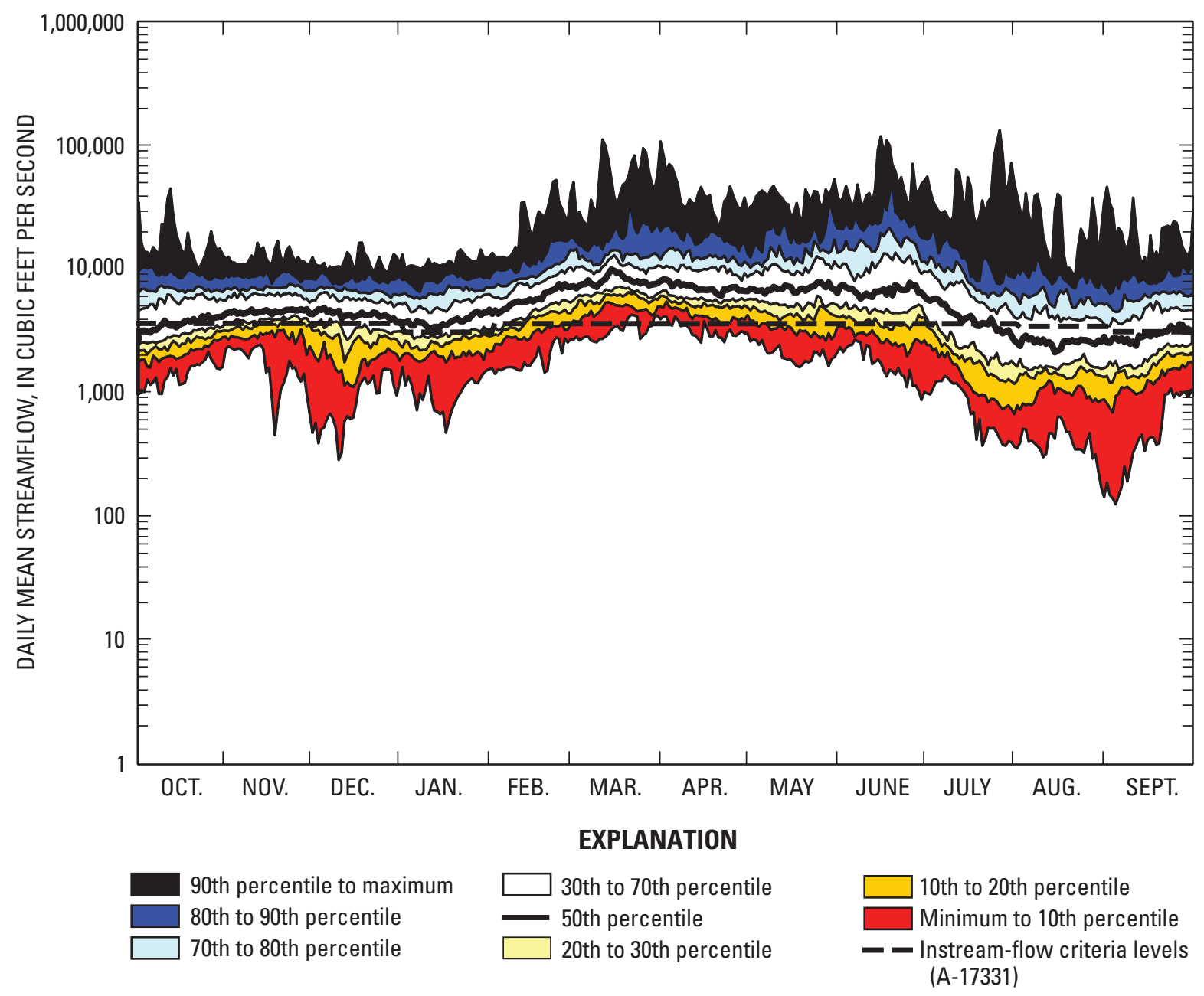

Figure 2. Non-exceedance duration hydrograph for Platte River at Louisville, Nebraska, for 1953-2004.

streamflow-gaging station can be attributed to the Platte River upstream from the mouth of the Elkhorn River, Wahoo Creek, minor inflows and outflows between the mouth of the Elkhorn River and the Platte River at Louisville streamflow-gaging station, and minor inflows and outflows between the mouth of the Elkhorn River and the Elkhorn River at Waterloo streamflow-gaging station. During WY 1954 through 2004, about 21 percent of the annual mean flows recorded at the Platte River at Louisville streamflow-gaging station was measured in the Elkhorn River at Waterloo streamflow-gaging station (table 6). Annual flows of the Elkhorn River at Waterloo streamflow-gaging station ranged from about 12 to 30 percent of annual mean flows recorded at the Platte River at Louisville streamflow-gaging station with a standard deviation of about 4 percent. During this period, flow recorded at the Salt Creek at Greenwood streamflow-gaging station composed, on average, 5 percent of the annual flow recorded at the Platte River at Louisville streamflow-gaging station, with a range of about 2 to 10 percent and a standard deviation of about 2 percent. In the years prior to the application for Appropriation A-17331 (WY 1954 through 1992), 19 percent of the annual mean flows recorded at the Platte River at Louisville streamflow- gaging station was measured in the Elkhorn River at Waterloo streamflow-gaging station. In the years after the application for Appropriation A-17331 (WY 1993 through 2004), 25 percent of the annual mean flows measured at the Platte River at Louisville streamflow-gaging station was measured in the Elkhorn River at Waterloo streamflow-gaging station.

Results of Kendall's Tau for temporal trends in annual mean flows for the full period of record are presented in table 7 (Appendix 2). Positive trends were found for annual mean flows at eight sites: Platte River near Duncan; Platte River at North Bend; Elkhorn River at Neligh; Logan Creek near Uehling; Maple Creek near Nickerson; Elkhorn River at Waterloo; Salt Creek at Greenwood; and Platte River at Louisville. A negative trend was found for annual mean flows at the Platte River near Leshara streamflow-gaging station, though this temporal trend may be strongly affected by the short period of available data for this site. Trends in annual mean flows were not significant at these sites: Elkhorn River near Atkinson; Elkhorn River at Ewing; South Fork Elkhorn River at Ewing; Elkhorn River at Norfolk; North Fork Elkhorn River near Pierce; Elkhorn River at West Point; Pebble Creek at Scribner; and Wahoo Creek at Ashland. 


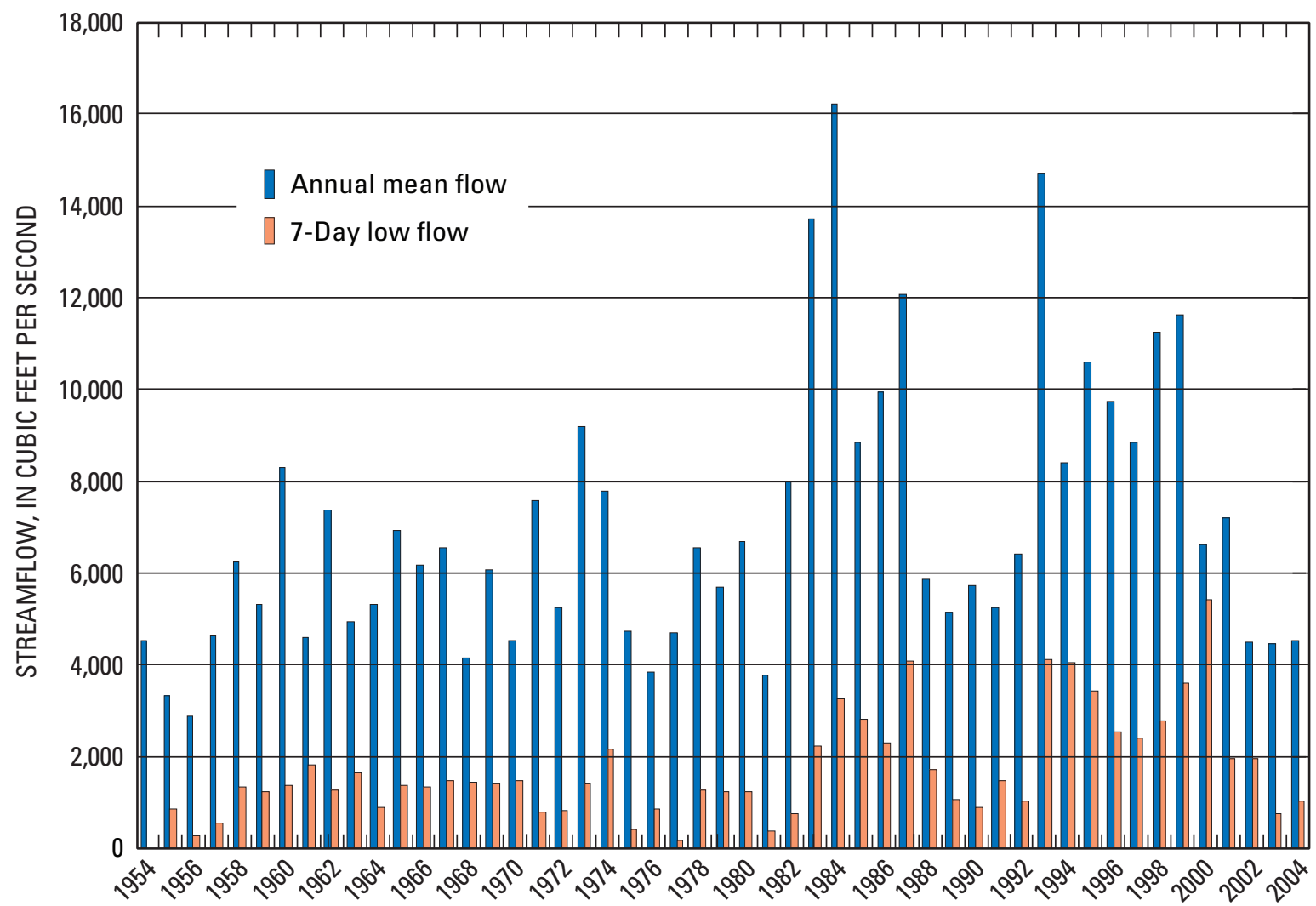

Figure 3. Annual mean streamflow and 7-day low flow of Platte River at Louisville, Nebraska, for 1954-2004.

Sites that showed positive trends for annual minimum 1-day low flows included: Platte River near Duncan; South Fork Elkhorn River at Ewing; Elkhorn River at Neligh; Pebble Creek at Scribner; Logan Creek near Uehling; Maple Creek near Nickerson; Elkhorn River at Waterloo; Salt Creek at Greenwood; and Platte River at Louisville. There were no sites with negative trends in annual minimum 1-day low flows. Trends in annual minimum 1-day low flows were not significant at these sites: Platte River at North Bend; Elkhorn River near Atkinson; Elkhorn River at Ewing; Elkhorn River at Norfolk; North Fork Elkhorn River near Pierce; Elkhorn River at West Point; Platte River near Leshara; and Wahoo Creek at Ashland.

Sites that showed positive trends for annual minimum 7-day low flows included: Platte River near Duncan; South Fork Elkhorn River at Ewing; Elkhorn River at Neligh; Pebble Creek at Scribner; Logan Creek near Uehling; Maple Creek near Nickerson; Elkhorn River at Waterloo; Salt Creek at Greenwood; and Platte River at Louisville. There were no sites with negative trends in 7-day low flows. Trends in 7-day low flows were not significant at these sites: Platte River at North Bend; Elkhorn River near Atkinson; Elkhorn River at Ewing;

Table 6. Summary statistics for annual mean streamflow expressed as a percentage of the annual mean streamflow at the U.S. Geological Survey streamflow-gaging station on Platte River at Louisville, Nebraska (station 06805500), for water years 1954 through 2004.

\begin{tabular}{cccccc}
\hline & \multicolumn{2}{c}{$\begin{array}{c}\text { Streamflow statistic, as a percentage of the Platte River at Louisville } \\
\text { streamflow-gaging station mean annual flow }\end{array}$} \\
\cline { 2 - 6 } Source of streamflow & Mean & Median & Maximum & Minimum & $\begin{array}{c}\text { Standard } \\
\text { deviation }\end{array}$ \\
\hline $\begin{array}{c}\text { 06803555 Salt Creek at } \\
\text { Greenwood, Nebraska }\end{array}$ & 4.7 & 4.8 & 9.7 & 2.5 & 1.6 \\
$\begin{array}{c}\text { 06800500 Elkhorn River } \\
\text { at Waterloo, Nebraska }\end{array}$ & 20.5 & 20.6 & 30.5 & 11.9 & 4.3 \\
$\begin{array}{c}\text { Other sources including } \\
\text { the Platte River up- } \\
\text { stream from the mouth } \\
\text { of the Elkhorn River }\end{array}$ & 74.6 & 74.5 & 83.6 & 65.1 & 4.6 \\
\hline
\end{tabular}


Table 7. Median slope of significant low-flow trends at selected sites in the Elkhorn River, Salt Creek, and Platte River basins, Nebraska.

[Significant trends had $p$-values less than 0.05; I, insignificant trend; in cubic feet per second per year; POS, positive trend, slope not calculated]

\begin{tabular}{|c|c|c|c|c|c|c|c|c|c|c|c|c|c|c|c|c|c|c|c|c|c|}
\hline \multirow{2}{*}{\multicolumn{2}{|c|}{$\begin{array}{c}\text { U.S. Geological Survey } \\
\text { streamflow-gaging station } \\
\text { number and name }\end{array}$}} & \multirow{2}{*}{$\begin{array}{c}\text { Period } \\
\text { analyzed for } \\
\text { annual mean } \\
\text { flow (see } \\
\text { Appendix } 2 \\
\text { for period } \\
\text { analyzed for } \\
\text { other } \\
\text { streamflow } \\
\text { statistics) }\end{array}$} & \multicolumn{10}{|c|}{ Annual } & \multirow[b]{2}{*}{ 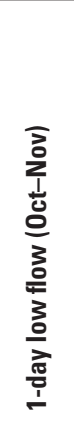 } & \multirow[b]{2}{*}{ 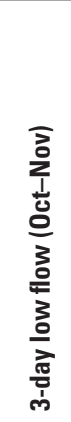 } & \multirow[b]{2}{*}{ 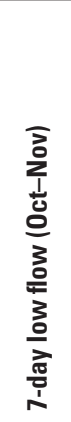 } & \multirow[b]{2}{*}{ 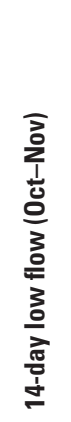 } & \multirow[b]{2}{*}{ 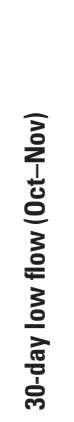 } & \multirow[b]{2}{*}{ 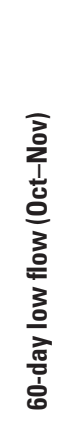 } & \multirow[b]{2}{*}{ 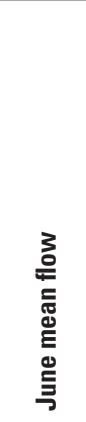 } & \multirow[b]{2}{*}{ 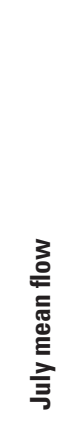 } & \multirow[b]{2}{*}{ 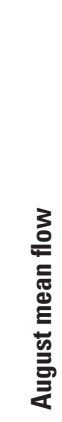 } \\
\hline & & & 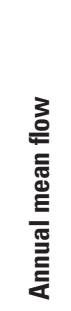 & $\begin{array}{l}3 \\
\frac{3}{0} \\
3 \\
\frac{3}{0} \\
\frac{\pi}{2} \\
\frac{1}{1} \\
-1\end{array}$ & 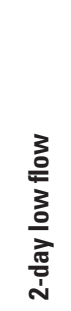 & 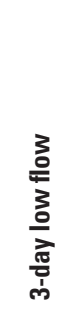 & 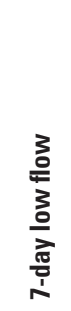 & 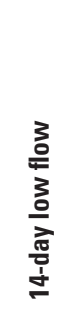 & 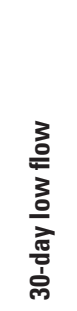 & 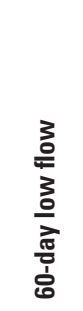 & $\begin{array}{l}\frac{3}{0} \\
\frac{0}{3} \\
\frac{3}{0} \\
\frac{\pi}{0} \\
\text { ò }\end{array}$ & 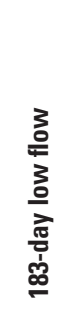 & & & & & & & & & \\
\hline 06774000 & $\begin{array}{l}\text { Platte River } \\
\text { near Duncan, } \\
\text { Nebr. }\end{array}$ & 1929-2003 & +13 & +1.1 & +1.2 & +1.3 & +1.6 & +2.5 & +4.8 & +7.5 & +11 & +12 & +8.2 & +8.7 & +10 & +12 & +13 & +14 & I & I & +21 \\
\hline 06796000 & $\begin{array}{l}\text { Platte River at } \\
\text { North Bend, } \\
\text { Nebr. }\end{array}$ & 1949-2003 & +26 & I & I & I & I & I & +14 & +19 & +22 & +23 & +18 & +24 & +23 & +25 & +27 & +30 & I & I & I \\
\hline 06796500 & $\begin{array}{l}\text { Platte River } \\
\text { near Leshara, } \\
\text { Nebr. }\end{array}$ & 1994-2004 & -510 & I & I & I & I & I & I & -380 & -450 & -430 & -280 & I & -330 & -350 & -390 & -450 & -1100 & -610 & -530 \\
\hline 06796973 & $\begin{array}{c}\text { Elkhorn River } \\
\text { near Atkin- } \\
\text { son, Nebr. }\end{array}$ & 1983-2003 & I & I & I & I & I & I & I & I & I & I & I & I & I & I & I & I & +1 & -3.5 & -1.3 \\
\hline 06797500 & $\begin{array}{c}\text { Elkhorn River at } \\
\text { Ewing, Nebr. }\end{array}$ & $1947-2003$ & I & I & I & I & I & I & I & I & I & I & I & I & I & I & I & I & I & I & I \\
\hline 06798000 & $\begin{array}{l}\text { South Fork } \\
\text { Elkhorn Riv } \\
\text { er at Ewing, } \\
\text { Nebr. }\end{array}$ & 1947-2003 & I & +.2 & +.2 & +.1 & +.1 & I & I & I & I & I & +.2 & +.1 & I & I & I & I & I & +.1 & I \\
\hline 06798500 & $\begin{array}{l}\text { Elkhorn River at } \\
\text { Neligh, Nebr. }\end{array}$ & 1931-2003 & +2.6 & +.5 & +.5 & +.5 & +.6 & I & I & +.6 & +.7 & +.8 & +2.2 & I & I & I & I & +.7 & +1.4 & +3.1 & I \\
\hline 06799000 & $\begin{array}{l}\text { Elkhorn River } \\
\text { at Norfolk, } \\
\text { Nebr. }\end{array}$ & 1896-2003 & I & I & I & I & I & I & I & I & I & I & I & I & I & I & I & I & I & I & I \\
\hline 06799100 & $\begin{array}{l}\text { North Fork Elk- } \\
\text { horn River } \\
\text { near Pierce, } \\
\text { Nebr. }\end{array}$ & $1960-2003$ & I & I & I & I & I & I & I & I & I & I & I & I & I & I & I & I & I & I & I \\
\hline 06799350 & $\begin{array}{l}\text { Elkhorn River at } \\
\text { West Point, } \\
\text { Nebr. }\end{array}$ & 1973-2003 & I & I & I & I & I & I & +7.2 & +9.1 & +9.8 & +9.7 & I & I & I & I & +8.3 & I & I & +35 & I \\
\hline
\end{tabular}


Table 7. Median slope of significant low-flow trends at selected sites in the Elkhorn River, Salt Creek, and Platte River basins, Nebraska.—Continued [Significant trends had $p$-values less than 0.05; I, insignificant trend; in cubic feet per second per year; POS, positive trend, slope not calculated]

\begin{tabular}{|c|c|c|c|c|c|c|c|c|c|c|c|c|c|c|c|c|c|c|c|c|c|}
\hline & & Derind & & & & & Ann & & & & & & & & & & & & & & \\
\hline $\begin{array}{r}\text { U.S. Ge } \\
\text { streamflc } \\
\text { num }\end{array}$ & $\begin{array}{l}\text { logical Survey } \\
N \text {-gaging station } \\
\text { er and name }\end{array}$ & $\begin{array}{c}\text { annual mean } \\
\text { flow (see } \\
\text { Appendix } 2 \\
\text { for period } \\
\text { analyzed for } \\
\text { other } \\
\text { streamflow } \\
\text { statistics) }\end{array}$ & 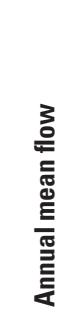 & $\begin{array}{l}\frac{3}{0} \\
\frac{0}{1} \\
\frac{3}{0} \\
\frac{0}{\pi} \\
\frac{\pi}{1}\end{array}$ & $\begin{array}{l}\frac{3}{0} \\
\frac{0}{3} \\
\frac{3}{0} \\
\frac{\pi}{i} \\
\text { ì }\end{array}$ & 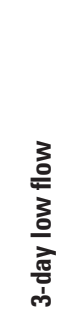 & $\begin{array}{l}3 \\
0 \\
\frac{3}{0} \\
\frac{3}{0} \\
\frac{0}{2} \\
\frac{\pi}{1}\end{array}$ & $\begin{array}{l}3 \\
0 \\
\frac{3}{4} \\
3 \\
0 \\
\frac{0}{d} \\
\frac{\pi}{1} \\
\frac{1}{t}\end{array}$ & 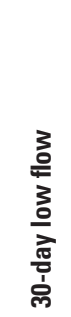 & 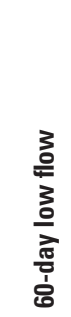 & 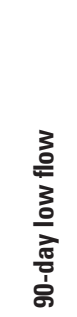 & 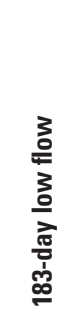 & 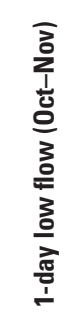 & 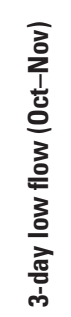 & 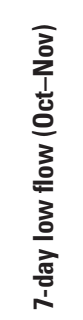 & 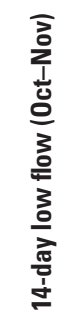 & 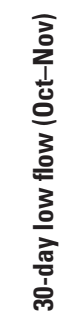 & 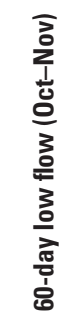 & 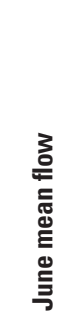 & 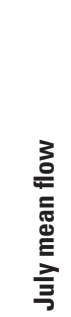 & 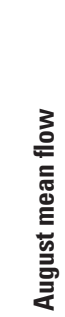 \\
\hline 06799385 & $\begin{array}{l}\text { Pebble Creek at } \\
\text { Scribner, Nebr. }\end{array}$ & 1979-2003 & I & +0.5 & +0.5 & +0.5 & +0.5 & +0.6 & +0.8 & +0.7 & I & I & +0.8 & +0.8 & I & I & I & I & I & I & -18 \\
\hline 06799500 & $\begin{array}{l}\text { Logan Creek } \\
\text { near Uehling, } \\
\text { Nebr. }\end{array}$ & $1941-2003$ & POS & +1.4 & +1.3 & +1.3 & +1.4 & +1.4 & +1.5 & +1.6 & +1.8 & +1.8 & +1.7 & +1.7 & +1.6 & +1.6 & +1.5 & +1.7 & POS & POS & POS \\
\hline 06800000 & $\begin{array}{l}\text { Maple Creek near } \\
\text { Nickerson, } \\
\text { Nebr. }\end{array}$ & $1952-2003$ & +1.2 & +.2 & +.2 & +.2 & +.2 & +.3 & +.4 & +.6 & +.6 & +.7 & +.4 & +.4 & +.4 & +.4 & +.5 & +.6 & I & +3.4 & +1.6 \\
\hline 06800500 & $\begin{array}{l}\text { Elkhorn River } \\
\text { at Waterloo, } \\
\text { Nebr. }\end{array}$ & 1928-2003 & +15 & +4.1 & +4.3 & +4.4 & +4.8 & +5.4 & +6 & +6.6 & +7.1 & +7.4 & +5.6 & +5.5 & +6.1 & +6.3 & +6.2 & +7.8 & +22 & +22 & +9.3 \\
\hline 06803555 & $\begin{array}{l}\text { Salt Creek at } \\
\text { Greenwood, } \\
\text { Nebr. }\end{array}$ & $1952-2003$ & +2.9 & +1.7 & +1.7 & +1.7 & +1.7 & +1.7 & +2 & +2 & +2 & +2.2 & +1.5 & +1.5 & +1.5 & +1.7 & +2 & +2.6 & I & I & I \\
\hline 06804700 & $\begin{array}{l}\text { Wahoo Creek at } \\
\text { Ashland, Nebr. }\end{array}$ & 1990-2004 & I & I & I & I & I & I & I & I & I & I & I & I & I & I & I & I & I & -28 & I \\
\hline 06805500 & $\begin{array}{l}\text { Platte River at } \\
\text { Louisville, } \\
\text { Nebr. }\end{array}$ & 1954-2004 & +70 & +24 & +27 & +27 & +28 & +35 & +45 & +54 & +57 & +59 & +53 & +55 & +58 & +57 & +63 & +69 & I & +96 & +57 \\
\hline
\end{tabular}


Elkhorn River at Norfolk; North Fork Elkhorn River near Pierce; Elkhorn River at West Point; Platte River near Leshara; and Wahoo Creek at Ashland.

Sites with positive trends in June mean flows included: Elkhorn River near Atkinson; Elkhorn River at Neligh; Logan Creek near Uehling; and Elkhorn River at Waterloo. Negative trends were indicated at the Platte River near Leshara site. Trends were not significant at: Platte River near Duncan; Platte River at North Bend; Elkhorn River at Ewing; South Fork Elkhorn River at Ewing; Elkhorn River at Norfolk; North Fork Elkhorn River near Pierce; Elkhorn River at West Point; Pebble Creek at Scribner; Maple Creek near Nickerson; Salt Creek at Greenwood; Wahoo Creek at Ashland; and Platte River at Louisville.

Sites with positive trends in July mean flows included: South Fork Elkhorn River at Ewing; Elkhorn River at Neligh; Elkhorn River at West Point; Logan Creek near Uehling; Maple Creek near Nickerson; Elkhorn River at Waterloo; and Platte River at Louisville. Negative trends were indicated at: Platte River near Leshara; Elkhorn River near Atkinson; and Wahoo Creek at Ashland. Trends were not significant at: Platte River near Duncan; Platte River at North Bend; Elkhorn River at Ewing; Elkhorn River at Norfolk; North Fork Elkhorn River near Pierce; Pebble Creek at Scribner; and Salt Creek at Greenwood.

Sites with positive trends in August mean flows included: Platte River near Duncan; Logan Creek near Uehling; Maple Creek near Nickerson; Elkhorn River at Waterloo; and Platte River at Louisville. Negative trends were indicated at: Platte River near Leshara; Elkhorn River near Atkinson; and Pebble Creek at Scribner. Trends were not significant at: Platte River at North Bend; Elkhorn River at Ewing; South Fork Elkhorn River at Ewing; Elkhorn River at Neligh; Elkhorn River at Norfolk; North Fork Elkhorn River near Pierce; Elkhorn River at West Point; Salt Creek at Greenwood; and Wahoo Creek at Ashland.

In the Elkhorn River basin, sites upstream from Norfolk, Nebraska, had fewer significant trends, whereas, generally, sites downstream from Norfolk and sites in the Platte River and Salt Creek basins showed positive trends. Positive trends for streamflow during extended low-flow periods indicated that there generally is increasing water availability during the lowest-flow periods of the year. Significant negative trends for flows during low-flow periods resulted only for the Leshara streamflow-gaging station, where the period analyzed was limited by a short period of record.

\section{Streamflows Not Exceeding Instream- Flow Criteria Levels}

Historical Platte River streamflow records for the streamflow-gaging station at Louisville, Nebraska, were used to determine the number of days per water year that would not have satisfied criteria flows corresponding to the minimum requirements of instream Appropriation A-17331 before it was filed in 1993. The following minimum criteria for the instream flow appropriation were compared to the historical record: $3,700 \mathrm{ft}^{3} / \mathrm{s}$ for October through December; $3,100 \mathrm{ft}^{3} / \mathrm{s}$ for January; $3,700 \mathrm{ft}^{3} / \mathrm{s}$ for February through July; $3,500 \mathrm{ft}^{3} / \mathrm{s}$ for August; and 3,200 ft $\mathrm{ft}^{3} / \mathrm{s}$ for September. Intervals of consecutive days for which the daily mean streamflow was less than or equal to the level corresponding to the respective criteria were determined for the Platte River at Louisville streamflowgaging station for WY 1953 through 2004.

The number of days per water year for which the daily mean streamflow was less than or equal to minimum criteria corresponding to instream flow Appropriation A-17331 are listed in table 8. For the period of record before 1993, the median number of days that did not exceed the minimum flow level was 120 days per water year, with 39 days during the July 1-August 31 season, and 76 days during the May 1-September 30 season (table 9).

The intervals during which daily mean streamflows of the Platte River at Louisville streamflow-gaging station failed to exceed the minimum flow criteria levels of Appropriation A-17331 were further summarized (table 9). During WY 1993 through 2004, 48 separate intervals of streamflow less than the criteria levels occurred. Of these intervals, 67 percent were 3 days or greater in duration, and 40 percent were 7 days or greater in duration. The median duration of intervals when streamflow was less than the criteria values was 4 consecutive days.

During 1993 through 2004, daily mean flows of the Platte River at Louisville streamflow-gaging station failed to satisfy the minimum flow levels required by Appropriation A-17331 on a total of 638 days. Most of these low-flow intervals occurred in summer through early fall. During WY 1993 through 2004, the median annual number of days not exceeding the criteria levels was 21.5 days, with 14 days during the July 1 through August 31 season, and no additional days, on average, during the May 1 through September 30 season.

During WY 1953 through 1992, 458 separate intervals of streamflow less than the criteria levels occurred. Of these, 61 percent were 3 days or greater in duration, and 38 percent were 7 days or greater in duration. The median duration of intervals of flow less than the criteria levels was 4 consecutive days. The longest interval of consecutive daily mean streamflow below Appropriation A-17331 criteria levels occurred during WY 1976 and 1977, when 143 consecutive days were recorded as having flows that failed to satisfy the minimum streamflow levels (table 8) required by Appropriation A-17331 (not enacted until 1998). 
Table 8. Summary by water year of intervals in which daily mean streamflow at the U.S. Geological Survey streamflow-gaging station on the Platte River at Louisville, Nebraska (06805500), did not satisfy instream-flow criteria levels, 1953-2004.

[ $\mathrm{ft}^{3} / \mathrm{s}$, cubic feet per second; instream-flow criteria levels are as follows: $3,700 \mathrm{ft}^{3} / \mathrm{s}$ during October through December; $3,500 \mathrm{ft}^{3} / \mathrm{s}$ in January; $3,700 \mathrm{ft}^{3} / \mathrm{s}$ from February through July; 3,500 $\mathrm{ft}^{3} / \mathrm{s}$ in August; 3,200 ft $3 / \mathrm{s}$ in September]

\begin{tabular}{|c|c|c|c|c|}
\hline Water year & $\begin{array}{l}\text { Total number of days } \\
\text { in non-exceedance } \\
\text { intervals }\end{array}$ & $\begin{array}{l}\text { Non-exceedance days } \\
\text { from July } 1 \text { through } \\
\text { August } 31 \text { (percent } \\
\text { of 2-month period in } \\
\text { parentheses) }\end{array}$ & $\begin{array}{l}\text { Non-exceedance days } \\
\text { from May } 1 \text { through } \\
\text { September } 30 \text { (percent } \\
\text { of } 5 \text {-month period in } \\
\text { parentheses) }\end{array}$ & $\begin{array}{l}\text { Longest consecutive non- } \\
\text { exceedance period beginning in } \\
\text { water year (number of days) }\end{array}$ \\
\hline 1953 & 95 & $58(95)$ & $95(63)$ & 08/10/53-11/10/53 (93) \\
\hline 1954 & 163 & $53(87)$ & $86(57)$ & $08 / 26 / 54-10 / 28 / 54$ (64) \\
\hline 1955 & 247 & $59(97)$ & $129(85)$ & 07/17/55-11/23/55 (130) \\
\hline 1956 & 253 & $56(92)$ & $124(82)$ & 09/06/56-12/29/56 (115) \\
\hline 1957 & 197 & $39(64)$ & $60(39)$ & $01 / 09 / 57-02 / 12 / 57$ (35) \\
\hline 1958 & 96 & $17(28)$ & $45(30)$ & $09 / 20 / 58-11 / 14 / 58(56)$ \\
\hline 1959 & 185 & $33(54)$ & $71(47)$ & 08/19/59-09/19/59 (32) \\
\hline 1960 & 116 & $29(48)$ & $47(31)$ & $10 / 13 / 59-10 / 30 / 59(18)$ \\
\hline 1961 & 148 & $59(97)$ & $79(52)$ & 07/02/61-08/21/61 (51) \\
\hline 1962 & 81 & $13(21)$ & $27(18)$ & $12 / 10 / 61-12 / 25 / 61(16)$ \\
\hline 1963 & 121 & $60(98)$ & $91(60)$ & $07 / 02 / 63-08 / 30 / 63(60)$ \\
\hline 1964 & 111 & $37(61)$ & $52(34)$ & $10 / 04 / 63-11 / 05 / 63(33)$ \\
\hline 1965 & 145 & $33(54)$ & $39(26)$ & $10 / 01 / 64-11 / 16 / 64(47)$ \\
\hline 1966 & 97 & $41(67)$ & $77(51)$ & $01 / 20 / 66-02 / 04 / 66(16)$ \\
\hline 1967 & 180 & $22(36)$ & $82(54)$ & $04 / 18 / 67-05 / 31 / 67$ (44) \\
\hline 1968 & 142 & $56(92)$ & $97(64)$ & 07/07/68-09/01/68 (57) \\
\hline 1969 & 113 & $36(59)$ & $64(42)$ & 08/03/69-09/01/69 (30) \\
\hline 1970 & 108 & $54(89)$ & $80(53)$ & 07/09/70-09/18/70 (72) \\
\hline 1971 & 122 & $45(74)$ & $75(49)$ & 07/18/71-10/19/71 (94) \\
\hline 1972 & 119 & $39(64)$ & $73(48)$ & 08/12/72-09/10/72 (30) \\
\hline 1973 & 65 & $30(49)$ & $34(22)$ & 08/20/73-09/04/73 (16) \\
\hline 1974 & 100 & $62(100)$ & $99(65)$ & 06/24/74-10/30/74 (129) \\
\hline 1975 & 170 & $52(85)$ & $95(63)$ & 07/28/75-11/06/75 (102) \\
\hline 1976 & 198 & $62(100)$ & $121(80)$ & 06/30/76-11/19/76 (143) \\
\hline 1977 & 153 & $38(62)$ & $40(26)$ & $11 / 27 / 76-01 / 13 / 77$ (48) \\
\hline 1978 & 126 & $50(82)$ & $83(55)$ & $09 / 22 / 78-10 / 25 / 78$ (34) \\
\hline 1979 & 206 & $36(59)$ & $77(51)$ & $11 / 29 / 78-02 / 26 / 79(90)$ \\
\hline 1980 & 138 & $59(97)$ & $89(59)$ & $08 / 20 / 80-10 / 16 / 80(58)$ \\
\hline 1981 & 206 & $35(57)$ & $101(66)$ & 07/04/81-07/29/81 (26) \\
\hline 1982 & 48 & $15(25)$ & $15(10)$ & $01 / 08 / 82-01 / 20 / 82(13)$ \\
\hline 1983 & 5 & $0(0)$ & $0(0)$ & $12 / 29 / 82-01 / 02 / 83(5)$ \\
\hline 1984 & 24 & $13(21)$ & $18(12)$ & 08/12/84-08/23/84 (12) \\
\hline 1985 & 18 & $13(21)$ & $18(12)$ & 07/10/85-07/18/85 (9) \\
\hline 1986 & 7 & $0(0)$ & $0(0)$ & $11 / 21 / 85-11 / 26 / 85(6)$ \\
\hline 1987 & 14 & $14(23)$ & $14(9)$ & 07/26/87-08/07/87 (13) \\
\hline 1988 & 97 & $52(85)$ & $84(55)$ & 08/20/88-09/15/88 (27) \\
\hline 1989 & 121 & $47(77)$ & $104(68)$ & 07/23/89-09/03/89 (43) \\
\hline 1990 & 111 & $44(72)$ & $74(49)$ & $08 / 23 / 90-10 / 23 / 90(62)$ \\
\hline 1991 & 136 & $52(85)$ & $82(54)$ & 07/18/91-10/31/91 (106) \\
\hline
\end{tabular}


Table 8. Summary by water year of intervals in which daily mean streamflow at the U.S. Geological Survey streamflow-gaging station on the Platte River at Louisville, Nebraska (06805500), did not satisfy instream-flow criteria levels, 1953-2004.—Continued

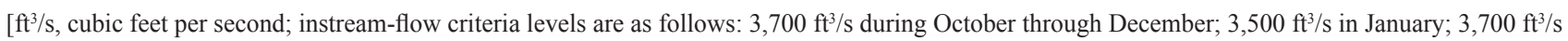
from February through July; 3,500 ft $\mathrm{ft}^{3} / \mathrm{s}$ in August; 3,200 ft³ $/ \mathrm{s}$ in September]

\begin{tabular}{ccccc}
\hline Water year & $\begin{array}{c}\text { Total number of days } \\
\text { in non-exceedance } \\
\text { intervals }\end{array}$ & $\begin{array}{c}\text { Non-exceedance days } \\
\text { from July 1 through } \\
\text { August 31 (percent } \\
\text { of 2-month period in } \\
\text { parentheses) }\end{array}$ & $\begin{array}{c}\text { Non-exceedance days } \\
\text { from May 1 through } \\
\text { September 30 (percent } \\
\text { of 5-month period in } \\
\text { parentheses) }\end{array}$ & $\begin{array}{c}\text { Longest consecutive non- } \\
\text { excedance period beginning in } \\
\text { water year (number of days) }\end{array}$ \\
\hline 1993 & 0 & $0(0)$ & $0(0)$ & None \\
1994 & 4 & $3(5)$ & $4(3)$ & $08 / 30 / 94-08 / 31 / 94(2)$ \\
1995 & 15 & $9(15)$ & $9(6)$ & $08 / 14 / 95-08 / 22 / 95(9)$ \\
1996 & 18 & $0(0)$ & $0(0)$ & $01 / 21 / 96-01 / 27 / 96(7)$ \\
1997 & 25 & $19(31)$ & $19(13)$ & $07 / 24 / 97-08 / 11 / 97(19)$ \\
1998 & 0 & $0(0)$ & $0(0)$ & None \\
1999 & 1 & $0(0)$ & $70(46)$ & $12 / 23 / 98-12 / 23 / 98(1)$ \\
2000 & 56 & $27(44)$ & $35(23)$ & $08 / 06 / 00-09 / 28 / 00(54)$ \\
2001 & 52 & $28(46)$ & $121(80)$ & $07 / 31 / 01-08 / 17 / 01(18)$ \\
2002 & 124 & $57(93)$ & $107(70)$ & $06 / 21 / 02-08 / 21 / 02(62)$ \\
2003 & 146 & $51(84)$ & $75(49)$ & $07 / 13 / 03-09 / 11 / 03(61)$ \\
2004 & 197 & $40(66)$ & $09 / 17 / 03-11 / 03 / 03(48)$ \\
\hline
\end{tabular}

\section{Quality Assurance of Streamflow Measurements and Stage Records for the Platte River at Louisville, Nebraska}

For this study, the quality-assurance measures used for processing and publishing of stage and streamflow data at the Platte River at Louisville, Nebraska, streamflow-gaging station, were reviewed and summarized. Discharge, or streamflow, measurement data were retrieved from the USGS National Water Information System database (http://waterdata.usgs.gov/nwis) and summarized.

The USGS streamflow-gaging station on the Platte River at Louisville, Nebraska, is located on the left bank approximately $50 \mathrm{ft}$ upstream from the State Highway 50 bridge (fig. 1). The streamflow-gaging station presently collects stage data at 15-minute intervals and is included in the USGS Nebraska Water Science Center Surface-Water Quality-Assurance Plan (P.J. Soenksen, U.S. Geological Survey, written commun., 2006). A reference wire-weight gage is located on the downstream side of the Highway 50 bridge. The station instrumentation is capable of recording stage data accurately to \pm 0.01 $\mathrm{ft}$. Occasional instrumentation drift requires recalibration. Corrections, called datum corrections, are applied to stage data values recorded by the datalogger to compensate for differences between the readings of the reference and recording gages (Rantz and others, 1982a). Periodically, level surveys are conducted to check the reference gage for vertical movement (Rantz and others, 1982a). Datum corrections for the periods between level survey checks are applied to the stage record to account for these changes, if vertical movement exceeds $0.02 \mathrm{ft}$ (Rantz and others, 1982a).

Standard USGS protocols require hydrographers to assign qualitative assessments of conditions affecting accuracy to individual discharge measurements - excellent, good, fair, or poor (Rantz and others, 1982a). These qualitative ratings are determined by the hydrographer based on subjective evaluation of an objectively determined suite of factors affecting measurement accuracy, which includes: number and distribution of verticals, average velocity, uniformity of flow, regularity and firmness of channel bottom, steadiness of stage and discharge during the measurement, and presence or absence of ice or debris in the flow (Sauer and Meyer, 1992).

Since the Platte River at Louisville streamflow-gaging station was established in May of 1953, more than 550 measurements have been made using standard USGS discharge measurement techniques and equipment (Corbett and others, 1943; Rantz and others, 1982a). All measurement computations were checked by USGS personnel for mathematical accuracy following methods listed in Kennedy (1983).

Discharge measurements collected before the start of WY 1985 were not computerized; therefore, the summaries of measurements were limited to WY 1985 through 2004. Of the 268 direct discharge measurements collected during that period, 93 percent of measurements were collected from the Highway 50 bridge; the remaining measurements were collected by wading or as ice measurements. The measurements of streamflows within 10 percent of the range of flows not exceeding the present criteria for minimum instream flows were summarized 
Table 9. Comparison by period of intervals in which daily mean streamflow at the U.S. Geological Survey streamflow-gaging station on the Platte River at Louisville, Nebraska (station 06805500), did not exceed instreamflow criteria levels, 1953-2004.

[ft 3 s, cubic feet per second; instream-flow criteria levels are as follows: $3,700 \mathrm{ft}^{3} / \mathrm{s}$ during October through December; $3,500 \mathrm{ft}^{3} / \mathrm{s}$ in January; 3,700 ft $3 / \mathrm{s}$ from February through July; 3,500 ft $3 / \mathrm{s}$ in August; 3,200 ft $3 / \mathrm{s}$ in September]

\begin{tabular}{|c|c|c|c|}
\hline \multirow[b]{2}{*}{ Summary statistic } & \multicolumn{3}{|c|}{ Summary time period, in water years } \\
\hline & 1953-2004 & 1953-1992 & 1993-2004 \\
\hline \multicolumn{4}{|c|}{ Intervals } \\
\hline Total number of intervals & 506 & 458 & 48 \\
\hline Average length of each interval, in days & 10 & 11 & 10 \\
\hline Median length of each interval, in days & 4 & 4 & 4 \\
\hline Intervals 3 or more days in length, as percentage of period & 61 & 61 & 67 \\
\hline Intervals 7 or more days in length, as percentage of period & 38 & 38 & 40 \\
\hline \multicolumn{4}{|c|}{ Non-exceedance days } \\
\hline Total number of non-exceedance days & 5,469 & 4,831 & 638 \\
\hline Median number of non-exceedance days per water year & 112 & 120 & 21.5 \\
\hline $\begin{array}{l}\text { Median number of non-exceedance days from July } 1 \text { through } \\
\text { Aug. } 31 \text { per water year }\end{array}$ & 36.5 & 39 & 14 \\
\hline $\begin{array}{l}\text { Median number of non-exceedance days from May } 1 \text { through } \\
\text { Sept. } 30 \text { per water year }\end{array}$ & 72 & 76 & 14 \\
\hline
\end{tabular}

(table 10). During WY 1985 through 2004, 55 measurements of discharges less than $4,000 \mathrm{ft}^{3} / \mathrm{s}$ were made. The lowest flow measured during WY 1985 through 2004 was $911 \mathrm{ft}^{3} / \mathrm{s}$ on August 7, 2002. The highest flow measured during WY 1985 through 2004 was 125,000 ft³ $/ \mathrm{s}$ on March 10, 1993. Many measurements at this site, especially low-flow measurements, required the use of horizontal-angle coefficients to adjust measured velocities when the flowlines were not normal to the measuring section. Qualitative ratings indicate that 84 percent of measurements made at discharges less than $4,000 \mathrm{ft}^{3} / \mathrm{s}$ and 87 percent overall are considered to be accurate to within 5 percent of the true discharge.
For the Louisville streamflow-gaging station, stagedischarge relations were developed, modified, and applied following standard procedures described in Rantz and others, (1982a; 1982b); Kennedy, (1983); and Kennedy, (1984). These stage-discharge relations can be represented by tables, and also graphically as rating curves. Here the Platte River is a wide channel with a sand and gravel bed that shifts readily with changes in stage; therefore, stage-discharge rating tables are adjusted routinely by applying adjustments to stage values known as ratings shifts and as described by Kennedy (1984). During WY 1985 through 2004, five rating curves have been developed for the Platte River at Louisville streamflow-gaging

Table 10. Summary of discharge measurements from water years 1985 through 2004 for U.S. Geological Survey streamflow-gaging station on the Platte River at Louisville, Nebraska (station 06805500).

[ft $\mathrm{ft}^{3} / \mathrm{s}$, cubic feet per second; \%, percent; columns may not sum to $100 \%$ because of rounding]

\begin{tabular}{lcc}
\hline $\begin{array}{c}\text { Qualitative rating of conditions } \\
\text { affecting measurement accuracy }\end{array}$ & $\begin{array}{c}\text { Measured discharge less than } \\
\mathbf{4 , 0 0 0} \mathbf{~ f t}^{3} / \mathbf{s} \text { (percent of all such mea- } \\
\text { surements in parentheses) }\end{array}$ & $\begin{array}{c}\text { All discharge measurements } \\
\text { within period (percent of all mea- } \\
\text { surements in parentheses) }\end{array}$ \\
\hline $\begin{array}{l}\text { Excellent (within 2 percent of the } \\
\text { actual "true" discharge) }\end{array}$ & $1(2 \%)$ & $2(1 \%)$ \\
Good (within 5 percent) & $45(82 \%)$ & $231(86 \%)$ \\
$\begin{array}{l}\text { Fair (within 8 percent) } \\
\text { Poor (measured discharge 8 percent } \\
\text { greater or less than the actual } \\
\text { discharge) }\end{array}$ & $4(7 \%)$ & $23(9 \%)$ \\
Not rated & $3(5 \%)$ & $10(4 \%)$ \\
\hline
\end{tabular}


station. Shift adjustments among all measurements during the period ranged from -1.95 to $1.50 \mathrm{ft}$. Shift adjustments of large magnitudes generally were associated with ice measurements or moderate to higher streamflows capable of scouring and filling the sandy streambed within a short time period during and after the higher flow occurred. For measurements of discharges less than $4,000 \mathrm{ft}^{3} / \mathrm{s}$ not affected by ice, shift adjustments ranged from 0.18 to $0.40 \mathrm{ft}$. The shift adjustments for measurements of flows less than $4,000 \mathrm{ft}^{3} / \mathrm{s}$ were applied routinely to adjust the rating table for the effects of shifting sand and seasonal changes in vegetation.

The USGS follows standard procedures for collecting and adjusting continuous stage data and periodic discharge data, and for processing those data to produce continuous records of discharge. Those procedures include, but are not limited to, making stage-reference measurements to adjust stage records, documenting non-perpendicular flow angles in the cross section to adjust discharge measurements accordingly, and comparing computed discharge records with those for other stations that are hydrologically similar. The discharge records are assigned a qualitative rating of accuracy based on a number of factors, including some of those listed in Sauer and Meyer (1992), and then are independently checked and reviewed. On this basis, the published discharge records for the Platte River at Louisville streamflow-gaging station are considered to be accurate within the assigned limits indicated by the qualitative rating (table 10) (P.J. Soenksen, U.S. Geological Survey, written commun., 2008).

\section{Summary and Conclusions}

Instream-flow protection is a complex legislative and technical issue. The Nebraska Department of Natural Resources established appropriations on the Platte River to provide instream-flow protection primarily to maintain the fish community, whooping crane roost habitat, and wet meadows. In 2005, the USGS, in cooperation with the Upper Elkhorn Natural Resources District and the Lower Elkhorn Natural Resources District, initiated a cooperative study to review the methods used to calculate the streamflow requirements for the Nebraska Game and Parks Commission instream appropriation on the Platte River in Nebraska, to review the historical streamflow records for the Platte River at Louisville streamflow-gaging station, and to indicate the quality of the data for the Platte River at Louisville streamflow-gaging station for managing flows on the appropriated reach of the Platte River from its confluence with the Elkhorn River to the mouth.

Confluences with two principal tributaries of the Platte River - the Elkhorn River and Salt Creek - are located within the reach regulated by Appropriation A-17331. The Elkhorn River flows east-southeast from the Sand Hills in north-central Nebraska through the glaciated rolling hills of northeast Nebraska to its confluence with the Platte River just downstream from Fremont, Nebraska. Streamflows in the Sand Hills in the western part of the drainage basin originate principally from ground water; streamflows in the eastern part of the basin generally are more variable and responsive to precipitation events. Salt Creek flows into the Platte River from the south just downstream from the confluence of the Elkhorn and Platte Rivers.

Surface-water use in the Elkhorn River basin includes irrigation, livestock, and recreational supplies. As of January 30, 2005, 362 surface-water appropriations were listed in the NDNR database with application dates later than 1970 (Nebraska Department of Natural Resources, 2005). These appropriations are listed as irrigating about 29,000 acres in the Elkhorn River basin.

The Platte River has a drainage area of about $71,000 \mathrm{mi}^{2}$ upstream from the USGS streamflow-gaging station at Louisville, Nebraska. Extremes for discharge at this station have ranged from a low of $131 \mathrm{ft}^{3} / \mathrm{s}$ to a high of $124,000 \mathrm{ft}^{3} / \mathrm{s}$, and the mean discharge (1953 through 2005) was $6,966 \mathrm{ft}^{3} / \mathrm{s}$.

PHABSIM, a habitat simulation model, and other estimation methodologies had been used in previous studies to estimate instream-flow requirements of the fish community of the lower Platte River. To estimate instream-flow requirements, PHABSIM uses hydraulic principles to simulate water depths and velocities on the basis of empirical data, such as channel geometry, substrate classification, vegetative cover indexes, and other measurements collected along the stream reach. In PHABSIM, criteria for favorable habitat are expressed as suitability index curves, which relate physical features to a microhabitat-suitability score.

Efforts to estimate instream-flow requirements using PHABSIM and other estimation methodologies resulted in the flow magnitude requests in five applications for instream appropriations filed by NGPC in 1993 for the purpose of maintaining fish and wildlife in the central and eastern Platte River. Analysis of PHABSIM results for all fish species indicated that habitat availability rapidly declines as flows fall below $2,000 \mathrm{ft}^{3} / \mathrm{s}$.

Based on results of PHABSIM analysis, NGPC requested an instream-flow appropriation (Appropriation A-17331) of 3,700 $\mathrm{ft}^{3} / \mathrm{s}$ for the reach from the Elkhorn River to the mouth of the Platte River. This appropriation was granted with modifications in 1998, having been modified to account for the Metropolitan Utilities District's senior water right of $500 \mathrm{ft}^{3} / \mathrm{s}$ and to meet a 20-percent exceedance flowduration threshold.

Statistical summaries were computed for 17 selected streamflow-gaging stations in the study area for an analysis of streamflow characteristics. Streamflow records from 1928 through 2004 in the Platte River and Elkhorn River basins and from the 1950s through 2004 in the Salt Creek basin were summarized. From WY 1954 through 1992, an average of 19 percent of the mean annual flows at the Platte River at Louisville streamflow-gaging station was measured at the Elkhorn River at Waterloo streamflow-gaging station. From WY 1993 through 2004, an average of 25 percent of the mean annual flow at the Platte River at Louisville streamflow-gaging station 
was measured at the Elkhorn River at Waterloo streamflowgaging station.

Generally, the streamflow of the Platte River at Louisville streamflow-gaging station is the sum of streamflows of the Elkhorn River, Salt Creek, Wahoo Creek, and the Platte River at Ashland, Nebraska; plus ungaged inflows minus outflows between the streamflow-gaging stations on these streams and the Louisville streamflow-gaging station. Annual mean flow of the Elkhorn River at Waterloo streamflow-gaging station ranged from 12 to 30 percent of the annual mean flow at the Platte River at Louisville streamflow-gaging station during WY 1954 through 2004, with a standard deviation of 4.3 percent.

Daily mean streamflows were used to identify extreme low-flow periods of various durations, or $n$-day low flows. Annual low-flow values were computed as the minimum mean flows for $n$ consecutive days during a climatic year (April to March), for durations of 1, 3, 7, 14, 30, 60, and 183 days for 17 selected streamflow-gaging stations in the study area.

Kendall's Tau test was used to detect temporal trends in selected flow statistics. The streamflow values were not adjusted to remove the precipitation variation. In this study, analysis of the results of Kendall's Tau for trends in annual streamflow indicated significant $p$-values and the presence of positive trends for the period of record at eight sites - Platte River near Duncan; Platte River at North Bend; Elkhorn River at Neligh; Logan Creek near Uehling; Maple Creek near Nickerson; Elkhorn River at Waterloo; Salt Creek at Greenwood; and Platte River at Louisville. Generally, fewer significant positive trends resulted for sites in the Elkhorn River basin upstream from Norfolk, whereas results for sites in the Elkhorn River basin downstream from Norfolk, on the Platte River, and in Salt Creek basin, indicated generally positive trends for annual mean flow and $n$-day low flows.

Historical streamflow records at the USGS streamflowgaging station at Platte River at Louisville, Nebraska, were used to determine the number of days per water year that discharge would not have satisfied instream-flow criteria levels corresponding to the minimum requirements of Appropriation A-17331 before it was filed. The following criteria levels for instream flow were compared to the historical record: 3,700 $\mathrm{ft}^{3} / \mathrm{s}$ for October through December; 3,500 $\mathrm{ft}^{3} / \mathrm{s}$ for January; $3,700 \mathrm{ft}^{3} / \mathrm{s}$ for February through July; $3,500 \mathrm{ft}^{3} / \mathrm{s}$ for August; and $3,200 \mathrm{ft}^{3} / \mathrm{s}$ for September. For the period of record before 1993, the median number of days that discharge failed to exceed the criteria levels was 120 days per water year, with 39 days during the July 1 -August 31 period, and 76 days during the May 1-September 30 period.

Of the 458 periods of consecutive days in which the daily mean flow did not exceed criteria levels corresponding to instream flow appropriation A-17331 at the Platte River at Louisville streamflow-gaging station (WY 1953-1992), 61 percent were 3 days or greater in duration, and 38 percent were 7 days or greater in duration. The median duration of flow less than the threshold was 4 consecutive days for WY 1953-92 and also for 1993 through 2004. The longest period of flow below the threshold levels occurred during WY 1976 through 1977, when for 143 consecutive days the recorded daily mean streamflow was at or below the minimum streamflow later required by Appropriation A-17331.

Since 1993, daily mean flows for 638 days have failed to exceed the minimum flow requirements of A-17331 at the Platte River at Louisville, Nebraska, USGS streamflow-gaging station. Most of these low-flow periods occurred in summer through early fall. For WY 1993 through 2004, the median number of days that did not exceed the flow criteria was about 21 days per water year, with 14 days during the July 1-August 31 period, and no additional days during the May 1-September 30 period.

Since the Platte River at Louisville streamflow-gaging station was established in May 1953, more than 550 streamflow measurements have been made. Summaries of streamflow measurements were limited to WY 1985 through 2004. Of the 268 discharge measurements during that period, 93 percent were collected from the Highway 50 bridge, whereas the remaining measurements were made in or on the water (wading or ice measurements). During WY 1985 through 2004, 55 measurements of discharges less than $4,000 \mathrm{ft}^{3} / \mathrm{s}$ were made, with the lowest flow measured as $911 \mathrm{ft}^{3} / \mathrm{s}$ on August 7, 2002. Qualitative ratings indicate that 84 percent of measurements made at discharges less than $4,000 \mathrm{ft}^{3} / \mathrm{s}$, and 87 percent overall, are considered to be accurate to within 5 percent of the true discharge.

Stage-discharge relations were developed as rating tables and rating curves, modified, and applied following standard USGS procedures, and stage-discharge rating tables were routinely adjusted by applying adjustments to stage called ratings shifts. During WY 1985 through 2004, shift adjustments ranged from -1.95 to $1.50 \mathrm{ft}$ among all measurements, and from -0.18 to $0.40 \mathrm{ft}$ for measurements of discharge less than $4,000 \mathrm{ft}^{3} / \mathrm{s}$.

\section{References Cited}

Aiken, J.D., 1989, Instream appropriations in Nebraska, in MacDonnell, L. J., Rice, T.A., and Shupe, S. J, eds., Instream flow protection: Boulder, Colo., Natural Resources Law Center, Univ. of Colorado School of Law, p. 313-330.

Annear, T.C., Chisholm, I.M., Beecher, H.A., Locke, Allan, and 12 others, 2004, Instream flows for riverine resource stewardship (rev. ed.): Cheyenne, Wyo., Instream Flow Council, 268 p.

Bentall, Ray, and others, 1971, Water supplies and the landThe Elkhorn River basin of Nebraska: Lincoln, University of Nebraska, Conservation and Survey Division, Resource Atlas 1, $51 \mathrm{p}$. 
Bovee, K.D., 1982, A guide to stream habitat analysis using the Instream Flow Incremental Methodology: U.S. Fish and Wildlife Service, Instream Flow Information Paper 12, FWS/OBS-82-26, $248 \mathrm{p}$.

Bovee, K.D., Lamb, B.L., Bartholow, J.M., Stalnaker, C.B, Taylor, J.G., and Henriksen, J.A., 1998, Stream habitat analysis using the Instream Flow Incremental Methodology: U.S. Geol. Survey Biological Resources Discipline Information and Technology Report USGS/BRD-1998-0004, $131 \mathrm{p}$.

Corbett, D.M., and others, 1943, Stream-gaging procedure: U.S. Geological Survey Water-Supply Paper 888, p. 224-228.

Espegren, G. D., 1996, Development of instream flow recommendations in Colorado using R2CROSS, Denver, CO, Colorado Water Conservation Board, $34 \mathrm{p}$.

Hardy and Associates, 1992, Instream flow analyses of the central Platte River: Lincoln, Nebr., Nebraska Game and Parks Commission, 54 p., appendices.

Helsel, D.R., and Hirsh, R.M., 1992, Statistical methods in water resources: New York, Elsevier, 529 p.

Henriksen, J.A., Heasley, John, Kennen, J.G., and Nieswand, Steven, 2006, Users' manual for the hydroecological integrity assessment process software (including the New Jersey Assessment Tools): U.S. Geological Survey Open-File Report 2006-1093, 71 p.

Interagency Advisory Committee on Water Data, 1982, Guidelines for determining flood flow frequency: Reston, Virginia, U.S. Geological Survey, Office of Water Data Coordination, Bulletin 17-B of the Hydrology Subcommittee, $183 \mathrm{p}$.

Jorde, Klaus, Schneider, Matthias, Peter, Armin, and Zoellner, Frank, 2001, Fuzzy based models for the evaluation of fish habitat quality and instream flow assessment, in International Symposium on Environmental Hydraulics, 3rd, Tempe, Ariz., 2001, Proceedings: Madrid, Int. Assoc. of Hydr. Eng. and Research (IAHR), p.1.

Karl, T.R., and Knight, R.W., 1998, Secular trends of precipitation amount, frequency, and intensity in the United States: Bulletin of the American Meteorological Society, v. 79, p. 231-242.

Kennedy, E.J., 1983, Computation of continuous records of streamflow: U.S. Geological Survey Techniques of WaterResources Investigations, book 3, chap. A13, 53 p.

Kennedy, E.J., 1984, Discharge ratings at gaging stations: U.S. Geological Survey Techniques of Water-Resources Investigations, book 3, chapter A10, 59 p.
Lins, H.F., and Slack, J.R., 1999, Streamflow trends in the United States: Geophysical Research Letters, v. 26, p. $227-$ 230.

Lumb, A.M., Kittle, J.L., Jr., and Flynn, K.M., 1990, Users manual for ANNIE, a computer program for interactive hydrologic analyses and data management: U.S. Geological Survey Water-Resources Investigations Report 89-4080, $236 \mathrm{p}$.

McCabe, G.J., and Wolock, D.M., 2002, A step increase in streamflow in the conterminous United States: Geophysical Research Letters, v. 29, no. 24, p. 2,185-2,188.

Milhous, R.T., Wegner, D.L., and Waddle, T.J., 1984, User's guide to the Physical Habitat Simulation System (rev. ed.): U.S. Fish and Wildlife Service, Instream Flow Information Paper 11, FWS/OBS-81/43, 475 p.

Milly, P.C.D., 2005, Trends in the water budget of the Mississippi River Basin, 1949-1997: U. S. Geological Survey Fact Sheet 2005-3020, 2 p.

Nebraska Department of Natural Resources, 1998, In the matter of applications A-17329 through A-17333, Water Divisions 1-A, 2-A and 2-B; Order: Lincoln, Nebraska Department of Water Resources, accessed January 30, 2005, at http://www.dnr.state.ne.us/legal/decision1.htm

Nebraska Department of Natural Resources, 2005, Nebraska surface water rights data: Lincoln, Nebr Dept. of Natural Resour., retrieval of data on the web, accessed January 30, 2005, at http://dnrdata.dnr.ne.gov/SWRCombined/ SelectSearchOptions.aspx

Peters, E.J., Holland, R.S., Callam, M.A., and Bunnell, D.L., 1989, Platte River suitability criteria; Habitat utilization, preference, and suitability index criteria for fish and aquatic invertebrates in the lower Platte River: Lincoln, Nebr., Nebraska Game and Parks Commission, 135 p.

Peters, E.J., and Holland, R.S., 1992, Shallow-water fish community abundance and habitat use in the lower Platte River, Nebraska: Lincoln, University of Nebraska, Agriculture Research Division, Journal Series 9109, 24 p.

Poff, N.L., Allan, J.D., Bain, M.B., Karr, J.R., Prestegaard, K.L., Richter, B.D., Sparks, R.E., and Stromberg, J.C., 1997, The natural flow regime-A paradigm for conservation and restoration of river ecosytems: BioScience, v. 47, p. 769-784.

Rantz, S.E., and others, 1982a, Measurement and computation of streamflow-Volume 1, Measurement of stage and discharge: U.S. Geological Survey Water-Supply Paper 2175, $284 \mathrm{p}$.

Rantz, S.E., and others, 1982b, Measurement and computation of streamflow-Volume 2, Computation of discharge: U.S. Geological Survey Water-Supply Paper 2175, 346 p. 
Richter, R.D., Baumgartner, J.V., Wigington, Robert, and Braun, D.P., 1997, How much water does a river need?: Freshwater Biology, v. 37, p. 231-249.

Sauer, V.B., and Meyer, R.W., 1992, Determination of error in individual discharge measurements: U.S. Geological Survey Open-File Report 92-144, 21 p.

Schainost, Steve, Hutchinson, J.L, and Zuerlein, E.J., 1993, Platte River instream flow, fish community Elkhorn River to Missouri River, in Platte River instream flow reports: Lincoln, Nebraska Game and Parks Commission Technical Reports [variously paged].

Tennant, D.L., 1976, Instream flow regimens for fish, wildlife, recreation, and related environmental resources: Fisheries (Bethesda), v. 1, no. 4, p. 6-10.
Twelve Nine, Inc., 1990, Instream-flow (PHABSIM) analysis of the lower Platte River fish species for the Interior Least Tern forage: Lincoln, Nebraska Game and Parks Commission, 25 p., appendices.

U.S. Geological Survey, 2006, Water resources data, Nebraska, water year 2005: U.S. Geological Survey WaterData Report NE-05-1, accessed at http://wdr.water.usgs.gov/ wy2005/search.jsp

Zuerlein, E.J, Hutchinson, J.L., Schainost, Steve, and Lock, Ross, 2001, Instream flow rights for the Platte River-A major tributary of the Missouri River, in Platte River Basin Ecosystem Symposium, 11th, Kearney, Nebr., 2001, Proceedings: Lincoln, Univ. of Nebraska, accessed January 30, 2005, at http://www.ianr.unl.edu/ianr/pwp/PROC2001.htm 

Appendix 1. Streamflow Characteristics 


\section{PLATTE RIVER NEAR DUNCAN, NEBR.}

LOCATION.--Lat $41^{\circ} 22^{\prime} 06^{\prime}$, long 97 $29^{\prime} 43^{\prime \prime}$, in SE 1/4 SW 1/4 sec.12, T.16 N., R.2 W., Platte County, Hydrologic Unit 10200103, on left bank near northwest corner of county bridge, 1.5 mi south of Duncan, and 15.3 mi upstream from Loup River, and at mile 114.

DRAINAGE AREA.--59,300 $\mathrm{mi}^{2}$ of which $4,670 \mathrm{mi}^{2}$ probably is noncontributing.

\section{STREAMFLOW RECORDS}

PERIOD OF RECORD.--June 1895 to December 1909 (irrigation seasons only 1885-1900). July 1910 to December 1911 (gage heights and streamflow measurements only), April 1912 to September 1915, June 1928 to current year. Published as "near Columbus" 1895-1915.

REVISED RECORDS.--WSP 956: 1935. WSP 1390: 1897, 1899-1901, 1903-05, 1929-32, 1935(M), 1936. WDR NE-94-1: Drainage area.

GAGE.--Water-stage recorder with satellite telemetry. Datum of gage is 1,476.82 ft above sea level. June 1895 to December 1909, April 1912 to September 1915, and June to October 1928, non-recording gage at site 7 mi downstream at different datums. Oct. 25, 1928, to Feb. 20, 1935, nonrecording gage, and Feb. 20, 1935 to Mar. 21, 1984, recording gage both at present site at $2.00 \mathrm{ft}$ higher datum. Mar. 22, 1984, to Mar. 4, 1987, at site $300 \mathrm{ft}$ downstream at present datum. Data collection platform at station.

REMARKS.--Records good except for estimated daily streamflows, which are poor. Natural flow of stream affected by transmountain diversions, storage reservoirs, power developments, ground-water withdrawals and diversions for irrigation, and return flow from irrigated areas. EXTREMES FOR PERIOD OF RECORD.--1895-1909, 1912-15, 1928-84: Maximum streamflow observed, 44,100 ft³ June 23, 1905 (gage height, 6.50 feet, site and datum then in use); no flow at times in 1931, 1933-42, 1944, 1952-57, 1959, 1963, 1974, 1976, 1978.

EXTREMES OUTSIDE PERIOD OF RECORD.--Maximum streamflow, 44,100 ft3/s June 23, 1905, gage height $6.50 \mathrm{ft}$, site and datum than in use. No flow at times in 1896, 1902, 1904-05, 1910-11, 1913-14, 1928, all at site downstream, 1931, 1933-41.

Non-exceedance duration hydrograph for Platte River near Duncan, Nebr. (06774000) (1928-2004 period of record)

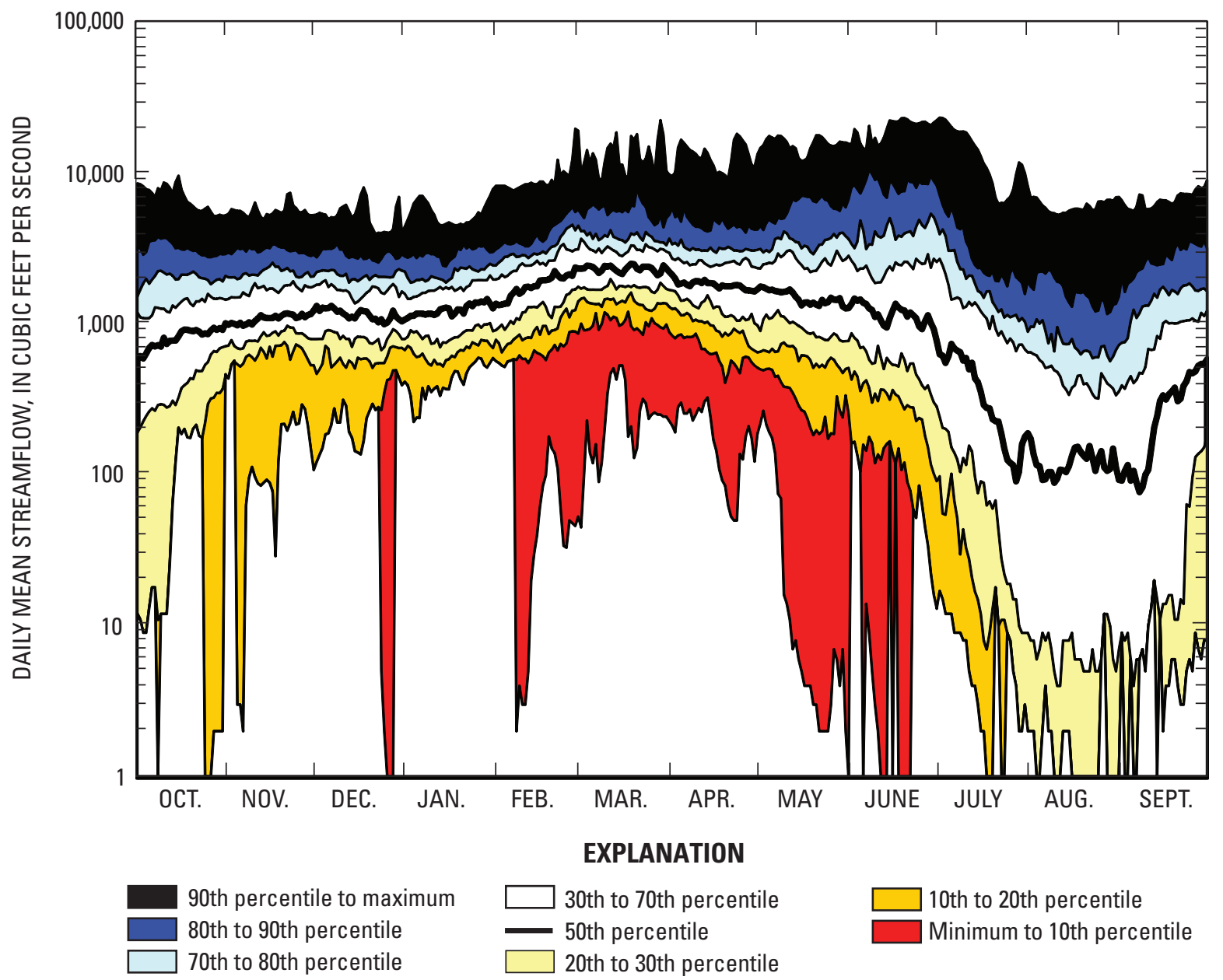


06774000 PLATTE RIVER NEAR DUNCAN, NEBR.

Monthly and annual streamflow, 1942-2005

\begin{tabular}{|c|c|c|c|c|c|}
\hline & $\begin{array}{c}\text { Maximum } \\
\left(\mathrm{ft}^{3} / \mathrm{s}\right)\end{array}$ & $\begin{array}{l}\text { Mini- } \\
\text { mum } \\
\left(\mathrm{ft}^{3} / \mathrm{s}\right)\end{array}$ & $\begin{array}{l}\text { Mean } \\
\left(\mathrm{ft}^{3} / \mathrm{s}\right)\end{array}$ & $\begin{array}{c}\text { Stan- } \\
\text { dard } \\
\text { devia- } \\
\text { tion }\end{array}$ & $\begin{array}{l}\text { Coef- } \\
\text { ficient } \\
\text { of } \\
\text { varia- } \\
\text { tion }\end{array}$ \\
\hline October & 6,673 & 0 & 1,334 & 1,340 & 1.00 \\
\hline November & 5,617 & 0 & 1,491 & 1,089 & 0.73 \\
\hline December & 5,107 & 16 & 1,475 & 961 & 0.65 \\
\hline January & 5,603 & 45 & 1,541 & 953 & 0.62 \\
\hline February & 8,795 & 269 & 2,248 & 1,413 & 0.63 \\
\hline March & 9,531 & 714 & 2,839 & 1,582 & 0.56 \\
\hline April & 13,410 & 362 & 2,437 & 1,943 & 0.80 \\
\hline May & 15,450 & 150 & 2,551 & 2,635 & 1.03 \\
\hline June & 18,320 & 11 & 2,784 & 3,426 & 1.23 \\
\hline July & 12,590 & 0 & 1,371 & 1,969 & 1.44 \\
\hline August & 6,135 & 0 & 629 & 1,012 & 1.61 \\
\hline September & 6,785 & 0 & 922 & 1,289 & 1.40 \\
\hline Annual & 6,652 & 287 & 1,798 & 1,192 & 0.66 \\
\hline
\end{tabular}

Magnitude and probability of annual low flow based on period of record, 1928-2004

\begin{tabular}{|c|c|c|c|c|c|c|}
\hline \multirow{3}{*}{$\begin{array}{l}\text { Period } \\
\text { (con- } \\
\text { secu- } \\
\text { tive } \\
\text { days) }\end{array}$} & \multicolumn{6}{|c|}{$\begin{array}{l}\text { Streamflow, in } \mathrm{ft}^{3} / \mathrm{s} \text {, for indicated recurrence interval } \\
\text { in years, and non-exceedance probability, in percent }\end{array}$} \\
\hline & 2 & 5 & 10 & 20 & 50 & 100 \\
\hline & $50 \%$ & $20 \%$ & $10 \%$ & $5 \%$ & $2 \%$ & $1 \%$ \\
\hline 1 & 38 & 5 & 2 & 1 & 0 & 0 \\
\hline 2 & 37 & 6 & 2 & 1 & 0 & 0 \\
\hline 3 & 39 & 5 & 2 & 1 & 0 & 0 \\
\hline 7 & 40 & 4 & 1 & 0 & 0 & 0 \\
\hline 14 & 57 & 4 & 1 & 0 & 0 & 0 \\
\hline 30 & 81 & 8 & 2 & 1 & 0 & 0 \\
\hline 60 & 200 & 16 & 3 & 0 & 0 & 0 \\
\hline 90 & 361 & 44 & 10 & 2 & 0 & 0 \\
\hline 183 & 1,025 & 165 & 30 & 5 & 0 & 0 \\
\hline \multicolumn{7}{|c|}{ October-November } \\
\hline 1 & 493 & 87 & 26 & 8 & 2 & 1 \\
\hline 2 & 532 & 95 & 28 & 9 & 2 & 1 \\
\hline 3 & 561 & 100 & 29 & 9 & 2 & 1 \\
\hline 7 & 684 & 103 & 25 & 6 & 1 & 0 \\
\hline 14 & 851 & 147 & 39 & 10 & 2 & 0 \\
\hline 30 & 1,008 & 143 & 30 & 6 & 1 & 0 \\
\hline 60 & 1,588 & 282 & 55 & 9 & 1 & 0 \\
\hline
\end{tabular}

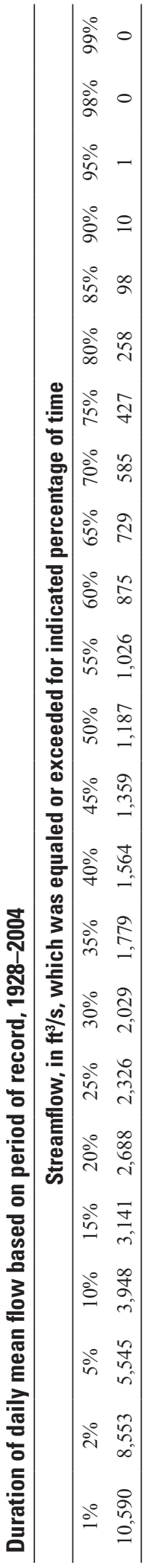




\section{PLATTE RIVER AT NORTH BEND, NEBR.}

LOCATION.--Lat 41 ${ }^{\circ} 27^{\prime} 10^{\prime \prime}$, long 9646'32", in SE 1/4 SE 1/4 sec.7, T.17 N., R.6 D., Dodge County, Hydrologic Unit 10200201, on left bank 80 ft upstream from bridge on State Highway 79, 1 mi south of North Bend, 5 mi downstream from Shell Creek, and at mile 73.0.

DRAINAGE AREA.--70,400 $\mathrm{mi}^{2}$ of which $12,600 \mathrm{mi}^{2}$ probably is noncontributing.

\section{STREAMFLOW RECORDS}

PERIOD OF RECORD.--April 1949 to current year.

GAGE.--Water-stage recorder. Datum of gage is 1,262.32 ft above sea level. Prior to Sept. 12, 1951, nonrecording gage and Sept. 12, 1951 to Sept. 30, 1970 waterstage recorder, at present site at datum $2.00 \mathrm{ft}$ higher. Data collection platform at station.

REMARKS.--Records good except for estimated daily streamflows, which are poor. Natural flow of stream affected by trans-mountain diversions, storage reservoirs, power developments, ground-water withdrawals and diversions for irrigation, and return flow from irrigated areas. EXTREMES FOR PERIOD OF RECORD.--Maximum streamflow, 112,000 ft March 29, 1960 (gage height, 10.04 feet); maximum gage height, 15.55 feet, March 19, 1978 (backwater from ice); minimum daily streamflow, $36 \mathrm{ft}^{3}$ July 29, 1974.

Non-exceedance duration hydrograph for Platte River at North Bend, Nebr. (06796000) (1949-2004 period of record)

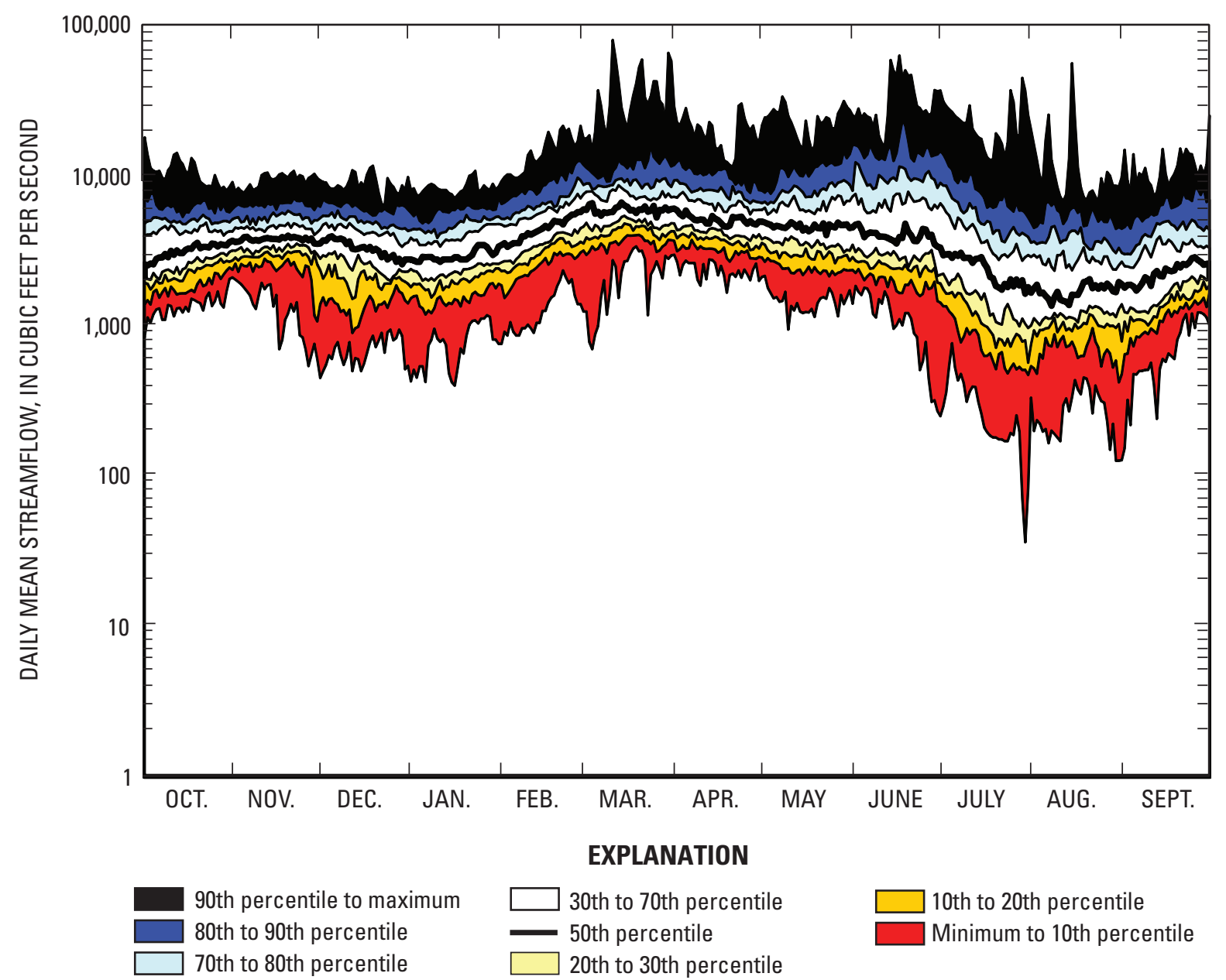


06796000 PLATTE RIVER AT NORTH BEND, NEBR.

Monthly and annual streamflow, 1949-2005

\begin{tabular}{lcrrrr}
\hline & $\begin{array}{c}\text { Maximum } \\
\left(\mathbf{f t t}^{3} / \mathbf{s}\right)\end{array}$ & $\begin{array}{c}\text { Mini- } \\
\text { mum } \\
\left(\mathbf{f t}^{3} / \mathbf{s}\right)\end{array}$ & $\begin{array}{c}\text { Mean } \\
\left(\mathbf{f t}^{3} / \mathbf{s}\right)\end{array}$ & $\begin{array}{c}\text { Stan- } \\
\text { dard } \\
\text { devia- } \\
\text { tion }\end{array}$ & $\begin{array}{c}\text { Coef- } \\
\text { ficient } \\
\text { of } \\
\text { varia- } \\
\text { tion }\end{array}$ \\
\hline October & 10,130 & 1,624 & 3,762 & 1,755 & 0.47 \\
November & 9,462 & 1,938 & 4,130 & 1,453 & 0.35 \\
December & 8,581 & 1,413 & 3,558 & 1,356 & 0.38 \\
January & 7,361 & 932 & 3,377 & 1,419 & 0.42 \\
February & 11,850 & 2,689 & 5,217 & 1,994 & 0.38 \\
March & 16,870 & 3,683 & 7,210 & 2,970 & 0.41 \\
April & 19,400 & 2,672 & 5,982 & 2,738 & 0.46 \\
May & 21,770 & 1,952 & 5,868 & 3,301 & 0.56 \\
June & 25,340 & 1,932 & 6,490 & 4,960 & 0.76 \\
July & 17,070 & 381 & 3,606 & 3,162 & 0.88 \\
August & 8,021 & 442 & 2,461 & 1,697 & 0.69 \\
September & 9,022 & 936 & 3,020 & 1,934 & 0.64 \\
Annual & $\mathbf{1 0 , 8 5 0}$ & $\mathbf{2 , 2 0 1}$ & $\mathbf{4 , 6 3 5}$ & $\mathbf{4 , 6 3 5}$ & $\mathbf{0 . 6 6}$ \\
\hline
\end{tabular}

Magnitude and probability of annual low flow based on period of record, 1949-2004

\begin{tabular}{rrrrrrr}
\hline $\begin{array}{c}\text { Period } \\
\text { (con- } \\
\text { secu- }\end{array}$ & $\begin{array}{c}\text { Streamflow, in ft/s, for indicated recurrence interval, in } \\
\text { years, and non-excedance probability, in percent }\end{array}$ \\
\cline { 2 - 7 } $\begin{array}{c}\text { tive } \\
\text { days) }\end{array}$ & $\mathbf{2}$ & $\mathbf{5}$ & $\mathbf{1 0}$ & $\mathbf{2 0}$ & $\mathbf{5 0}$ & $\mathbf{1 0 0}$ \\
\hline 1 & $\mathbf{2 0 \%}$ & $\mathbf{1 0 \%}$ & $\mathbf{5 \%}$ & $\mathbf{2 \%}$ & $\mathbf{1 \%}$ \\
\hline 2 & 619 & 318 & 209 & 142 & 88 & 63 \\
3 & 716 & 359 & 243 & 171 & 111 & 82 \\
7 & 842 & 474 & 263 & 187 & 123 & 92 \\
14 & 1,087 & 618 & 443 & 253 & 179 & 140 \\
30 & 1,402 & 825 & 610 & 468 & 232 & 181 \\
60 & 1,816 & 1,127 & 865 & 690 & 530 & 277 \\
90 & 2,154 & 1,417 & 1,137 & 947 & 771 & 672 \\
183 & 2,859 & 2,078 & 1,778 & 1,571 & 1,375 & 1,262 \\
\hline \multicolumn{7}{c}{0 ctober-November } \\
\hline 1 & 1,899 & 1,132 & 849 & 663 & 498 & 408 \\
2 & 2,131 & 1,288 & 968 & 756 & 565 & 461 \\
3 & 2,213 & 1,364 & 1,042 & 826 & 631 & 524 \\
7 & 2,467 & 1,706 & 1,416 & 1,218 & 1,032 & 925 \\
14 & 2,804 & 2,010 & 1,701 & 1,487 & 1,283 & 1,165 \\
30 & 3,218 & 2,345 & 1,996 & 1,751 & 1,514 & 1,376 \\
60 & 3,619 & 2,730 & 2,387 & 2,149 & 1,923 & 1,792 \\
\hline \multicolumn{7}{c}{}
\end{tabular}

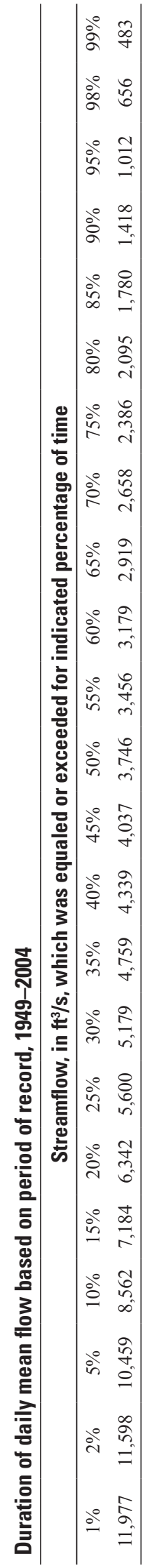




\section{PLATTE RIVER NEAR LESHARA, NEBR.}

LOCATION.--Lat 41 $19^{\prime} 12^{\prime \prime}$, long 96²4'14", in NW 1/4 NW 1/4 sec.34, T.16 N., R.9 E., Douglas County, Hydrologic Unit 10200202, on left bank $25 \mathrm{ft}$ downstream from bridge on Nebraska Highway 64, 1.0 mi southeast of Leshara, NE.

DRAINAGE AREA.-

\section{STREAMFLOW RECORDS}

PERIOD OF RECORD.--June 1994 to current year.

GAGE.--Water-stage recorder. Datum of gage is 1,143.86 ft above sea level. Data collection platform at station.

REMARKS.--Records good except for estimated daily streamflows, which are poor.

Non-exceedance duration hydrograph for Platte River near Leshara, Nebr. (06796500) (1994-2004 period of record)

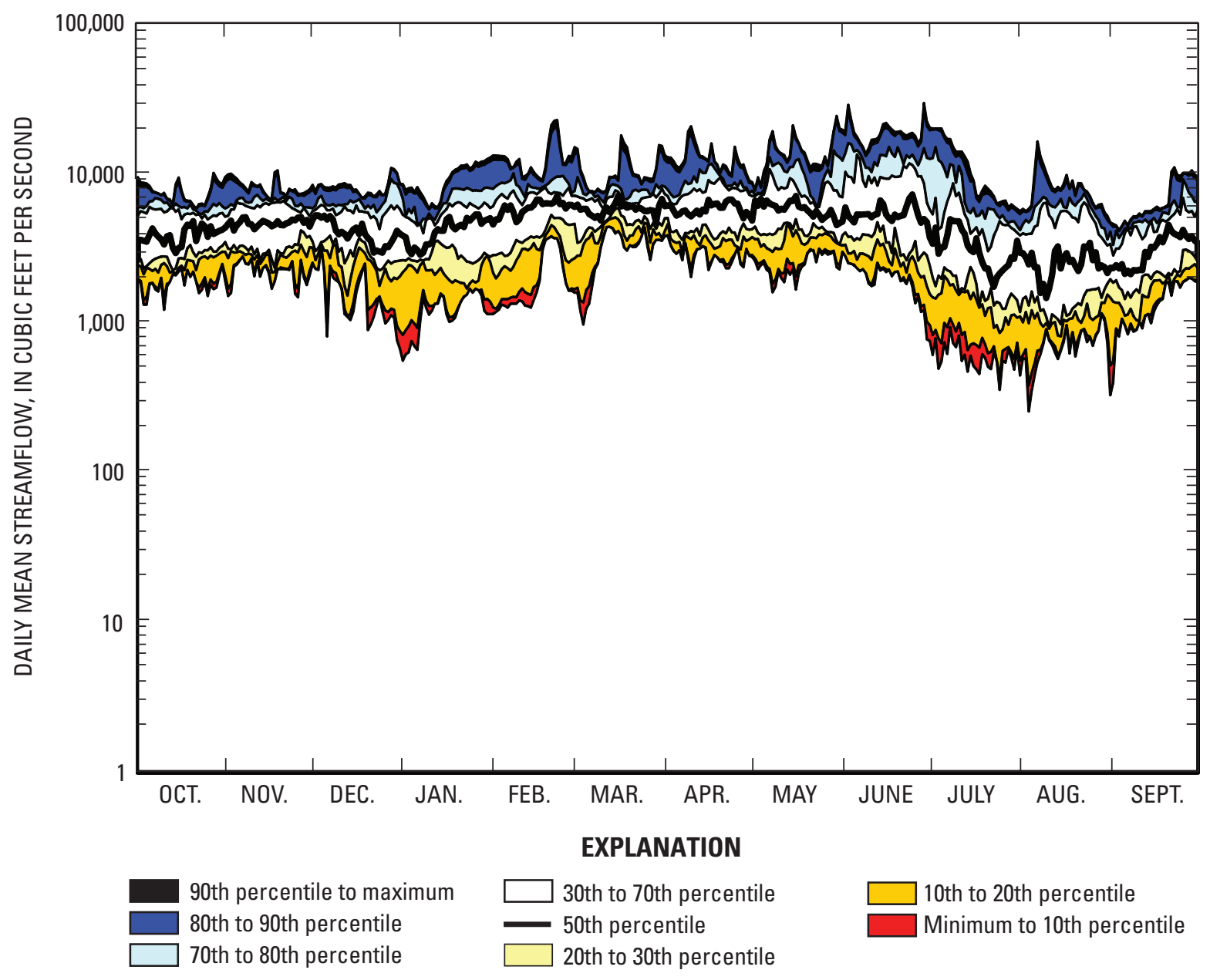


06796500 PLATTE RIVER NEAR LESHARA, NEBR.

Monthly and annual streamflow, 1994-2004

\begin{tabular}{lccccc}
\hline & $\begin{array}{c}\text { Maximum } \\
\left(\mathbf{f t t}^{\mathbf{3}} \mathbf{s}\right)\end{array}$ & $\begin{array}{c}\text { Mini- } \\
\text { mum } \\
\left(\mathbf{f t t}^{3} / \mathbf{s}\right)\end{array}$ & $\begin{array}{c}\text { Mean } \\
\left(\mathbf{f t}^{3} / \mathbf{s}\right)\end{array}$ & $\begin{array}{c}\text { Stan- } \\
\text { dard } \\
\text { devia- } \\
\text { tion }\end{array}$ & $\begin{array}{c}\text { Coef- } \\
\text { ficient } \\
\text { of } \\
\text { varia- } \\
\text { tion }\end{array}$ \\
\hline October & 6,733 & 1,924 & 4,282 & 1,726 & 0.40 \\
November & 7,784 & 2,324 & 4,798 & 1,754 & 0.37 \\
December & 6,762 & 1,984 & 4,372 & 1,643 & 0.38 \\
January & 7,552 & 1,276 & 3,957 & 1,914 & 0.48 \\
February & 10,040 & 2,984 & 5,741 & 2,335 & 0.41 \\
March & 7,960 & 4,079 & 5,999 & 1,374 & 0.23 \\
April & 11,300 & 3,054 & 6,348 & 2,681 & 0.42 \\
May & 10,650 & 3,180 & 6,585 & 2,487 & 0.38 \\
June & 17,460 & 2,446 & 7,444 & 4,991 & 0.67 \\
July & 10,540 & 595 & 4,089 & 2,901 & 0.71 \\
August & 7,163 & 948 & 3,171 & 2,126 & 0.67 \\
September & 6,793 & 1,710 & 3,390 & 1,654 & 0.49 \\
Annual & $\mathbf{7 , 4 4 4}$ & $\mathbf{2 , 7 0 3}$ & $\mathbf{4 , 9 9 4}$ & $\mathbf{1 , 8 7 8}$ & $\mathbf{0 . 3 8}$ \\
\hline
\end{tabular}

Magnitude and probability of annual low flow based on period of record, 1994-2004

\begin{tabular}{|c|c|c|c|c|c|c|}
\hline \multirow{3}{*}{$\begin{array}{c}\text { Period } \\
\text { (con- } \\
\text { secu- } \\
\text { tive } \\
\text { days) }\end{array}$} & \multicolumn{6}{|c|}{$\begin{array}{l}\text { Streamflow, in } \mathrm{ft}^{3} / \mathrm{s} \text {, for indicated recurrence interval, in } \\
\text { years, and non-exceedance probability, in percent }\end{array}$} \\
\hline & 2 & 5 & 10 & 20 & 50 & 100 \\
\hline & $50 \%$ & $20 \%$ & $10 \%$ & $5 \%$ & $2 \%$ & $1 \%$ \\
\hline 1 & 904 & 484 & 339 & 248 & 172 & 133 \\
\hline 2 & 966 & 524 & 377 & 285 & 207 & 166 \\
\hline 3 & 1,047 & 602 & 449 & 352 & 267 & 222 \\
\hline 7 & 1,233 & 733 & 562 & 452 & 356 & 303 \\
\hline 14 & 1,552 & 897 & 667 & 520 & 391 & 322 \\
\hline 30 & 1,947 & 1,080 & 773 & 577 & 410 & 323 \\
\hline 60 & 2,444 & 1,324 & 930 & 682 & 472 & 366 \\
\hline 90 & 2,884 & 1,699 & 1,259 & 971 & 715 & 579 \\
\hline 183 & 3,550 & 2,350 & 1,871 & 1,539 & 1,227 & 1,051 \\
\hline \multicolumn{7}{|c|}{ October-November } \\
\hline 1 & 2,907 & 1,920 & 1,519 & 1,241 & 979 & 830 \\
\hline 2 & 3,065 & 2,035 & 1,616 & 1,324 & 1,048 & 891 \\
\hline 3 & 3,140 & 2,114 & 1,701 & 1,414 & 1,141 & 985 \\
\hline 7 & 3,391 & 2,335 & 1,903 & 1,599 & 1,307 & 1,138 \\
\hline 14 & 3,571 & 2,496 & 2,055 & 1,743 & 1,443 & 1,269 \\
\hline 30 & 3,912 & 2,720 & 2,225 & 1,874 & 1,535 & 1,339 \\
\hline 60 & 4,282 & 3,007 & 2,473 & 2,094 & 1,725 & 1,511 \\
\hline
\end{tabular}

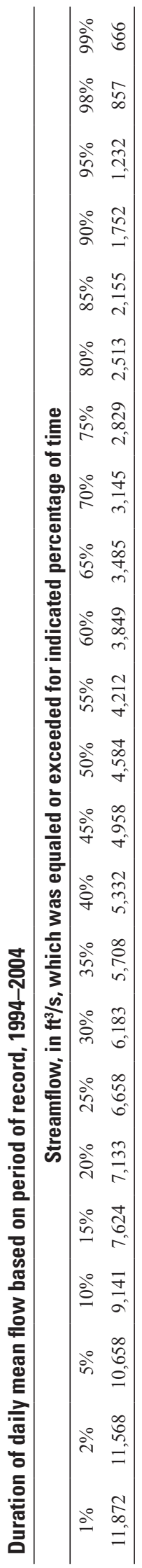


06796973 ELKHORN RIVER NEAR ATKINSON, NEBR.

LOCATION.--Lat 42²9'12", long 9854'43", Holt County, Hydrologic Unit 10220001

DRAINAGE AREA.--586 $\mathrm{mi}^{2}$.

\section{STREAMFLOW RECORDS}

PERIOD OF RECORD.--October 1982 to current year.

GAGE.-- Datum of gage is $2042 \mathrm{ft}$ above sea level.

REMARKS.--Records good except for estimated daily streamflows, which are poor.

Non-exceedance duration hydrograph for Elkhorn River near Atkinson, Nebr. (06796973) (1982-2004 period of record)

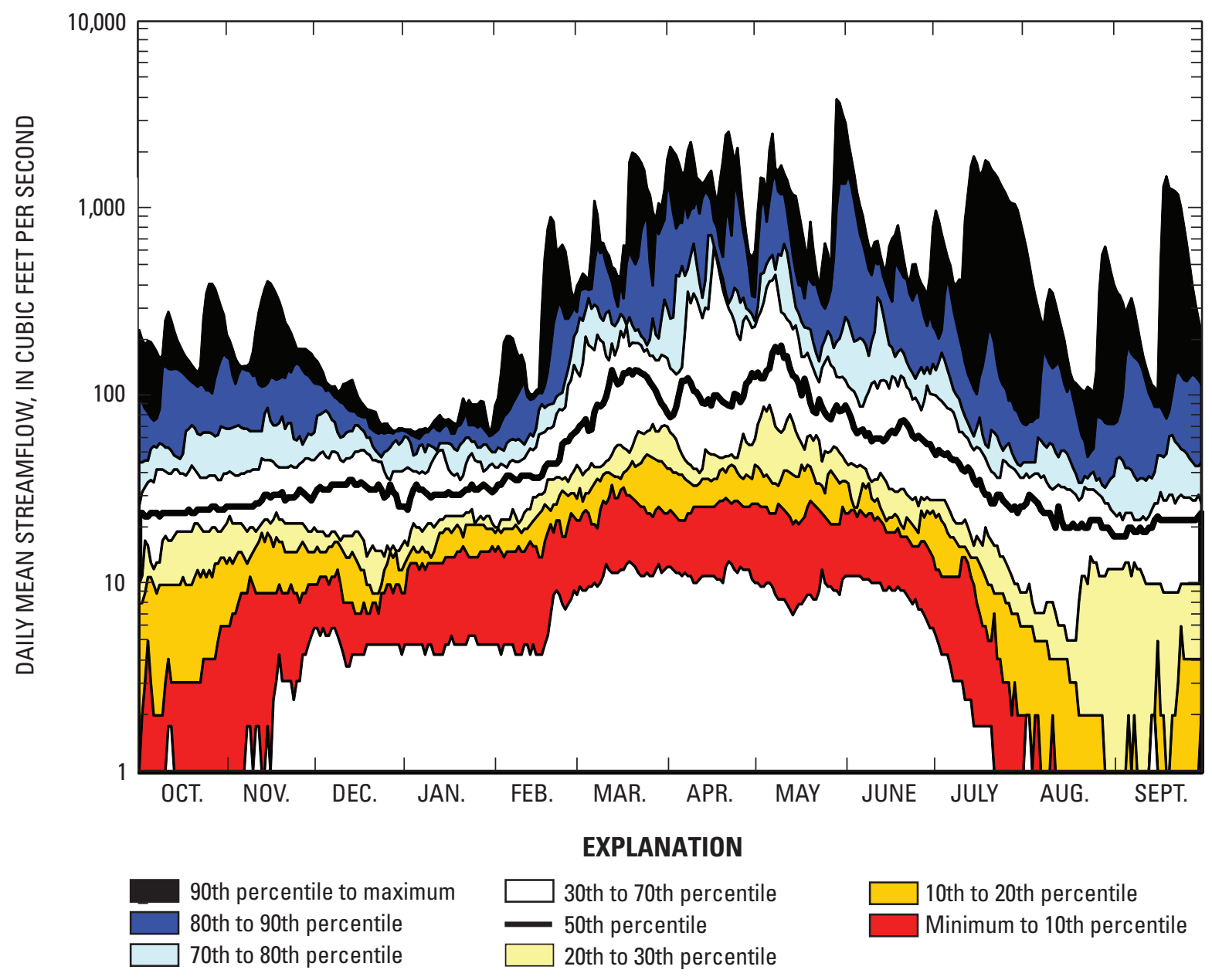


06796973 ELKHORN RIVER NEAR ATKINSON, NEBR.

Monthly and annual streamflow, 1982-2004

\begin{tabular}{lrrrrr}
\hline & $\begin{array}{c}\text { Maximum } \\
\left(\mathbf{f t}^{3} / \mathbf{s}\right)\end{array}$ & $\begin{array}{c}\text { Mini- } \\
\mathbf{m u m} \\
\left(\mathbf{f t}^{3} / \mathbf{s}\right)\end{array}$ & $\begin{array}{c}\text { Mean } \\
\left(\mathbf{f t}^{3} / \mathbf{s}\right)\end{array}$ & $\begin{array}{c}\text { Stan- } \\
\text { dard } \\
\text { devia- } \\
\text { tion }\end{array}$ & $\begin{array}{c}\text { Coef- } \\
\text { ficient } \\
\text { of } \\
\text { varia- } \\
\text { tion }\end{array}$ \\
\hline October & 213 & 1 & 42 & 49 & 1.19 \\
November & 220 & 3 & 48 & 52 & 1.07 \\
December & 92 & 7 & 38 & 26 & 0.68 \\
January & 74 & 7 & 35 & 19 & 0.53 \\
February & 288 & 14 & 68 & 63 & 0.92 \\
March & 760 & 23 & 186 & 182 & 0.98 \\
April & 1,010 & 21 & 274 & 294 & 1.07 \\
May & 1,332 & 17 & 241 & 281 & 1.17 \\
June & 566 & 19 & 136 & 160 & 1.17 \\
July & 1,040 & 3 & 95 & 218 & 2.30 \\
August & 215 & 1 & 38 & 54 & 1.44 \\
September & 448 & 1 & 50 & 96 & 1.92 \\
Annual & $\mathbf{3 0 3}$ & $\mathbf{1 1}$ & $\mathbf{1 0 5}$ & $\mathbf{7 7}$ & $\mathbf{0 . 7 3}$ \\
\hline
\end{tabular}

Magnitude and probability of annual low flow based on period of record, 1982-2004

\begin{tabular}{|c|c|c|c|c|c|c|}
\hline \multirow{3}{*}{$\begin{array}{c}\text { Period } \\
\text { (con- } \\
\text { secu- } \\
\text { tive } \\
\text { days) }\end{array}$} & \multicolumn{6}{|c|}{$\begin{array}{l}\text { Streamflow, in } \mathrm{ft}^{3} / \mathrm{s} \text {, for indicated recurrence interval, } \\
\text { in years, and non-exceedance probability, in percent }\end{array}$} \\
\hline & 2 & 5 & 10 & 20 & 50 & 100 \\
\hline & $50 \%$ & $20 \%$ & $10 \%$ & $5 \%$ & $2 \%$ & $1 \%$ \\
\hline 1 & 9 & 2 & 1 & 0 & 0 & 0 \\
\hline 2 & 9 & 2 & 1 & 0 & 0 & 0 \\
\hline 3 & 10 & 2 & 1 & 0 & 0 & 0 \\
\hline 7 & 10 & 3 & 1 & 0 & 0 & 0 \\
\hline 14 & 11 & 3 & 1 & 1 & 0 & 0 \\
\hline 30 & 13 & 4 & 1 & 1 & 0 & 0 \\
\hline 60 & 17 & 5 & 2 & 1 & 0 & 0 \\
\hline 90 & 20 & 6 & 3 & 1 & 1 & 0 \\
\hline 183 & 25 & 11 & 7 & 4 & 3 & 2 \\
\hline \multicolumn{7}{|c|}{ October-November } \\
\hline 1 & 18 & 5 & 2 & 1 & 0 & 0 \\
\hline 2 & 18 & 6 & 3 & 1 & 1 & 0 \\
\hline 3 & 19 & 6 & 3 & 1 & 1 & 0 \\
\hline 7 & 21 & 7 & 3 & 2 & 1 & 0 \\
\hline 14 & 22 & 7 & 4 & 2 & 1 & 0 \\
\hline 30 & 25 & 9 & 4 & 2 & 1 & 1 \\
\hline 60 & 28 & 11 & 7 & 4 & 3 & 2 \\
\hline
\end{tabular}

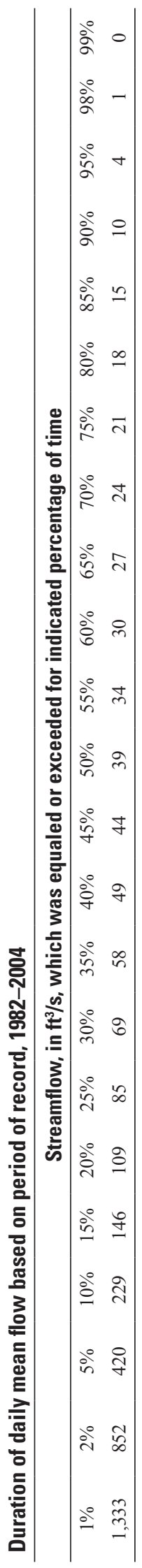




\section{ELKHORN RIVER AT EWING, NEBR.}

LOCATION.--Lat $42^{\circ} 16^{\prime} 07^{\prime \prime}$, long 98 $20^{\circ} 22^{\prime \prime}$, in NW 1/4 SW 1/4 sec.35, T.27 N., R.9 W., Holt County, Hydrologic Unit 10220001, on left bank $10 \mathrm{ft}$ downstream from bridge on State Highway L-45B, 0.8 mi north of Ewing, 1.5 mi upstream from South Fork Elkhorn River, and at mile 199.

DRAINAGE AREA.--1,400 $\mathrm{mi}^{2}$, of which $660 \mathrm{mi}^{2}$ probably is noncontributing.

\section{STREAMFLOW RECORDS}

PERIOD OF RECORD.--August 1947 to current year.

GAGE.--Water-stage recorder. Datum of gage is 1,836.24 ft above sea level, levels by Nebraska Department of Roads. From August 1, 1947 to October 22, 1952 at site $300 \mathrm{ft}$ downstream at same datum. From October 23, 1952 to Sept. 30, 1996 at site $500 \mathrm{ft}$ downstream at same datum. Data collection platform at station.

REMARKS.--Records good except for estimated daily streamflows, which are poor.

Non-exceedance duration hydrograph for Elkhorn River at Ewing, Nebr. (06797500) (1947-2004 period of record)

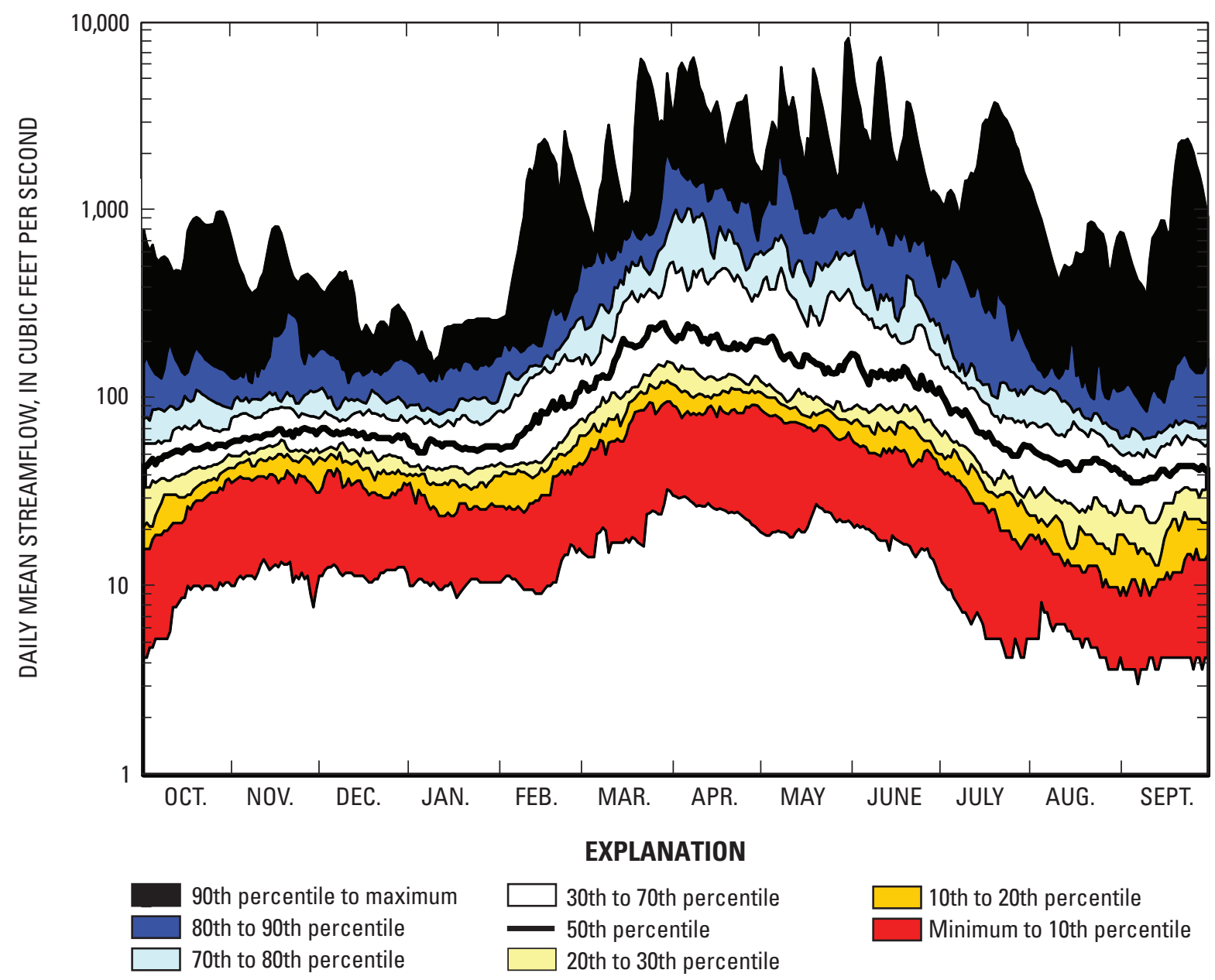


06797500 ELKHORN RIVER AT EWING, NEBR.

Monthly and annual streamflow, 1947-2004

\begin{tabular}{lccrcc}
\hline & $\begin{array}{c}\text { Maximum } \\
\left(\mathbf{f t}^{3} / \mathbf{s}\right)\end{array}$ & $\begin{array}{c}\text { Mini- } \\
\mathbf{m u m} \\
\left(\mathbf{f t}^{3} / \mathbf{s}\right)\end{array}$ & $\begin{array}{c}\text { Mean } \\
\left(\mathbf{f t t}^{3} / \mathbf{s}\right)\end{array}$ & $\begin{array}{c}\text { Stan- } \\
\text { dard } \\
\text { devia- } \\
\text { tion }\end{array}$ & $\begin{array}{c}\text { Coef- } \\
\text { ficient } \\
\text { of } \\
\text { varia- } \\
\text { tion }\end{array}$ \\
\hline October & 671 & 15 & 86 & 117 & 1.36 \\
November & 443 & 23 & 92 & 85 & 0.92 \\
December & 250 & 24 & 80 & 52 & 0.65 \\
January & 226 & 19 & 70 & 44 & 0.62 \\
February & 1,172 & 26 & 137 & 175 & 1.27 \\
March & 2,144 & 61 & 344 & 349 & 1.01 \\
April & 2,081 & 60 & 488 & 536 & 1.10 \\
May & 2,243 & 52 & 408 & 435 & 1.07 \\
June & 2,690 & 46 & 305 & 426 & 1.40 \\
July & 1,993 & 11 & 159 & 292 & 1.83 \\
August & 444 & 11 & 74 & 89 & 1.20 \\
September & 882 & 6 & 75 & 132 & 1.75 \\
Annual & $\mathbf{5 4 3}$ & $\mathbf{4 3}$ & $\mathbf{1 9 4}$ & $\mathbf{1 4 3}$ & $\mathbf{0 . 7 4}$ \\
\hline
\end{tabular}

Magnitude and probability of annual low flow based on period of record, 1947-2004

\begin{tabular}{|c|c|c|c|c|c|c|}
\hline \multirow{3}{*}{$\begin{array}{c}\text { Period } \\
\text { (con- } \\
\text { secu- } \\
\text { tive } \\
\text { days) }\end{array}$} & \multicolumn{6}{|c|}{$\begin{array}{l}\text { Streamflow, in } \mathrm{ft}^{3} / \mathrm{s} \text {, for indicated recurrence interval, } \\
\text { in years, and non-exceedance probability, in percent }\end{array}$} \\
\hline & 2 & 5 & 10 & 20 & 50 & 100 \\
\hline & $50 \%$ & $20 \%$ & $10 \%$ & $5 \%$ & $2 \%$ & $1 \%$ \\
\hline 1 & 904 & 484 & 339 & 248 & 172 & 133 \\
\hline 2 & 966 & 524 & 377 & 285 & 207 & 166 \\
\hline 3 & 1,047 & 602 & 449 & 352 & 267 & 222 \\
\hline 7 & 1,233 & 733 & 562 & 452 & 356 & 303 \\
\hline 14 & 1,552 & 897 & 667 & 520 & 391 & 322 \\
\hline 30 & 1,947 & 1,080 & 773 & 577 & 410 & 323 \\
\hline 60 & 2,444 & 1,324 & 930 & 682 & 472 & 366 \\
\hline 90 & 2,884 & 1,699 & 1,259 & 971 & 715 & 579 \\
\hline 183 & 3,550 & 2,350 & 1,871 & 1,539 & 1,227 & 1,051 \\
\hline \multicolumn{7}{|c|}{ October-November } \\
\hline 1 & 36 & 20 & 15 & 12 & 9 & 8 \\
\hline 2 & 37 & 21 & 15 & 12 & 9 & 8 \\
\hline 3 & 38 & 21 & 16 & 12 & 10 & 8 \\
\hline 7 & 42 & 24 & 18 & 14 & 11 & 9 \\
\hline 14 & 45 & 26 & 20 & 17 & 14 & 12 \\
\hline 30 & 50 & 32 & 26 & 23 & 20 & 19 \\
\hline 60 & 59 & 38 & 33 & 29 & 27 & 26 \\
\hline
\end{tabular}

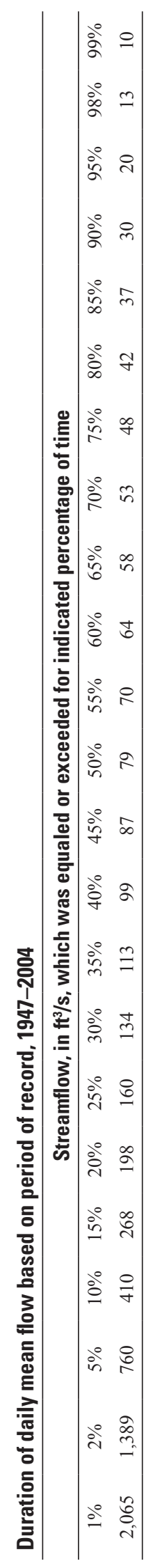




\section{SOUTH FORK ELKHORN RIVER AT EWING, NEBR.}

LOCATION.--Lat $42^{\circ} 14^{\prime} 29^{\prime \prime}$, long $98^{\circ} 23^{\prime} 54^{\prime \prime}$, in SE 1/4 NE 1/4 sec.7, T.26 N., R.9 W., Holt County, Hydrologic Unit 10220001, on right bank 7 feet downstream and 80 feet landward from bridge on county highway, 3.1 mi southwest of U.S. highway 275 bridge at Ewing, and 5.5 mi upstream from mouth.

DRAINAGE AREA.--314 $\mathrm{mi}^{2}$, approximately, of which about $190 \mathrm{mi}^{2}$ contributes directly to surface runoff

$$
\text { STREAMFLOW RECORDS }
$$

PERIOD OF RECORD.--October 1947 to September 1953, August 1960 to September 1972, October 1977 to present.

Non-exceedance duration hydrograph for South Fork Elkhorn River at Ewing, Nebr. (06798000) (1947-2004 period of record)

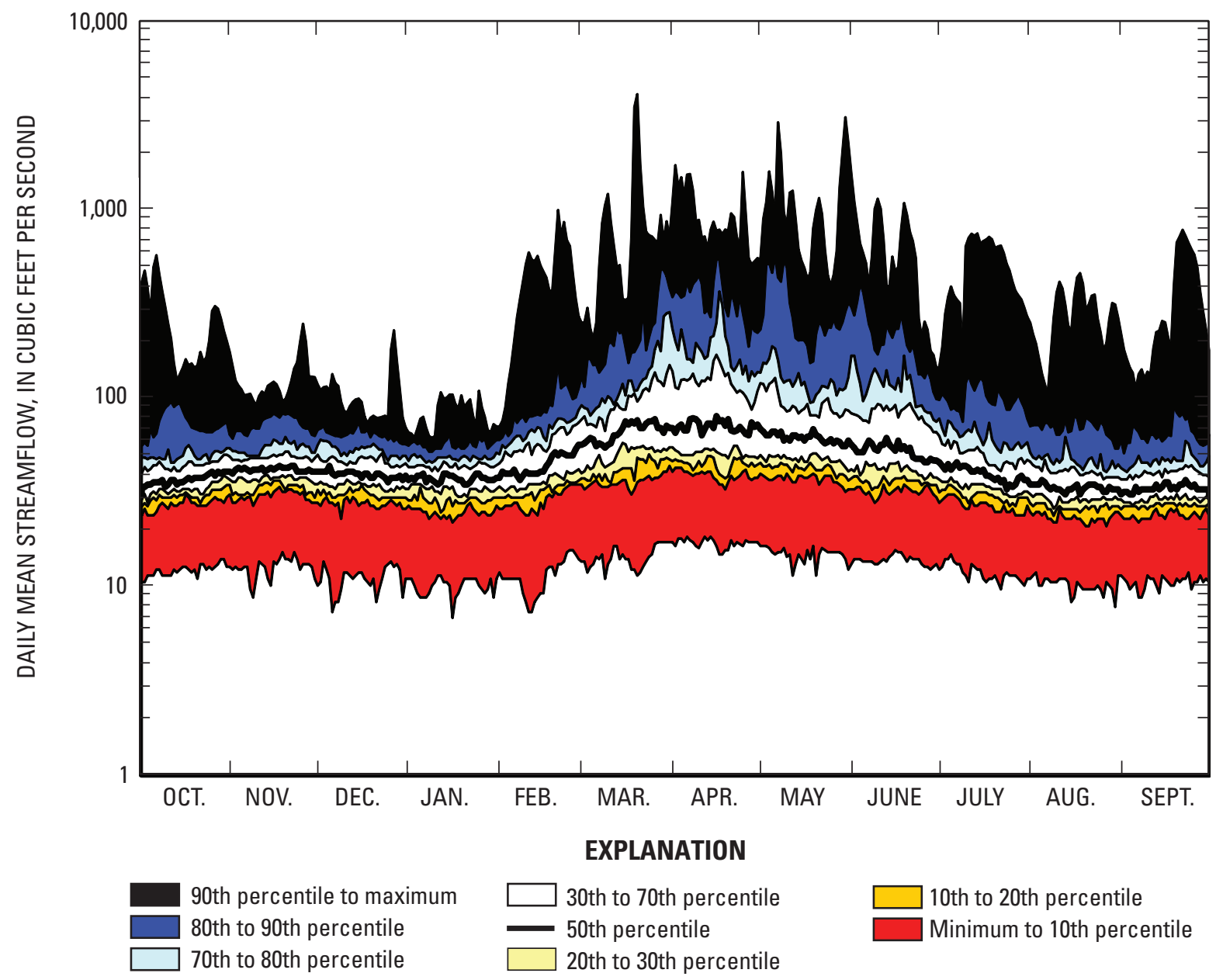


Monthly and annual streamflow, 1948-2004

\begin{tabular}{lccccc}
\hline & $\begin{array}{c}\text { Maximum } \\
\left(\mathbf{f t}^{3} \mathbf{s}\right)\end{array}$ & $\begin{array}{c}\text { Mini- } \\
\mathbf{m u m} \\
\left(\mathbf{f t}^{3} \mathbf{s} \mathbf{s}\right)\end{array}$ & $\begin{array}{c}\text { Mean } \\
\left(\mathbf{f t}^{3} \mathbf{s}\right)\end{array}$ & $\begin{array}{c}\text { Stan- } \\
\text { dard } \\
\text { devia- } \\
\text { tion }\end{array}$ & $\begin{array}{c}\text { Coef- } \\
\text { ficient } \\
\text { of } \\
\text { varia- } \\
\text { tion }\end{array}$ \\
\hline October & 203 & 31 & 52 & 46 & 0.88 \\
November & 87 & 30 & 43 & 13 & 0.29 \\
December & 61 & 25 & 37 & 8 & 0.22 \\
January & 47 & 21 & 34 & 8 & 0.22 \\
February & 306 & 26 & 57 & 63 & 1.10 \\
March & 411 & 36 & 116 & 101 & 0.86 \\
April & 449 & 42 & 113 & 100 & 0.88 \\
May & 323 & 37 & 96 & 79 & 0.83 \\
June & 421 & 36 & 99 & 95 & 0.96 \\
July & 275 & 28 & 56 & 56 & 1.00 \\
August & 159 & 23 & 46 & 36 & 0.78 \\
September & 134 & 24 & 44 & 31 & 0.70 \\
Annual & $\mathbf{1 2 7}$ & $\mathbf{3 5}$ & $\mathbf{6 6}$ & $\mathbf{3 1}$ & $\mathbf{0 . 4 8}$ \\
\hline
\end{tabular}

Magnitude and probability of annual low flow based on period of record, 1948-2004

\begin{tabular}{|c|c|c|c|c|c|c|}
\hline \multirow{3}{*}{$\begin{array}{l}\text { Period } \\
\text { (con- } \\
\text { secu- } \\
\text { tive } \\
\text { days) }\end{array}$} & \multicolumn{6}{|c|}{$\begin{array}{l}\text { Streamflow, in } \mathrm{ft}^{3} / \mathrm{s} \text {, for indicated recurrence interval, } \\
\text { in years, and non-exceedance probability, in percent }\end{array}$} \\
\hline & 2 & 5 & 10 & 20 & 50 & 100 \\
\hline & $50 \%$ & $20 \%$ & $10 \%$ & $5 \%$ & $2 \%$ & $1 \%$ \\
\hline 1 & 22 & 17 & 14 & 13 & 11 & 10 \\
\hline 2 & 22 & 18 & 15 & 14 & 12 & 11 \\
\hline 3 & 23 & 18 & 16 & 15 & 13 & 12 \\
\hline 7 & 25 & 20 & 18 & 17 & 15 & 14 \\
\hline 14 & 26 & 22 & 20 & 18 & 17 & 16 \\
\hline 30 & 29 & 24 & 22 & 21 & 19 & 18 \\
\hline 60 & 31 & 26 & 24 & 23 & 22 & 21 \\
\hline 90 & 32 & 28 & 26 & 25 & 25 & 24 \\
\hline 183 & 36 & 31 & 30 & 29 & 29 & 29 \\
\hline \multicolumn{7}{|c|}{ October-November } \\
\hline 1 & 27 & 21 & 19 & 17 & 16 & 15 \\
\hline 2 & 28 & 22 & 20 & 19 & 17 & 17 \\
\hline 3 & 29 & 23 & 21 & 20 & 19 & 18 \\
\hline 7 & 31 & 26 & 24 & 23 & 22 & 21 \\
\hline 14 & 34 & 28 & 26 & 25 & 24 & 24 \\
\hline 30 & 36 & 30 & 29 & 28 & 27 & 27 \\
\hline 60 & 40 & 33 & 31 & 30 & 29 & 29 \\
\hline
\end{tabular}

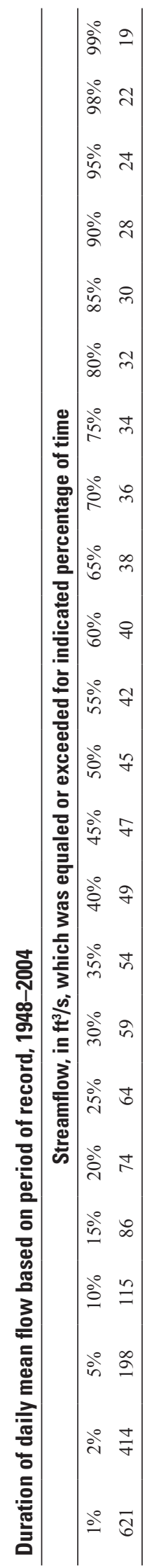


06798500 ELKHORN RIVER AT NELIGH, NEBR.

LOCATION.--Lat 4207'23", long 9802'37", in NW 1/4 SW 1/4 sec.20, T.25 N., R.6 W., Antelope County, Hydrologic Unit 10220001, at State Highway 14 bridge, 0.9 mi southwest of Neligh, 1.2 mi downstream from Hail Creek, and 1.5 mi downstream from Antelope Creek.

DRAINAGE AREA.--2,200 mi², of which 1,000 $\mathrm{mi}^{2}$ probably is noncontributing.

Non-exceedance duration hydrograph for Elkhorn River at Neligh, Nebr. (06798500) (1930-2004 period of record)

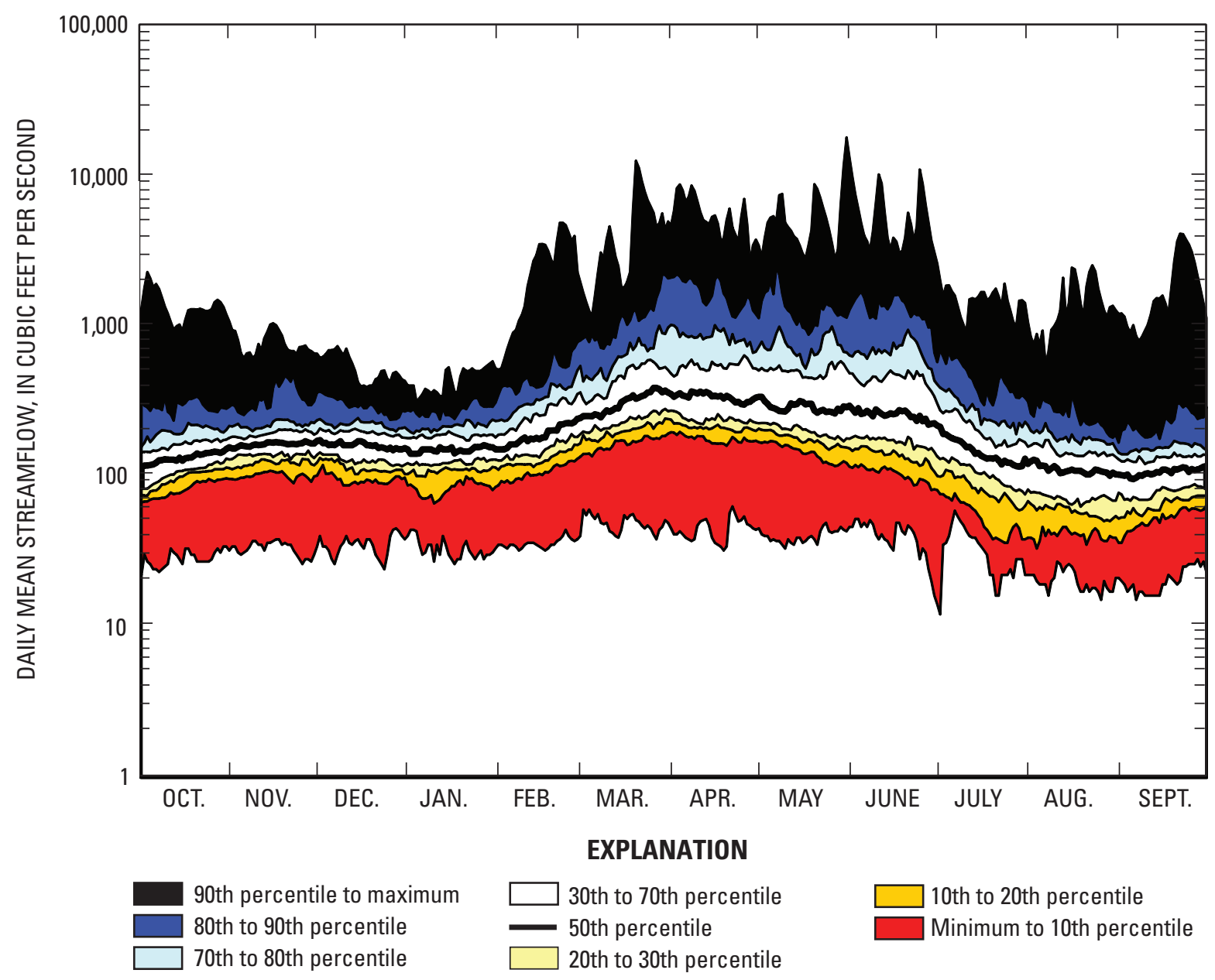


06798500 ELKHORN RIVER AT NELIGH, NEBR.

Monthly and annual streamflow, 1931-2004

\begin{tabular}{lrrrrr}
\hline & $\begin{array}{c}\text { Maximum } \\
\left(\mathbf{f t}^{3} / \mathbf{s}\right)\end{array}$ & $\begin{array}{c}\text { Mini- } \\
\mathbf{m u m} \\
\left(\mathbf{f t}^{3} / \mathbf{s}\right)\end{array}$ & $\begin{array}{c}\text { Mean } \\
\left(\mathbf{f t}^{3} \mathbf{s}\right)\end{array}$ & $\begin{array}{c}\text { Stan- } \\
\text { dard } \\
\text { devia- } \\
\text { tion }\end{array}$ & $\begin{array}{c}\text { Coef- } \\
\text { ficient } \\
\text { of } \\
\text { varia- } \\
\text { tion }\end{array}$ \\
\hline October & 1,189 & 71 & 179 & 192 & 1.07 \\
November & 616 & 85 & 176 & 88 & 0.50 \\
December & 479 & 86 & 163 & 63 & 0.39 \\
January & 324 & 67 & 152 & 52 & 0.34 \\
February & 1,617 & 96 & 230 & 227 & 0.99 \\
March & 2,008 & 183 & 432 & 369 & 0.85 \\
April & 3,141 & 176 & 520 & 515 & 0.99 \\
May & 2,589 & 126 & 490 & 483 & 0.99 \\
June & 3,387 & 86 & 544 & 675 & 1.24 \\
July & 1,043 & 45 & 220 & 238 & 1.08 \\
August & 1,060 & 28 & 150 & 176 & 1.17 \\
September & 877 & 30 & 132 & 132 & 1.00 \\
Annual & $\mathbf{9 3 5}$ & $\mathbf{1 0 8}$ & $\mathbf{2 8 2}$ & $\mathbf{1 8 1}$ & $\mathbf{0 . 6 4}$ \\
\hline
\end{tabular}

Magnitude and probability of annual low flow based on period of record, 1931-2004

\begin{tabular}{|c|c|c|c|c|c|c|}
\hline \multirow{3}{*}{$\begin{array}{c}\text { Period } \\
\text { (con- } \\
\text { secu- } \\
\text { tive } \\
\text { days) } \\
\end{array}$} & \multicolumn{6}{|c|}{$\begin{array}{l}\text { Streamflow, in } \mathrm{ft}^{3} / \mathrm{s} \text {, for indicated recurrence interval, } \\
\text { in years, and non-exceedance probability, in percent }\end{array}$} \\
\hline & 2 & 5 & 10 & 20 & 50 & 100 \\
\hline & $50 \%$ & $20 \%$ & $10 \%$ & $5 \%$ & $2 \%$ & $1 \%$ \\
\hline 1 & 61 & 35 & 26 & 19 & 14 & 11 \\
\hline 2 & 63 & 36 & 26 & 20 & 14 & 12 \\
\hline 3 & 64 & 37 & 27 & 21 & 15 & 12 \\
\hline 7 & 68 & 41 & 30 & 23 & 17 & 14 \\
\hline 14 & 75 & 46 & 35 & 27 & 21 & 17 \\
\hline 30 & 84 & 52 & 40 & 31 & 24 & 20 \\
\hline 60 & 96 & 60 & 47 & 38 & 29 & 25 \\
\hline 90 & 106 & 68 & 53 & 44 & 35 & 30 \\
\hline 183 & 128 & 87 & 73 & 64 & 56 & 51 \\
\hline \multicolumn{7}{|c|}{ October-November } \\
\hline 1 & 99 & 64 & 50 & 40 & 32 & 27 \\
\hline 2 & 102 & 66 & 52 & 42 & 33 & 29 \\
\hline 3 & 105 & 68 & 53 & 43 & 34 & 29 \\
\hline 7 & 113 & 73 & 58 & 48 & 39 & 33 \\
\hline 14 & 122 & 79 & 64 & 53 & 44 & 39 \\
\hline 30 & 134 & 89 & 73 & 63 & 53 & 48 \\
\hline 60 & 151 & 99 & 82 & 71 & 61 & 56 \\
\hline
\end{tabular}

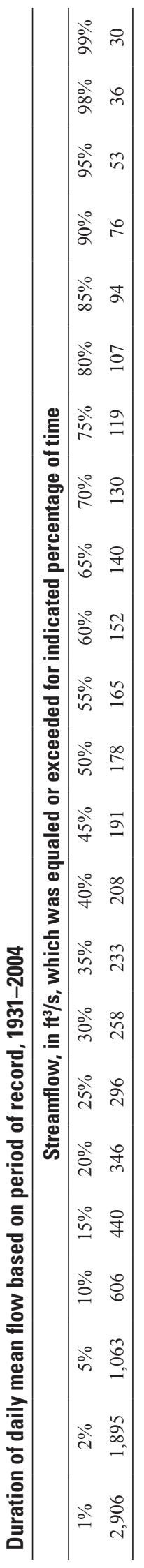


06799000 ELKHORN RIVER AT NORFOLK, NEBR.

LOCATION.--Lat 42 ${ }^{\circ} 00^{\prime} 14^{\prime \prime}$, long 97³1'22", in SW 1/4 SW 1/4 sec.34, T.24 N., R.1 W., Madison County, Hydrologic Unit 10220001, on left bank $200 \mathrm{ft}$ downstream from U.S. Highway 81 bridge, 1 mi south of intersection of U.S. Highways 81 and 275, and 3.6 mi upstream from North Fork Elkhorn River, and at mile 129.

DRAINAGE AREA.--2,790 $\mathrm{mi}^{2}$ of which $1,000 \mathrm{mi}^{2}$ probably is noncontributing.

\section{STREAMFLOW RECORDS}

REVISED RECORDS.--WSP 1390: 1898-1900. WSP 1730: Drainage area.

PERIOD OF RECORD.--July 1896 to November 1903 (no winter records), October 1945 to current year. Gage height records collected at site $200 \mathrm{ft}$ upstream from May 10, 1941 to Sept. 26, 1945 are contained in reports of U.S. Weather Bureau. Published as "near Norfolk" from 0ctober 1957 to September 1977.

GAGE.--Water-stage recorder. Datum of gage is 1,500.95 ft above sea level. See WSP 1918 for history of changes prior to Aug. 30, 1958. Aug. 30,1958 , to July 27,1978 , water-stage recorder at site 3.2 mi upstream at datum $19.88 \mathrm{ft}$ higher and July 28, 1978 to Mar. 18, 1987, present site at datum $4.00 \mathrm{ft}$ higher. Mar. 19, 1987, to Mar. 31, 1995, present site at datum $2.00 \mathrm{ft}$ higher. Data collection platform at station.

REMARKS.--Records good except for estimated daily streamflows, which are poor.

Non-exceedance duration hydrograph for Elkhorn River at Norfolk, Nebr. (06799000) (1896-2004 period of record)

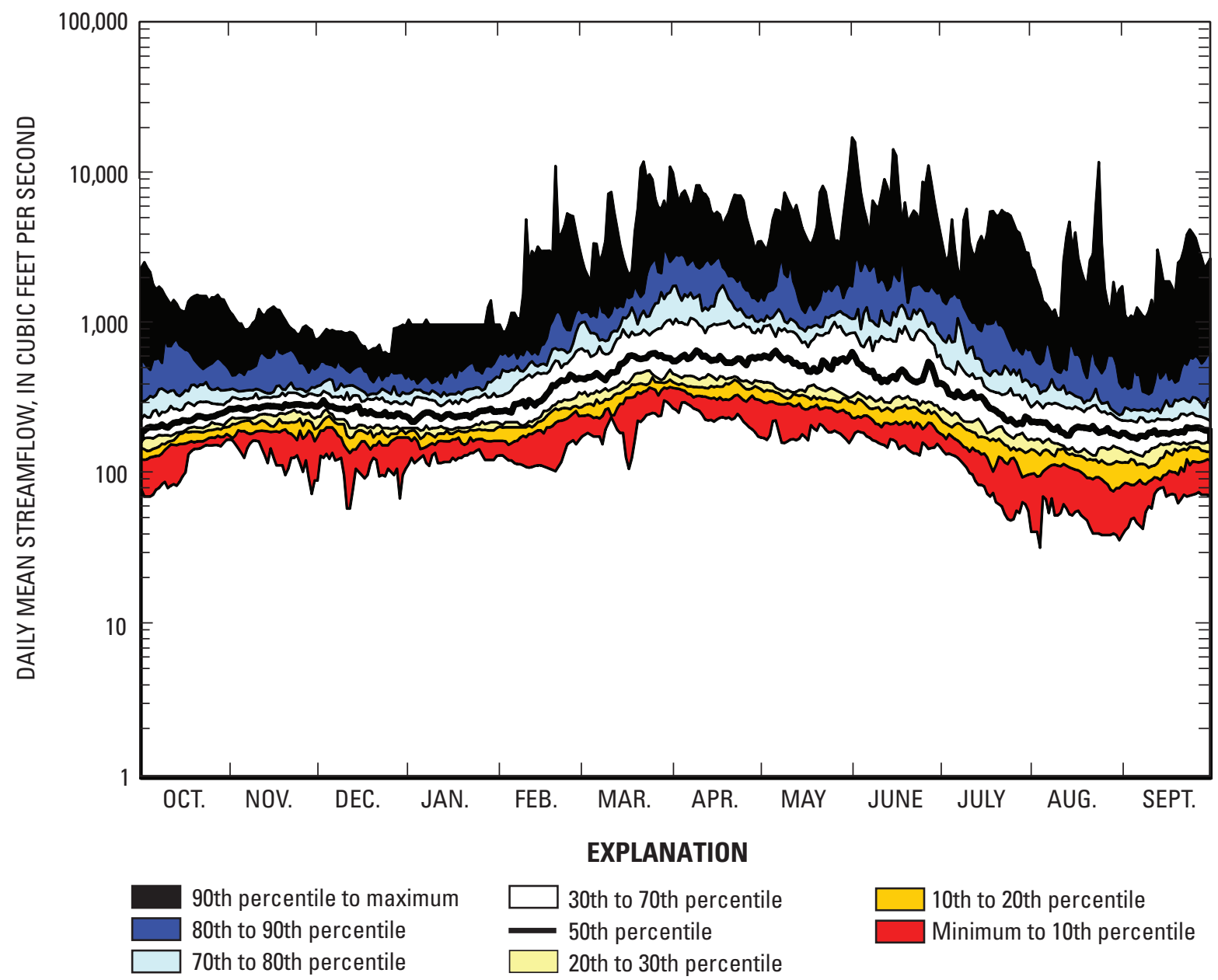


06799000 ELKHORN RIVER AT NORFOLK, NEBR.

Monthly and annual streamflow, 1945-2004

\begin{tabular}{lccccc}
\hline & $\begin{array}{c}\text { Maximum } \\
\left(\mathbf{f t}^{3} / \mathbf{s}\right)\end{array}$ & $\begin{array}{c}\text { Mini- } \\
\mathbf{m u m} \\
\left(\mathbf{f t}^{3} / \mathbf{s}\right)\end{array}$ & $\begin{array}{c}\text { Mean } \\
\left(\mathbf{f t}^{3} / \mathbf{s}\right)\end{array}$ & $\begin{array}{c}\text { Stan- } \\
\text { dard } \\
\text { devia- } \\
\text { tion }\end{array}$ & $\begin{array}{c}\text { Coef- } \\
\text { ficient } \\
\text { of } \\
\text { varia- } \\
\text { tion }\end{array}$ \\
\hline October & 1,418 & 140 & 327 & 280 & 0.86 \\
November & 847 & 169 & 310 & 127 & 0.41 \\
December & 607 & 156 & 273 & 95 & 0.35 \\
January & 1,000 & 158 & 289 & 156 & 0.54 \\
February & 1,862 & 213 & 481 & 327 & 0.68 \\
March & 2,360 & 320 & 813 & 502 & 0.62 \\
April & 3,338 & 341 & 930 & 740 & 0.80 \\
May & 2,682 & 246 & 789 & 566 & 0.72 \\
June & 4,673 & 227 & 1,000 & 1,012 & 1.01 \\
July & 1,479 & 125 & 470 & 358 & 0.76 \\
August & 1,398 & 91 & 330 & 306 & 0.93 \\
September & 1,323 & 87 & 268 & 216 & 0.81 \\
Annual & & & & & \\
\hline
\end{tabular}

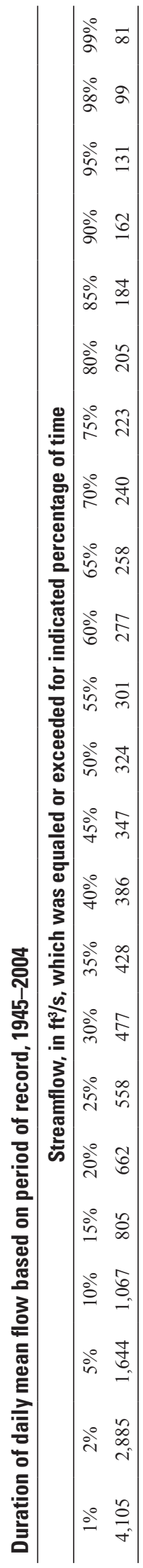

Magnitude and probability of annual low flow based on period of record, 1945-2004

\begin{tabular}{|c|c|c|c|c|c|c|}
\hline \multirow{3}{*}{$\begin{array}{l}\text { Period } \\
\text { (con- } \\
\text { secu- } \\
\text { tive } \\
\text { days) }\end{array}$} & \multicolumn{6}{|c|}{$\begin{array}{l}\text { Streamflow, in } \mathrm{ft}^{3} / \mathrm{s} \text {, for indicated recurrence interval, in } \\
\text { years, and non-exceedance probability, in percent }\end{array}$} \\
\hline & 2 & 5 & 10 & 20 & 50 & 100 \\
\hline & $50 \%$ & $20 \%$ & $10 \%$ & $5 \%$ & $2 \%$ & $1 \%$ \\
\hline 1 & 113 & 76 & 60 & 49 & 38 & 32 \\
\hline 2 & 118 & 79 & 62 & 51 & 40 & 34 \\
\hline 3 & 122 & 81 & 64 & 52 & 41 & 34 \\
\hline 7 & 134 & 88 & 69 & 56 & 44 & 37 \\
\hline 14 & 145 & 95 & 75 & 61 & 48 & 40 \\
\hline 30 & 161 & 109 & 88 & 73 & 58 & 50 \\
\hline 60 & 184 & 129 & 107 & 92 & 78 & 69 \\
\hline 90 & 202 & 144 & 122 & 108 & 94 & 86 \\
\hline 183 & 240 & 182 & 164 & 152 & 143 & 138 \\
\hline \multicolumn{7}{|c|}{ October-November } \\
\hline 1 & 175 & 126 & 107 & 94 & 82 & 75 \\
\hline 2 & 179 & 131 & 113 & 101 & 89 & 83 \\
\hline 3 & 184 & 135 & 116 & 104 & 92 & 86 \\
\hline 7 & 200 & 146 & 126 & 114 & 102 & 95 \\
\hline 14 & 215 & 156 & 136 & 123 & 111 & 104 \\
\hline 30 & 236 & 179 & 160 & 149 & 139 & 135 \\
\hline 60 & 264 & 202 & 184 & 173 & 165 & 161 \\
\hline
\end{tabular}




\section{NORTH FORK ELKHORN RIVER NEAR PIERCE, NEBR.}

LOCATION.--Lat $42^{\circ} 08^{\prime} 54^{\prime \prime}$, long $97^{\circ} 28^{\prime} 43^{\prime \prime}$, in NE 1/1 NW 1/4 sec.18, T.25 N., R.1 W., Pierce County, Hydrologic Unit 10220002, on right bank $4 \mathrm{ft}$ downstream and $25 \mathrm{ft}$ from end of bridge, $4.5 \mathrm{mi}$ southeast of Pierce, and at mile 20.8 .

DRAINAGE AREA.--701 $\mathrm{mi}^{2}$ of which $30 \mathrm{mi}^{2}$ probably is noncontributing.

\section{STREAMFLOW RECORDS}

REVISED RECORDS.--WDR NE-94-1: Drainage area.

PERIOD OF RECORD.--August 1960 to current year.

GAGE.--Water-stage recorder. Datum of gage is 1,542.88 ft above sea level (U.S. Weather Service levels). Aug. 19, 1960 to 0ct. 7, 1997, waterstage recorder at site 2 mi upstream at datum $10.19 \mathrm{ft}$ higher. Data collection platform at station.

REMARKS.--Record good except for estimated daily streamflows, which are poor.

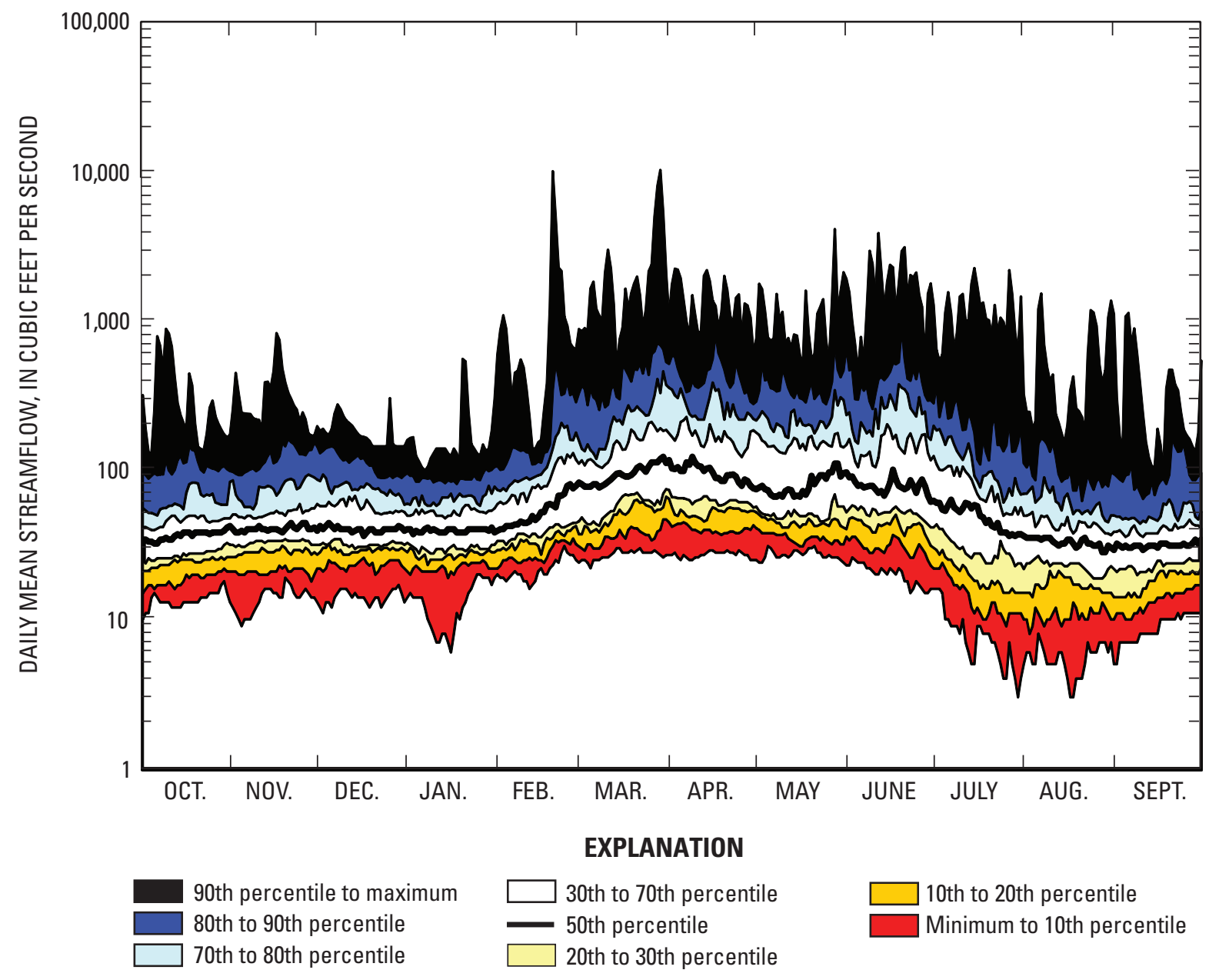


06799100 NORTH FORK ELKHORN RIVER NEAR PIERCE, NEBR.

Monthly and annual streamflow, 1960-2004

\begin{tabular}{lcrrrr}
\hline & $\begin{array}{c}\text { Maximum } \\
\left(\mathbf{f t}^{3} / \mathbf{s}\right)\end{array}$ & $\begin{array}{c}\text { Mini- } \\
\mathbf{m u m} \\
\left(\mathbf{f t}^{3} / \mathbf{s}\right)\end{array}$ & $\begin{array}{c}\text { Mean } \\
\left(\mathbf{f t}^{3} \mathbf{s}\right)\end{array}$ & $\begin{array}{c}\text { Stan- } \\
\text { dard } \\
\text { devia- } \\
\text { tion }\end{array}$ & $\begin{array}{c}\text { Coef- } \\
\text { ficient } \\
\text { of } \\
\text { varia- } \\
\text { tion }\end{array}$ \\
\hline October & 241 & 14 & 52 & 46 & 0.88 \\
November & 233 & 15 & 59 & 50 & 0.85 \\
December & 156 & 15 & 53 & 34 & 0.63 \\
January & 111 & 16 & 49 & 26 & 0.53 \\
February & 834 & 24 & 113 & 149 & 1.32 \\
March & 1,120 & 30 & 200 & 205 & 1.03 \\
April & 1,004 & 29 & 187 & 189 & 1.01 \\
May & 663 & 28 & 162 & 140 & 0.87 \\
June & 799 & 22 & 181 & 174 & 0.96 \\
July & 834 & 12 & 99 & 140 & 1.41 \\
August & 226 & 7 & 54 & 53 & 0.99 \\
September & 191 & 10 & 46 & 43 & 0.94 \\
Annual & $\mathbf{2 8 7}$ & $\mathbf{2 2}$ & $\mathbf{1 0 4}$ & $\mathbf{6 6}$ & $\mathbf{0 . 6 4}$ \\
\hline
\end{tabular}

Magnitude and probability of annual low flow based on period of record, 1960-2004

\begin{tabular}{|c|c|c|c|c|c|c|}
\hline \multirow{3}{*}{$\begin{array}{c}\text { Period } \\
\text { (con- } \\
\text { secu- } \\
\text { tive } \\
\text { days) }\end{array}$} & \multicolumn{6}{|c|}{$\begin{array}{l}\text { Streamflow, in } \mathrm{ft}^{3} / \mathrm{s} \text {, for indicated recurrence interval, } \\
\text { in years, and non-exceedance probability, in percent }\end{array}$} \\
\hline & 2 & 5 & 10 & 20 & 50 & 100 \\
\hline & $50 \%$ & $20 \%$ & $10 \%$ & $5 \%$ & $2 \%$ & $1 \%$ \\
\hline 1 & 18 & 9 & 6 & 4 & 3 & 2 \\
\hline 2 & 19 & 10 & 6 & 4 & 3 & 2 \\
\hline 3 & 20 & 10 & 7 & 5 & 3 & 2 \\
\hline 7 & 21 & 11 & 8 & 6 & 4 & 3 \\
\hline 14 & 23 & 13 & 9 & 7 & 5 & 4 \\
\hline 30 & 26 & 15 & 11 & 9 & 6 & 5 \\
\hline 60 & 29 & 18 & 13 & 10 & 8 & 6 \\
\hline 90 & 32 & 19 & 15 & 12 & 9 & 8 \\
\hline 183 & 39 & 24 & 19 & 16 & 13 & 11 \\
\hline \multicolumn{7}{|c|}{ October-November } \\
\hline 1 & 28 & 19 & 16 & 13 & 11 & 10 \\
\hline 2 & 29 & 19 & 16 & 14 & 12 & 11 \\
\hline 3 & 29 & 20 & 16 & 14 & 12 & 11 \\
\hline 7 & 31 & 20 & 17 & 15 & 13 & 12 \\
\hline 14 & 33 & 22 & 18 & 16 & 14 & 13 \\
\hline 30 & 36 & 23 & 19 & 17 & 15 & 14 \\
\hline 60 & 41 & 25 & 21 & 18 & 15 & 14 \\
\hline
\end{tabular}

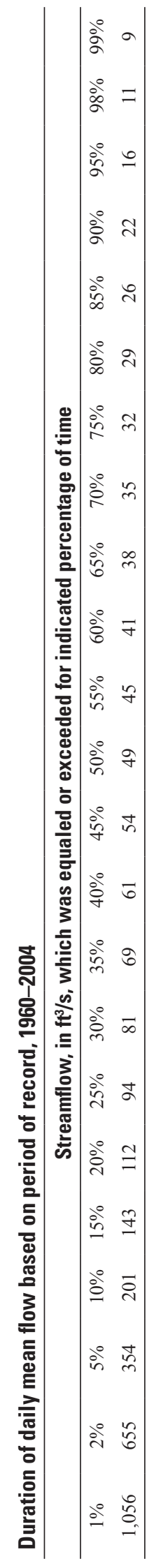


06799350 ELKHORN RIVER AT WEST POINT, NEBR.

LOCATION.--Lat $41^{\circ} 50^{\prime} 22^{\prime \prime}$, long 96 $96^{\circ} 38^{\prime \prime}$, in SW 1/4 NW 1/4 sec.34, T.22 N., R.6 E., Cuming County, Hydrologic Unit 10220003, on right bank near right downstream wing wall of bridge on State Highway 32, 1 mi west of West Point, and at mile 79.8.

DRAINAGE AREA.--4,676 $\mathrm{mi}^{2}$ of which $576 \mathrm{mi}^{2}$ probably is noncontributing.

\section{STREAMFLOW RECORDS}

PERIOD OF RECORD.--October 1972 to current year. March 1960 to September 1972 (no winter records 1960-68) in files of Corps of Engineers. Gage-height records collected since 1940 are in reports of U.S. Weather Service.

GAGE.--Water-stage recorder. Datum of gage is 1,291.26 ft above sea level. Prior to May 18, 1976, at site on left bank $50 \mathrm{ft}$ upstream from bridge at same datum. Data collection platform at station.

REMARKS.--Records good except for estimated daily streamflows, which are poor. Some small diversions above station for irrigation.

EXTREMES FOR PERIOD OF RECORD.--Maximum streamflow estimated 33,000 $\mathrm{ft}^{3}$, June 25, 1969, gage height 13.21 feet; minimum daily, $41 \mathrm{ft}^{3}$, Aug. 31, 1976. Flood of March 31, 1960, reached a stage of 16.09 feet, backwater from ice; observed by Corps of Engineers. Flood stage is 12.00 feet gage datum.

Non-exceedance duration hydrograph for Elkhorn River at West Point, Nebr. (06799350) (1972-2004 period of record)

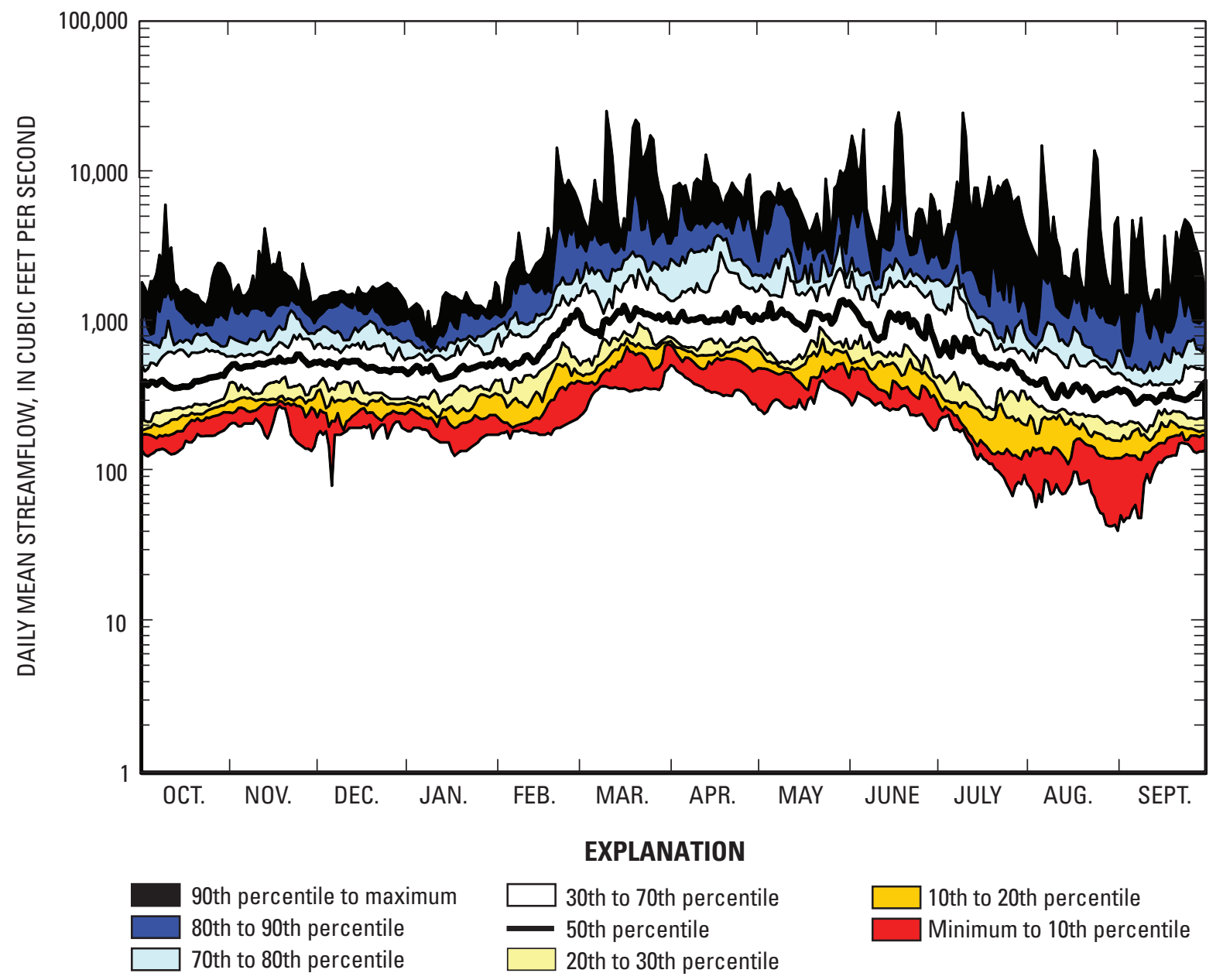


06799350 ELKHORN RIVER AT WEST POINT, NEBR.

Monthly and annual streamflow, 1972-2004

\begin{tabular}{lcrrrr}
\hline & $\begin{array}{c}\text { Maximum } \\
\left(\mathbf{f t}^{3} / \mathbf{s}\right)\end{array}$ & $\begin{array}{c}\text { Mini- } \\
\mathbf{m u m} \\
\left(\mathbf{f t t}^{3} \mathbf{s}\right)\end{array}$ & $\begin{array}{r}\text { Mean } \\
\left(\mathbf{f t}^{3} / \mathbf{s}\right)\end{array}$ & $\begin{array}{c}\text { Stan- } \\
\text { dard } \\
\text { devia- } \\
\text { tion }\end{array}$ & $\begin{array}{c}\text { Coef- } \\
\text { ficient } \\
\text { of } \\
\text { varia- } \\
\text { tion }\end{array}$ \\
\hline October & 1,606 & 174 & 542 & 357 & 0.66 \\
November & 1,802 & 241 & 631 & 353 & 0.56 \\
December & 1,314 & 203 & 588 & 310 & 0.53 \\
January & 1,106 & 168 & 537 & 236 & 0.44 \\
February & 2,744 & 201 & 1,022 & 664 & 0.65 \\
March & 5,256 & 411 & 1,739 & 1,229 & 0.71 \\
April & 6,171 & 378 & 1,786 & 1,428 & 0.80 \\
May & 5,618 & 325 & 1,627 & 1,073 & 0.66 \\
June & 3,844 & 339 & 1,529 & 980 & 0.64 \\
July & 6,945 & 154 & 1,000 & 1,235 & 1.23 \\
August & 1,994 & 90 & 593 & 518 & 0.87 \\
September & 1,646 & 137 & 476 & 354 & 0.74 \\
Annual & $\mathbf{2 , 2 5 3}$ & $\mathbf{3 3 3}$ & $\mathbf{1 , 0 0 0}$ & $\mathbf{5 1 0}$ & $\mathbf{0 . 5 1}$ \\
\hline
\end{tabular}

Magnitude and probability of annual low flow based on period of record, 1972-2004

\begin{tabular}{|c|c|c|c|c|c|c|}
\hline \multirow{3}{*}{$\begin{array}{c}\text { Period } \\
\text { (con- } \\
\text { secu- } \\
\text { tive } \\
\text { days) }\end{array}$} & \multicolumn{6}{|c|}{$\begin{array}{l}\text { Streamflow, in } \mathrm{ft}^{3} / \mathrm{s} \text {, for indicated recurrence interval, } \\
\text { in years, and non-exceedance probability, in percent }\end{array}$} \\
\hline & 2 & 5 & 10 & 20 & 50 & 100 \\
\hline & $50 \%$ & $20 \%$ & $10 \%$ & $5 \%$ & $2 \%$ & $1 \%$ \\
\hline 1 & 188 & 113 & 84 & 64 & 47 & 37 \\
\hline 2 & 193 & 116 & 87 & 67 & 49 & 39 \\
\hline 3 & 199 & 119 & 88 & 67 & 49 & 39 \\
\hline 7 & 218 & 130 & 95 & 73 & 53 & 42 \\
\hline 14 & 240 & 141 & 103 & 78 & 56 & 44 \\
\hline 30 & 272 & 166 & 125 & 98 & 74 & 60 \\
\hline 60 & 329 & 203 & 156 & 124 & 96 & 81 \\
\hline 90 & 374 & 228 & 176 & 142 & 111 & 94 \\
\hline 183 & 457 & 288 & 228 & 188 & 152 & 132 \\
\hline \multicolumn{7}{|c|}{ October-November } \\
\hline 1 & 303 & 201 & 162 & 136 & 112 & 99 \\
\hline 2 & 310 & 206 & 166 & 139 & 115 & 100 \\
\hline 3 & 318 & 210 & 169 & 141 & 115 & 101 \\
\hline 7 & 345 & 221 & 175 & 145 & 117 & 101 \\
\hline 14 & 373 & 234 & 185 & 152 & 123 & 107 \\
\hline 30 & 406 & 260 & 209 & 176 & 147 & 130 \\
\hline 60 & 491 & 313 & 250 & 210 & 173 & 153 \\
\hline
\end{tabular}

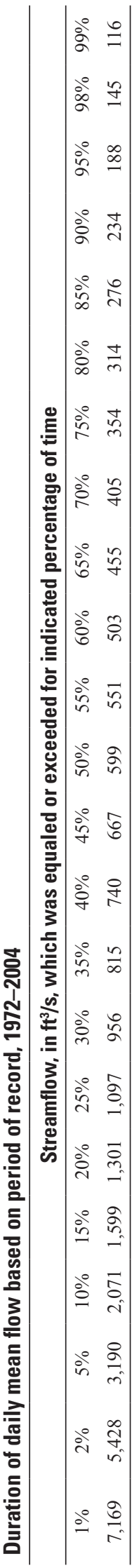


06799385 PEBBLE CREEK AT SCRIBNER, NEBR.

LOCATION.--Lat 4139'32", long 96²41'03", in SW 1/4 NW 1⁄4 sec.34, T.22 N., R.6 E., Dodge County, Hydrologic Unit 10220003.

\section{STREAMFLOW RECORDS}

PERIOD OF RECORD.--October 1993 to current year.

Non-exceedance duration hydrograph for Pebble Creek at Scribner, Nebr. (06799385) (1978-2004 period of record)

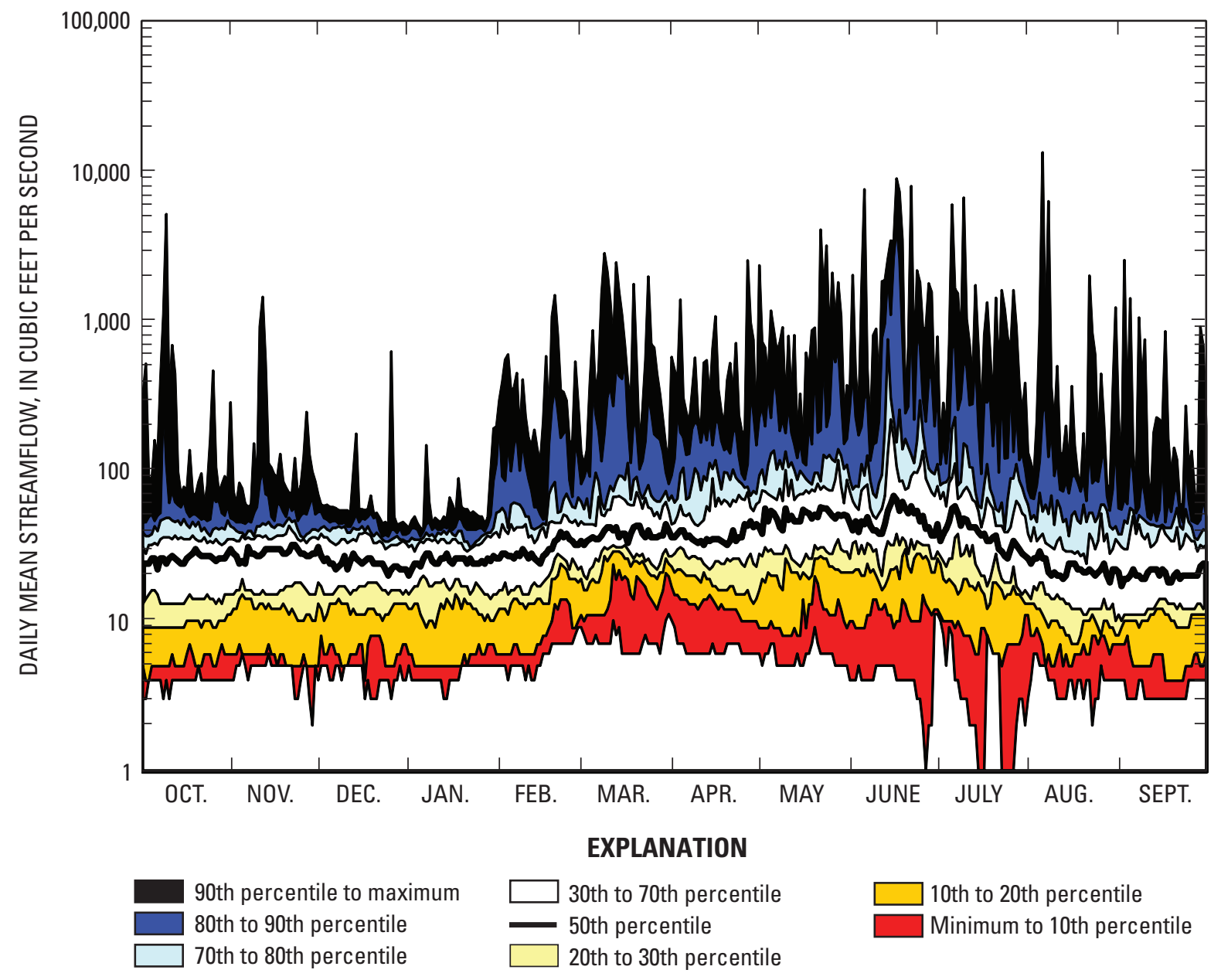


06799385 PEBBLE CREEK AT SCRIBNER, NEBR.

Monthly and annual streamflow, 1978-2004

\begin{tabular}{lrrrrr}
\hline & $\begin{array}{c}\text { Maximum } \\
\left(\mathbf{f t}^{3} / \mathbf{s}\right)\end{array}$ & $\begin{array}{c}\text { Mini- } \\
\mathbf{m u m} \\
\left(\mathbf{f t t}^{3} / \mathbf{s}\right)\end{array}$ & $\begin{array}{c}\text { Mean } \\
\left(\mathbf{f t}^{3} / \mathbf{s}\right)\end{array}$ & $\begin{array}{c}\text { Stan- } \\
\text { dard } \\
\text { devia- } \\
\text { tion }\end{array}$ & $\begin{array}{c}\text { Coef- } \\
\text { ficient } \\
\text { of } \\
\text { varia- } \\
\text { tion }\end{array}$ \\
\hline October & 55 & 12 & 34 & 14 & 0.42 \\
November & 108 & 13 & 41 & 25 & 0.62 \\
December & 51 & 13 & 33 & 12 & 0.35 \\
January & 48 & 13 & 34 & 11 & 0.33 \\
February & 103 & 27 & 52 & 22 & 0.43 \\
March & 130 & 26 & 62 & 30 & 0.48 \\
April & 206 & 32 & 74 & 53 & 0.72 \\
May & 257 & 28 & 123 & 75 & 0.61 \\
June & 496 & 27 & 187 & 160 & 0.86 \\
July & 399 & 22 & 110 & 108 & 0.98 \\
August & 549 & 14 & 113 & 172 & 1.52 \\
September & 131 & 12 & 40 & 34 & 0.84 \\
Annual & $\mathbf{1 4 4}$ & $\mathbf{3 8}$ & $\mathbf{7 5}$ & $\mathbf{3 9}$ & $\mathbf{0 . 5 2}$ \\
\hline
\end{tabular}

Magnitude and probability of annual low flow based on period of record, 1978-2004

\begin{tabular}{|c|c|c|c|c|c|c|}
\hline \multirow{3}{*}{$\begin{array}{c}\text { Period } \\
\text { (con- } \\
\text { secu- } \\
\text { tive } \\
\text { days) }\end{array}$} & \multicolumn{6}{|c|}{$\begin{array}{l}\text { Streamflow, in } \mathrm{ft}^{3} / \mathrm{s} \text {, for indicated recurrence interval, } \\
\text { in years, and non-exceedance probability, in percent }\end{array}$} \\
\hline & 2 & 5 & 10 & 20 & 50 & 100 \\
\hline & $50 \%$ & $20 \%$ & $10 \%$ & $5 \%$ & $2 \%$ & $1 \%$ \\
\hline 1 & 13 & 5 & 2 & 1 & 0 & 0 \\
\hline 2 & 13 & 5 & 2 & 1 & 1 & 0 \\
\hline 3 & 14 & 5 & 3 & 1 & 1 & 0 \\
\hline 7 & 14 & 6 & 3 & 2 & 1 & 0 \\
\hline 14 & 15 & 7 & 4 & 2 & 1 & 1 \\
\hline 30 & 16 & 8 & 5 & 4 & 2 & 2 \\
\hline 60 & 20 & 11 & 7 & 5 & 3 & 2 \\
\hline 90 & 22 & 12 & 8 & 6 & 4 & 3 \\
\hline 183 & 28 & 14 & 10 & 7 & 4 & 3 \\
\hline \multicolumn{7}{|c|}{ October-November } \\
\hline 1 & 17 & 8 & 5 & 3 & 2 & 1 \\
\hline 2 & 17 & 8 & 5 & 3 & 2 & 1 \\
\hline 3 & 17 & 8 & 5 & 4 & 2 & 2 \\
\hline 7 & 18 & 9 & 6 & 4 & 3 & 2 \\
\hline 14 & 20 & 10 & 6 & 4 & 3 & 2 \\
\hline 30 & 22 & 11 & 7 & 5 & 3 & 2 \\
\hline 60 & 26 & 12 & 8 & 6 & 4 & 3 \\
\hline
\end{tabular}

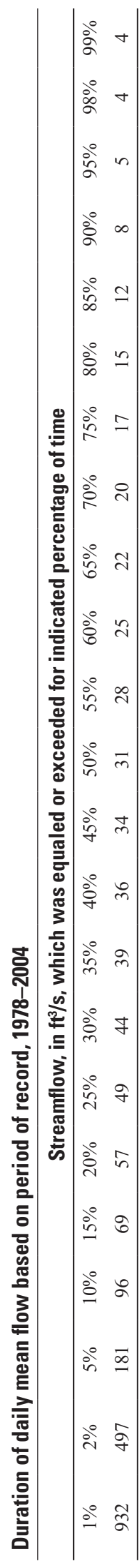


06799500 LOGAN CREEK NEAR UEHLING, NEBR.

LOCATION.--Lat 41 ${ }^{\circ} 42^{\prime} 46^{\prime \prime}$, long 96 $31^{\prime} 19^{\prime \prime}$, in SE 1/4 SE 1/4 sec.9, T.20 N., R.8 E., Dodge County, Hydrologic Unit 10220004, near left bank on upstream side of bridge on county road, 2 mi southwest of Uehling and 8.8 mi upstream from mouth.

DRAINAGE AREA.--1015 mi².

REVISED RECORDS.--WDR NE-94-1: Drainage Area.

\section{STREAMFLOW RECORDS}

PERIOD OF RECORD.--March 1941 to current year.

GAGE.--Water-stage recorder. Datum of gage is 1,208.73 ft above sea level. See WSP 1918 for history of changes prior to July 15, 1963. July 16, 1963 to Mar. 27. 1989, near right bank on downstream side of bridge at present site and datum. Mar. 28, 1989 to Mar. 22, 1990, 250 ft upstream on left bank at same datum. Data collection platform at station.

COOPERATION.--Records provided by Nebraska Department of Natural Resources and reviewed by the U.S. Geological Survey.

REMARKS.--Records good except for estimated daily streamflows, which are poor.

Non-exceedance duration hydrograph for Logan Creek near Uehling, Nebr. (06799500) (1941-2004 period of record)

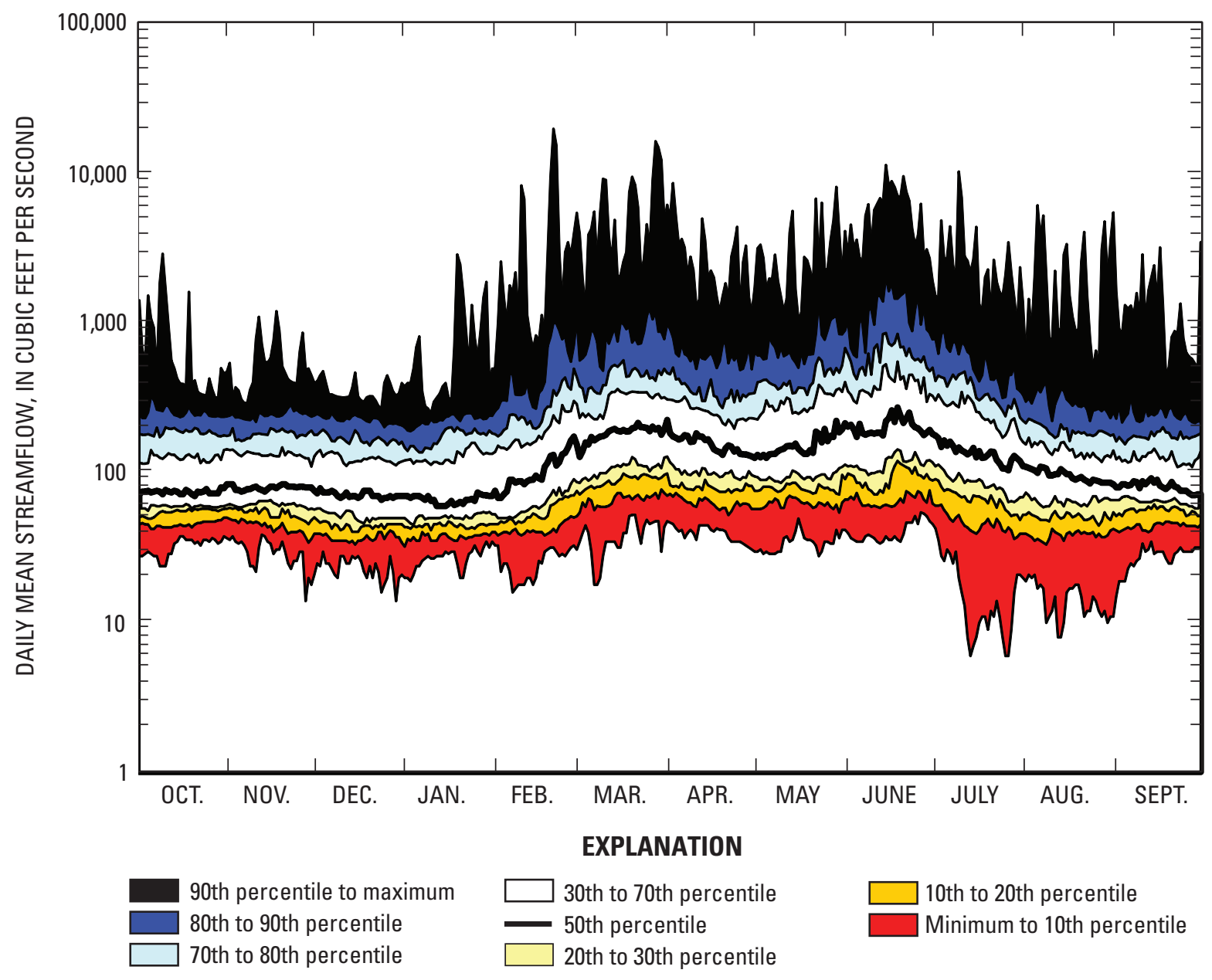


06799500 LOGAN CREEK NEAR UEHLING, NEBR.

Monthly and annual streamflow, 1941-2004

\begin{tabular}{|c|c|c|c|c|c|c|}
\hline & \multicolumn{2}{|c|}{$\begin{array}{l}\text { Maximum } \\
\quad\left(\mathrm{ft}^{3} / \mathrm{s}\right)\end{array}$} & $\begin{array}{l}\text { Mini- } \\
\text { mum } \\
\left(\mathrm{ft}^{3} / \mathrm{s}\right)\end{array}$ & $\begin{array}{l}\text { Mean } \\
\left(\mathrm{ft}^{3} / \mathrm{s}\right)\end{array}$ & $\begin{array}{c}\text { Stan- } \\
\text { dard } \\
\text { devia- } \\
\text { tion }\end{array}$ & $\begin{array}{l}\text { Coef- } \\
\text { ficient } \\
\text { of } \\
\text { varia- } \\
\text { tion }\end{array}$ \\
\hline October & \multicolumn{2}{|c|}{499} & 33 & 123 & 99 & 0.80 \\
\hline November & \multicolumn{2}{|c|}{453} & 38 & 118 & 87 & 0.74 \\
\hline December & \multicolumn{2}{|c|}{337} & 32 & 103 & 73 & 0.70 \\
\hline January & \multicolumn{2}{|c|}{583} & 34 & 111 & 95 & 0.85 \\
\hline February & \multicolumn{2}{|c|}{2,177} & 38 & 263 & 320 & 1.22 \\
\hline March & \multicolumn{2}{|c|}{2,388} & 57 & 409 & 408 & 1.00 \\
\hline April & \multicolumn{2}{|c|}{1,742} & 43 & 287 & 318 & 1.11 \\
\hline May & \multicolumn{2}{|c|}{1,417} & 40 & 324 & 272 & 0.84 \\
\hline June & \multicolumn{2}{|c|}{2,766} & 57 & 502 & 492 & 0.98 \\
\hline July & \multicolumn{2}{|c|}{1,843} & 17 & 266 & 271 & 1.02 \\
\hline August & \multicolumn{2}{|c|}{1,056} & 15 & 165 & 179 & 1.08 \\
\hline September & \multicolumn{2}{|c|}{613} & 32 & 133 & 115 & 0.87 \\
\hline Annual & \multicolumn{2}{|c|}{710} & 66 & 234 & 141 & 0.60 \\
\hline \multicolumn{7}{|c|}{$\begin{array}{l}\text { Magnitude and probability of annual low flow based on period } \\
\text { of record, 1941-2004 }\end{array}$} \\
\hline \multirow{3}{*}{$\begin{array}{l}\text { Period } \\
\text { (con- } \\
\text { secu- } \\
\text { tive } \\
\text { days) }\end{array}$} & \multicolumn{6}{|c|}{$\begin{array}{l}\text { Streamflow, in } \mathrm{ft}^{3} / \mathrm{s} \text {, for indicated recurrence interval, } \\
\text { in years, and non-exceedance probability, in percent }\end{array}$} \\
\hline & 2 & 5 & 10 & 20 & 50 & 100 \\
\hline & $50 \%$ & $20 \%$ & $10 \%$ & $5 \%$ & $2 \%$ & $1 \%$ \\
\hline 1 & 44 & 24 & 17 & 13 & 10 & 8 \\
\hline 2 & 45 & 25 & 18 & 14 & 11 & 9 \\
\hline 3 & 46 & 26 & 19 & 15 & 11 & 9 \\
\hline 7 & 50 & 29 & 22 & 17 & 13 & 12 \\
\hline 14 & 55 & 31 & 24 & 19 & 15 & 13 \\
\hline 30 & 61 & 37 & 29 & 24 & 20 & 18 \\
\hline 60 & 69 & 42 & 33 & 27 & 22 & 20 \\
\hline 90 & 74 & 46 & 37 & 31 & 26 & 23 \\
\hline 183 & 88 & 54 & 43 & 36 & 30 & 27 \\
\hline \multicolumn{7}{|c|}{ October-November } \\
\hline 1 & 62 & 35 & 26 & 21 & 16 & 14 \\
\hline 2 & 64 & 37 & 28 & 22 & 17 & 15 \\
\hline 3 & 66 & 38 & 29 & 24 & 19 & 17 \\
\hline 7 & 70 & 42 & 34 & 28 & 24 & 21 \\
\hline 14 & 74 & 46 & 37 & 32 & 27 & 24 \\
\hline 30 & 80 & 51 & 42 & 36 & 31 & 28 \\
\hline 60 & 90 & 56 & 45 & 38 & 33 & 30 \\
\hline
\end{tabular}

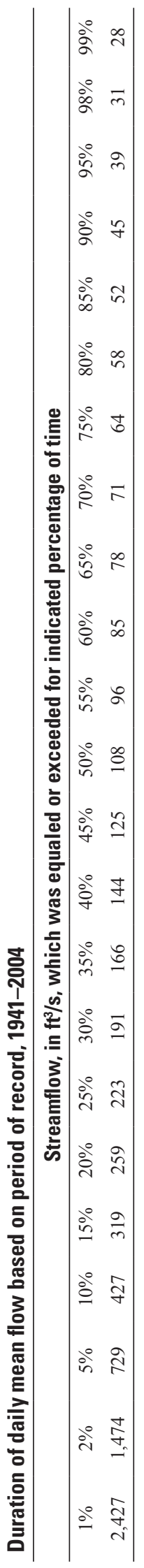




\section{MAPLE CREEK NEAR NICKERSON, NEBR.}

LOCATION.--Lat 41 ${ }^{\circ} 33^{\prime} 37^{\prime \prime}$, long 96³2'27", in SW 1/4 NW 1/4 sec.4, T.18 N., R.8 E., Dodge County, Hydrologic Unit 10220003, on right bank 8 ft downstream from county road bridge 2 mi upstream from U.S. Highways 77 and 275, 5 mi northwest of Nickerson, and 4 mi upstream from mouth.

DRAINAGE AREA.--368 $\mathrm{mi}^{2}$.

\section{STREAMFLOW RECORDS}

REVISED RECORDS.--WSP 1630: 1957-58. WDR NE-98: Drainage area.

PERIOD OF RECORD.--October 1951 to current year.

GAGE.--Water-stage recorder. Datum of gage is $1,211.62 \mathrm{ft}$ above sea level. Prior to July 28, 1960, non-recording gage at highway bridge, July 28, 1960 to July 28, 1987, water-stage recorder $180 \mathrm{ft}$ upstream from highway bridge and July 29, 1987 to July 23, 1991 water-stage recorder $30 \mathrm{ft}$ downstream from highway bridge. All at/near U.S. Highway 77 bridge, 2 mi downstream from present gage, at datum $17.06 \mathrm{ft}$ lower. Data collection platform at station.

REMARKS.--Records good except for estimated daily streamflows, which are poor.

EXTREMES FOR PERIOD OF RECORD.--Maximum streamflow, 13,700 ft ${ }^{3}$, Aug. 6, 1996, (gage height 17.33 feet, (gage datum); maximum gage height, 17.65 feet, (gage datum), June 17, 1984 from flood mark, minimum daily streamflow, $0.1 \mathrm{ft}^{3}$, Jan. 15, 16, 1956.

Non-exceedance duration hydrograph for Maple Creek near Nickerson, Nebr. (06800000) (1951-2004 period of record)

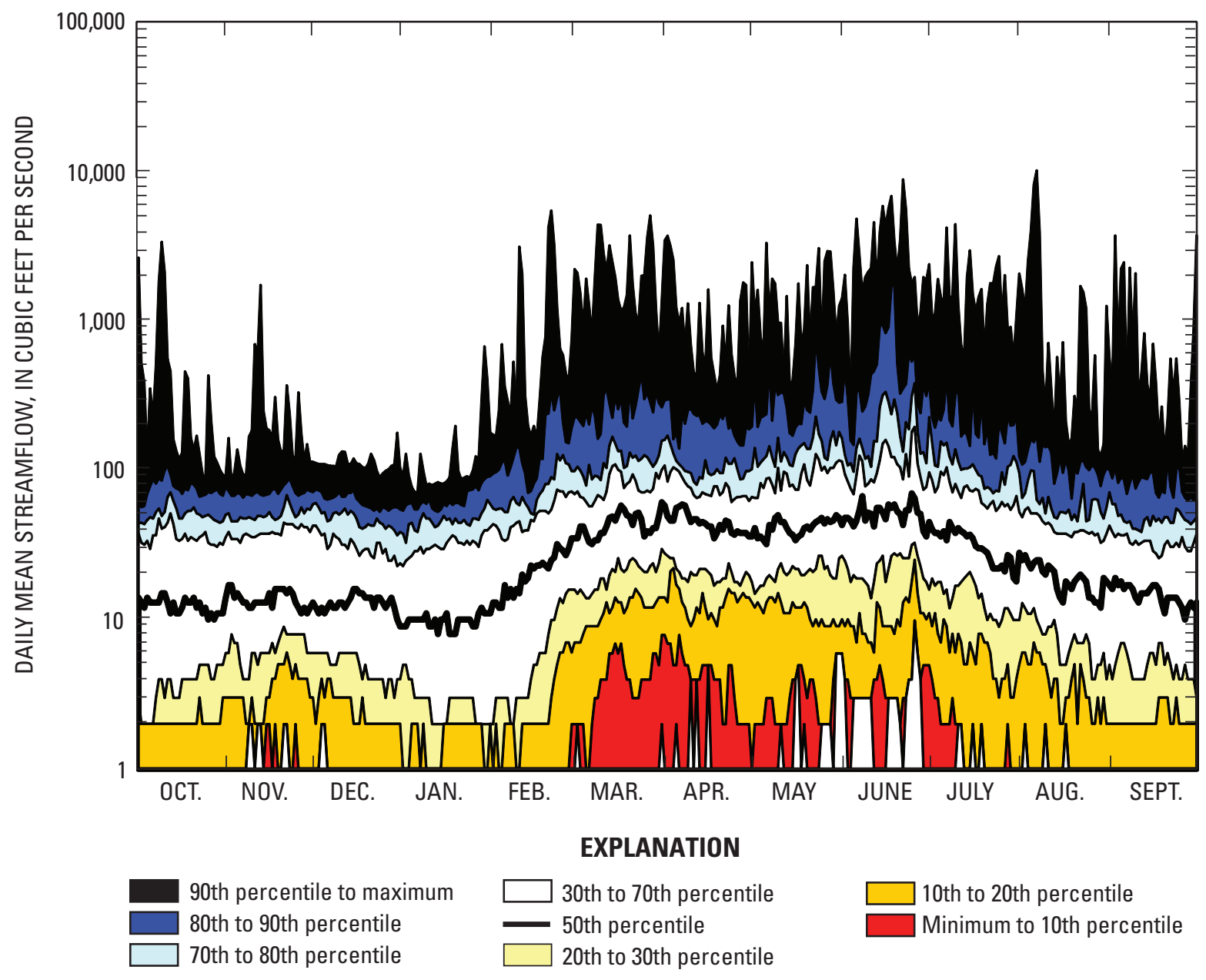


06800000 MAPLE CREEK NEAR NICKERSON, NEBR.

Monthly and annual streamflow, 1951-2005

\begin{tabular}{lccrcc}
\hline & $\begin{array}{c}\text { Maximum } \\
\left(\mathbf{f t}^{3} / \mathbf{s}\right)\end{array}$ & $\begin{array}{c}\text { Mini- } \\
\mathbf{m u m} \\
\left(\mathbf{f t t}^{3} / \mathbf{s}\right)\end{array}$ & $\begin{array}{c}\text { Mean } \\
\left(\mathbf{f t}^{3} / \mathbf{s}\right)\end{array}$ & $\begin{array}{c}\text { Stan- } \\
\text { dard } \\
\text { devia- } \\
\text { tion }\end{array}$ & $\begin{array}{c}\text { Coef- } \\
\text { ficient } \\
\text { of } \\
\text { varia- } \\
\text { tion }\end{array}$ \\
\hline October & 323 & 0 & 37 & 55 & 1.50 \\
November & 158 & 1 & 30 & 35 & 1.16 \\
December & 102 & 1 & 24 & 25 & 1.07 \\
January & 83 & 0 & 24 & 25 & 1.06 \\
February & 446 & 1 & 69 & 82 & 1.19 \\
March & 674 & 1 & 131 & 153 & 1.17 \\
April & 590 & 1 & 94 & 122 & 1.30 \\
May & 642 & 1 & 124 & 134 & 1.08 \\
June & 1,252 & 3 & 212 & 273 & 1.28 \\
July & 1,023 & 1 & 102 & 159 & 1.56 \\
August & 762 & 1 & 67 & 124 & 1.85 \\
September & 383 & 0 & 46 & 72 & 1.57 \\
Annual & $\mathbf{2 6 4}$ & $\mathbf{5}$ & $\mathbf{7 9}$ & $\mathbf{6 0}$ & $\mathbf{0 . 7 6}$ \\
\hline
\end{tabular}

Magnitude and probability of annual low flow based on period of record, 1951-2004

\begin{tabular}{|c|c|c|c|c|c|c|}
\hline \multirow{3}{*}{$\begin{array}{c}\text { Period } \\
\text { (con- } \\
\text { secu- } \\
\text { tive } \\
\text { days) }\end{array}$} & \multicolumn{6}{|c|}{$\begin{array}{l}\text { Streamflow, in } \mathrm{ft}^{3} / \mathrm{s} \text {, for indicated recurrence interval, } \\
\text { in years, and non-exceedance probability, in percent }\end{array}$} \\
\hline & 2 & 5 & 10 & 20 & 50 & 100 \\
\hline & $\mathbf{5 0} \%$ & $20 \%$ & $10 \%$ & $5 \%$ & $2 \%$ & $1 \%$ \\
\hline 1 & 3 & 1 & 0 & 0 & 0 & 0 \\
\hline 2 & 3 & 1 & 0 & 0 & 0 & 0 \\
\hline 3 & 3 & 1 & 0 & 0 & 0 & 0 \\
\hline 7 & 3 & 1 & 0 & 0 & 0 & 0 \\
\hline 14 & 4 & 1 & 0 & 0 & 0 & 0 \\
\hline 30 & 6 & 1 & 1 & 0 & 0 & 0 \\
\hline 60 & 8 & 2 & 1 & 1 & 0 & 0 \\
\hline 90 & 10 & 3 & 1 & 1 & 0 & 0 \\
\hline 183 & 15 & 4 & 2 & 1 & 0 & 0 \\
\hline \multicolumn{7}{|c|}{ October-November } \\
\hline 1 & 6 & 1 & 1 & 0 & 0 & 0 \\
\hline 2 & 6 & 2 & 1 & 0 & 0 & 0 \\
\hline 3 & 7 & 2 & 1 & 0 & 0 & 0 \\
\hline 7 & 8 & 2 & 1 & 0 & 0 & 0 \\
\hline 14 & 9 & 2 & 1 & 1 & 0 & 0 \\
\hline 30 & 11 & 3 & 1 & 1 & 0 & 0 \\
\hline 60 & 15 & 4 & 2 & 1 & 1 & 0 \\
\hline
\end{tabular}

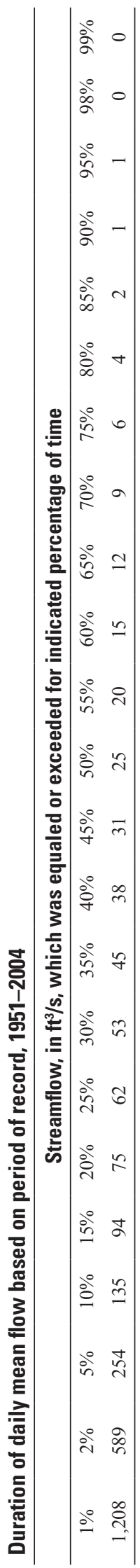




\section{ELKHORN RIVER AT WATERLO0, NEBR.}

LOCATION.--Lat $41^{\circ} 17^{\prime} 36^{\prime \prime}$, long 96¹7'02", in SE 1/4 SW 1/4 sec.3, T.15 N., R.10 E., Douglas County, Hydrologic Unit 10220003, on right bank at Nebraska Highway 64 bridge at north edge of Waterloo, 3.5 mi downstream from Rawhide Creek, and at mile 13.8.

DRAINAGE AREA.--6,900 $\mathrm{mi}^{2}$ of which $1,030 \mathrm{mi}^{2}$ probably is noncontributing.

\section{STREAMFLOW RECORDS}

REVISED RECORDS.--WSP 1390: 1914 (M), 1915, 1936, 1943(M). WDR NE-94-1: Drainage area.

PERIOD OF RECORD.--April 1899 to November 1903, May 1911 to September 1915, August 1928 to current year. Published as "at Arlington" 18991903, July 1913 to September 1915. Monthly streamflow only for some periods, published in WSP 1310.

GAGE.--Water-stage recorder. Datum of gage is 1,104.73 ft above sea level. Oct. 1, 1960, to July 27, 1978, at datum $2.00 \mathrm{ft} \mathrm{higher.} \mathrm{See} \mathrm{WSP} 1918$ for history of changes prior to Oct. 1, 1960. July 28, 1978 to Nov. 17, 1993, at site $800 \mathrm{ft}$ downstream at present datum. Data collection platform at station.

REMARKS.--Records good except for estimated daily streamflows, which are poor. Some small diversions above station for irrigation.

EXTREMES FOR PERIOD OF RECORD.--1899 to 1903, 1911-15, 1928-65: Maximum streamflow, 100,000 ft June 12, 1944 (gage height, 16.6 feet, from flood mark in the gage well, site and datum then in use), from rating curve extended from above $22,000 \mathrm{ft}^{3}$, on the basis of current meter measurements of the peak flow in the main channel and velocity/area studies of the overflow section; minimum observed, $50 \mathrm{ft}^{3}$ Nov. $12,1940$. Stage and streamflow of the flood of June 12, 1944, are the greatest known since at least 1880.

Non-exceedance duration hydrograph for Elkhorn River at Waterloo, Nebr. (06800500) (1928-2004 period of record)

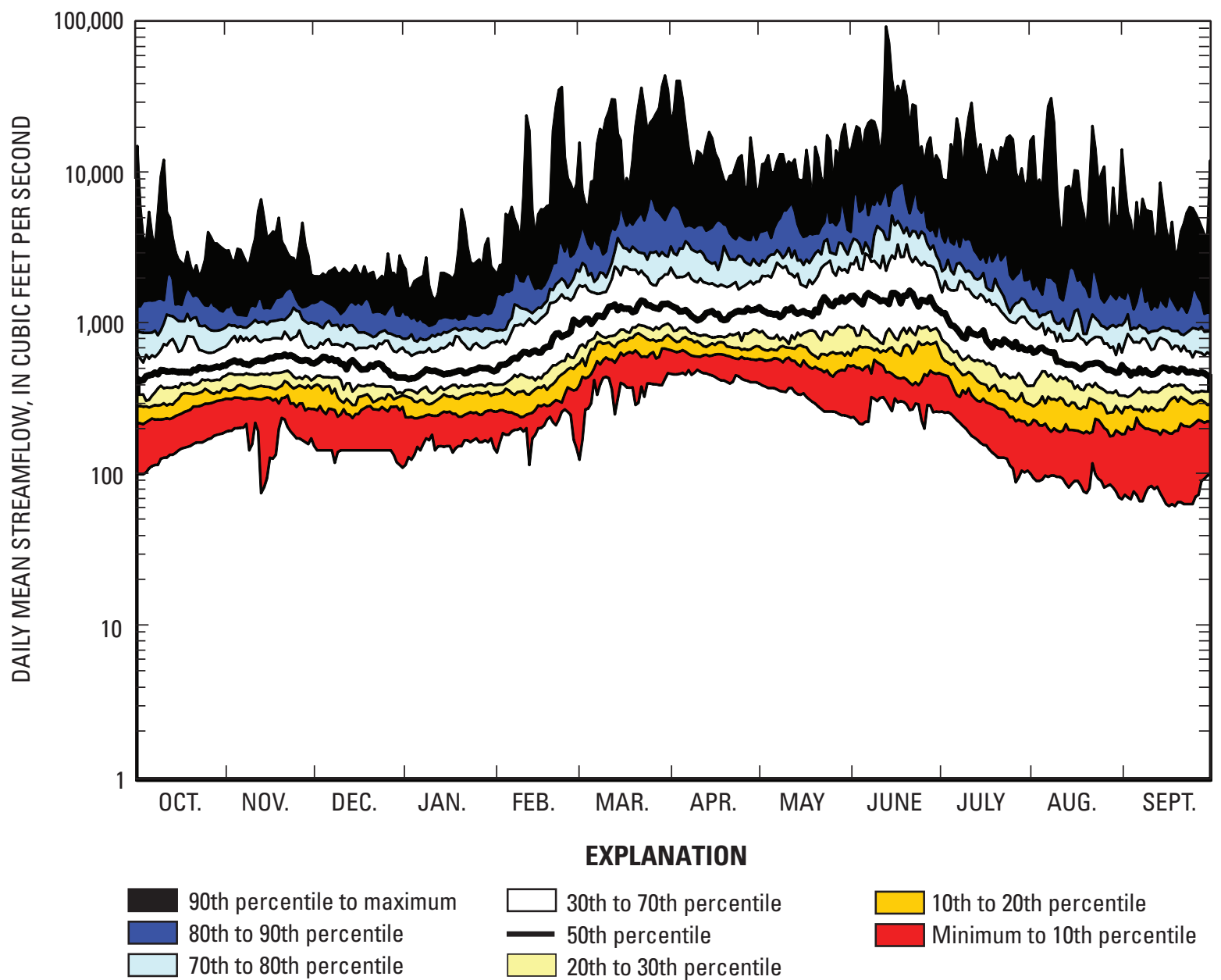


Monthly and annual streamflow, 1928-2005

\begin{tabular}{|c|c|c|c|c|c|c|}
\hline & \multicolumn{2}{|c|}{$\begin{array}{l}\text { Maximum } \\
\quad\left(\mathrm{ft}^{3} / \mathrm{s}\right)\end{array}$} & $\begin{array}{l}\text { Mini- } \\
\text { mum } \\
\left(\mathrm{ft}^{3} / \mathrm{s}\right)\end{array}$ & $\begin{array}{l}\text { Mean } \\
\left(\mathrm{ft}^{3} / \mathrm{s}\right)\end{array}$ & $\begin{array}{c}\text { Stan- } \\
\text { dard } \\
\text { devia- } \\
\text { tion }\end{array}$ & $\begin{array}{l}\text { Coef- } \\
\text { ficient } \\
\text { of } \\
\text { varia- } \\
\text { tion }\end{array}$ \\
\hline October & \multicolumn{2}{|c|}{2,780} & 150 & 725 & 566 & 0.78 \\
\hline November & \multicolumn{2}{|c|}{2,792} & 240 & 761 & 482 & 0.63 \\
\hline December & \multicolumn{2}{|c|}{1,803} & 150 & 669 & 388 & 0.58 \\
\hline January & \multicolumn{2}{|c|}{1,650} & 180 & 631 & 353 & 0.56 \\
\hline February & \multicolumn{2}{|c|}{6,439} & 257 & 1,205 & 1,018 & 0.84 \\
\hline March & \multicolumn{2}{|c|}{8,082} & 489 & 2,237 & 1,774 & 0.79 \\
\hline April & \multicolumn{2}{|c|}{10,450} & 512 & 2,060 & 1,878 & 0.91 \\
\hline May & \multicolumn{2}{|c|}{7,565} & 327 & 2,100 & 1,530 & 0.73 \\
\hline June & \multicolumn{2}{|c|}{11,950} & 405 & 2,830 & 2,484 & 0.88 \\
\hline July & \multicolumn{2}{|c|}{11,470} & 173 & 1,444 & 1,506 & 1.04 \\
\hline August & \multicolumn{2}{|c|}{4,755} & 117 & 943 & 943 & 1.00 \\
\hline September & \multicolumn{2}{|c|}{2,705} & 88 & 725 & 557 & 0.77 \\
\hline Annual & \multicolumn{2}{|c|}{3,870} & 417 & 1,359 & 774 & 0.57 \\
\hline \multicolumn{7}{|c|}{$\begin{array}{l}\text { Magnitude and probability of annual low flow based on period } \\
\text { of record, 1928-2004 }\end{array}$} \\
\hline \multirow{3}{*}{$\begin{array}{l}\text { Period } \\
\text { (con- } \\
\text { secu- } \\
\text { tive } \\
\text { days) }\end{array}$} & \multicolumn{6}{|c|}{$\begin{array}{l}\text { Streamflow, in } \mathrm{ft}^{3} / \mathrm{s} \text {, for indicated recurrence interval, } \\
\text { in years, and non-exceedance probability, in percent }\end{array}$} \\
\hline & 2 & 5 & 10 & 20 & 50 & 100 \\
\hline & $50 \%$ & $20 \%$ & $10 \%$ & $5 \%$ & $2 \%$ & $1 \%$ \\
\hline 1 & 245 & 147 & 114 & 93 & 74 & 64 \\
\hline 2 & 256 & 153 & 118 & 96 & 76 & 65 \\
\hline 3 & 263 & 158 & 121 & 98 & 78 & 66 \\
\hline 7 & 293 & 174 & 133 & 106 & 82 & 69 \\
\hline 14 & 329 & 194 & 146 & 115 & 87 & 72 \\
\hline 30 & 373 & 223 & 169 & 134 & 102 & 85 \\
\hline 60 & 431 & 268 & 210 & 171 & 137 & 118 \\
\hline 90 & 477 & 306 & 246 & 207 & 172 & 152 \\
\hline \multirow[t]{2}{*}{183} & 562 & 365 & 298 & 255 & 216 & 195 \\
\hline & \multicolumn{5}{|c|}{ October-November } & \\
\hline 1 & 368 & 223 & 172 & 138 & 109 & 93 \\
\hline 2 & 382 & 233 & 181 & 146 & 115 & 98 \\
\hline 3 & 392 & 241 & 187 & 151 & 120 & 102 \\
\hline 7 & 432 & 269 & 212 & 174 & 140 & 121 \\
\hline 14 & 472 & 297 & 236 & 197 & 162 & 142 \\
\hline 30 & 520 & 333 & 269 & 228 & 191 & 171 \\
\hline 60 & 585 & 375 & 306 & 262 & 223 & 202 \\
\hline
\end{tabular}

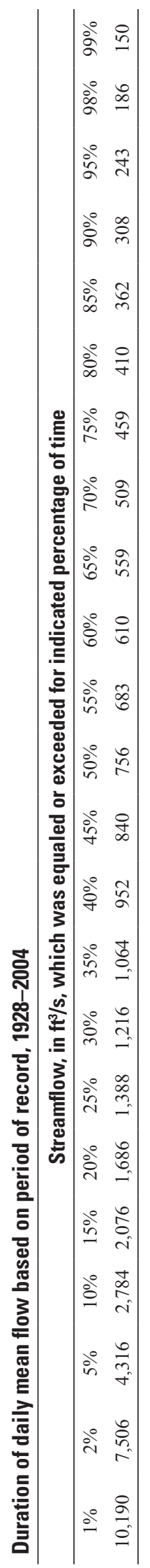




\section{SALT CREEK AT GREENWOOD, NEBR.}

LOCATION.--Lat 40 57'56", long 96²7'16", in SW 1/4 NE 1/4 sec.31, T.12 N., R.9 E., Cass County, Hydrologic Unit 10200203, on right bank just downstream from county road bridge, 0.5 mi west of Greenwood, and at mile 13.0.

DRAINAGE AREA.--1050 mi².

\section{STREAMFLOW RECORDS}

REVISED RECORDS.--WDR NE-94-1: Drainage area.

PERIOD OF RECORD.--November 1951 to current year. Records furnished by Corps of Engineers prior to 0ct. 1, 1972.

GAGE.--Water-stage recorder. Datum of gage is 1,066.14 ft above sea level. Datum lowered 2.00 ft Feb. 6, 2002. Prior to Nov. 5, 1964, non-recording gage at same site. Data collection platform at station.

REMARKS.--Records good except for estimated daily streamflows, which are poor.

EXTREMES FOR PERIOD OF RECORD.--Maximum streamflow 41,000 ft ${ }^{3}$, June 24, 1963, G.H. 23.46 feet; maximum gage height, 23.50 feet, 0 ct. 11, 1973, from flood mark; minimum daily streamflow $14 \mathrm{ft}^{3}$, Jan. 10, 1957.

Non-exceedance duration hydrograph for Salt Creek at Greenwood, Nebr. (06803555) (1951-2004 period of record)

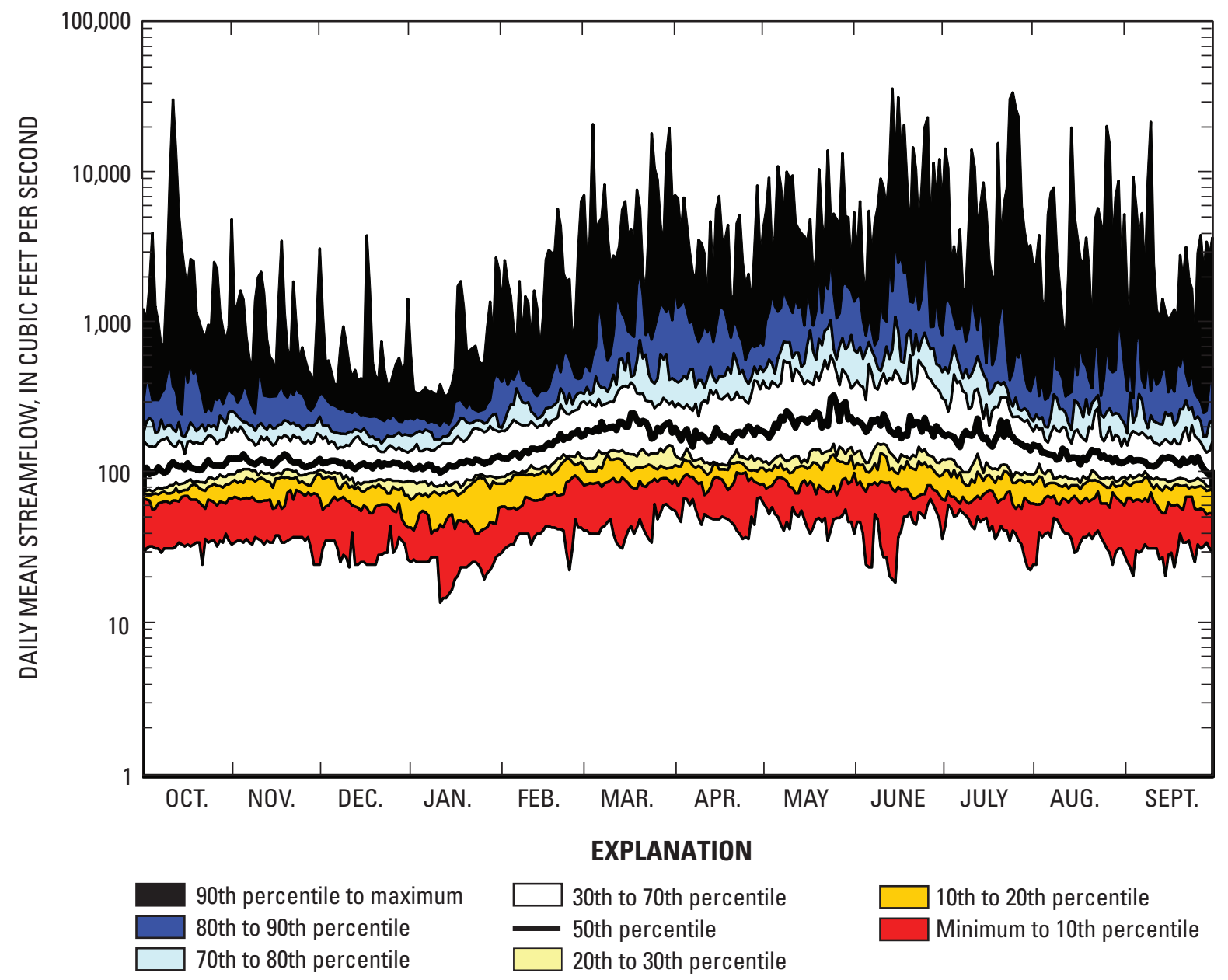


06803555 SALT CREEK AT GREENWOOD, NEBR.

Monthly and annual streamflow, 1951-2004

\begin{tabular}{lrrrrr}
\hline & $\begin{array}{c}\text { Maximum } \\
\left(\mathbf{f t}^{3} / \mathbf{s}\right)\end{array}$ & $\begin{array}{c}\text { Mini- } \\
\mathbf{m u m} \\
\left(\mathbf{f t t}^{3} / \mathbf{s}\right)\end{array}$ & $\begin{array}{c}\text { Mean } \\
\left(\mathbf{f t}^{3} / \mathbf{s}\right)\end{array}$ & $\begin{array}{c}\text { Stan- } \\
\text { dard } \\
\text { devia- } \\
\text { tion }\end{array}$ & $\begin{array}{c}\text { Coef- } \\
\text { ficient } \\
\text { of } \\
\text { varia- } \\
\text { tion }\end{array}$ \\
\hline October & 2,681 & 36 & 247 & 450 & 1.82 \\
November & 638 & 35 & 176 & 116 & 0.66 \\
December & 465 & 37 & 146 & 89 & 0.61 \\
January & 520 & 26 & 157 & 105 & 0.67 \\
February & 952 & 41 & 261 & 182 & 0.70 \\
March & 3,481 & 51 & 489 & 602 & 1.23 \\
April & 2,023 & 58 & 397 & 421 & 1.06 \\
May & 2,383 & 55 & 591 & 591 & 1.00 \\
June & 4,101 & 66 & 698 & 768 & 1.10 \\
July & 5,461 & 56 & 501 & 781 & 1.56 \\
August & 1,748 & 43 & 307 & 340 & 1.11 \\
September & 1,534 & 53 & 251 & 262 & 1.05 \\
Annual & $\mathbf{1 , 0 5 4}$ & $\mathbf{1 0 8}$ & $\mathbf{3 5 0}$ & $\mathbf{2 2 3}$ & $\mathbf{0 . 6 4}$ \\
\hline
\end{tabular}

Magnitude and probability of annual low flow based on period of record, 1951-2004

\begin{tabular}{|c|c|c|c|c|c|c|}
\hline \multirow{3}{*}{$\begin{array}{l}\text { Period } \\
\text { (con- } \\
\text { secu- } \\
\text { tive } \\
\text { days) }\end{array}$} & \multicolumn{6}{|c|}{$\begin{array}{l}\text { Streamflow, in } \mathrm{ft}^{3} / \mathrm{s} \text {, for indicated recurrence interval, } \\
\text { in years, and non-exceedance probability, in percent }\end{array}$} \\
\hline & 2 & 5 & 10 & 20 & 50 & 100 \\
\hline & $\mathbf{5 0} \%$ & $20 \%$ & $10 \%$ & $5 \%$ & $2 \%$ & $1 \%$ \\
\hline 1 & 69 & 41 & 30 & 23 & 17 & 13 \\
\hline 2 & 71 & 42 & 31 & 24 & 17 & 13 \\
\hline 3 & 73 & 43 & 32 & 24 & 17 & 14 \\
\hline 7 & 78 & 48 & 36 & 28 & 20 & 16 \\
\hline 14 & 83 & 53 & 40 & 32 & 24 & 20 \\
\hline 30 & 94 & 60 & 47 & 37 & 29 & 24 \\
\hline 60 & 108 & 72 & 57 & 47 & 38 & 32 \\
\hline 90 & 116 & 77 & 62 & 52 & 42 & 36 \\
\hline 183 & 140 & 91 & 73 & 62 & 52 & 46 \\
\hline \multicolumn{7}{|c|}{ October-November } \\
\hline 1 & 89 & 57 & 45 & 36 & 29 & 25 \\
\hline 2 & 90 & 59 & 47 & 39 & 32 & 28 \\
\hline 3 & 92 & 60 & 48 & 41 & 33 & 29 \\
\hline 7 & 95 & 63 & 52 & 44 & 36 & 32 \\
\hline 14 & 103 & 69 & 56 & 47 & 39 & 35 \\
\hline 30 & 118 & 77 & 63 & 53 & 44 & 39 \\
\hline 60 & 138 & 87 & 73 & 65 & 58 & 55 \\
\hline
\end{tabular}

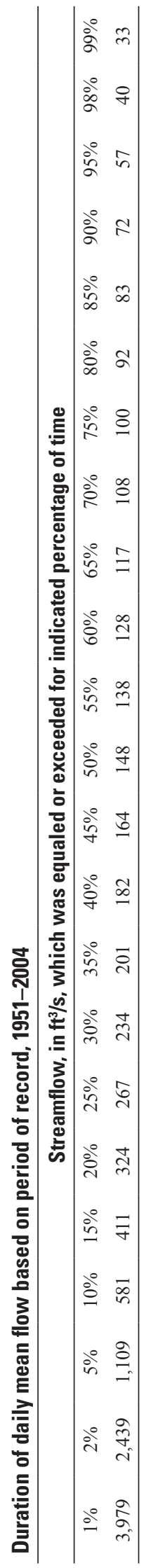




\section{WAHOO CREEK AT ASHLAND, NEBR.}

LOCATION.--Lat 41 $03^{\prime} 13^{\prime \prime}$, long 96 $22^{\prime} 04^{\prime \prime}$, in SW 1/4 NW 1/4 sec.36, T.1 3., R.9 E., Saunders County, Hydrologic Unit 10200203, at right upstream side of bridge near end of guard rail on State Highway 63, 1 mi north of Ashland, and at mile 2.6.

DRAINAGE AREA.--416 $\mathrm{mi}^{2}$.

\section{STREAMFLOW RECORDS}

REVISED RECORDS.--WDR NE-99-1: Datum.

PERIOD OF RECORD.--September 1990 to current year.

GAGE.--Water-stage recorder. Datum of gage is $1,048.77 \mathrm{ft}$ above sea level.

REMARKS.--Records good except for estimated daily streamflows, which are poor.

Non-exceedance duration hydrograph for Wahoo Cr at Ashland, Nebr. (06804700) (1990-2004 period of record)

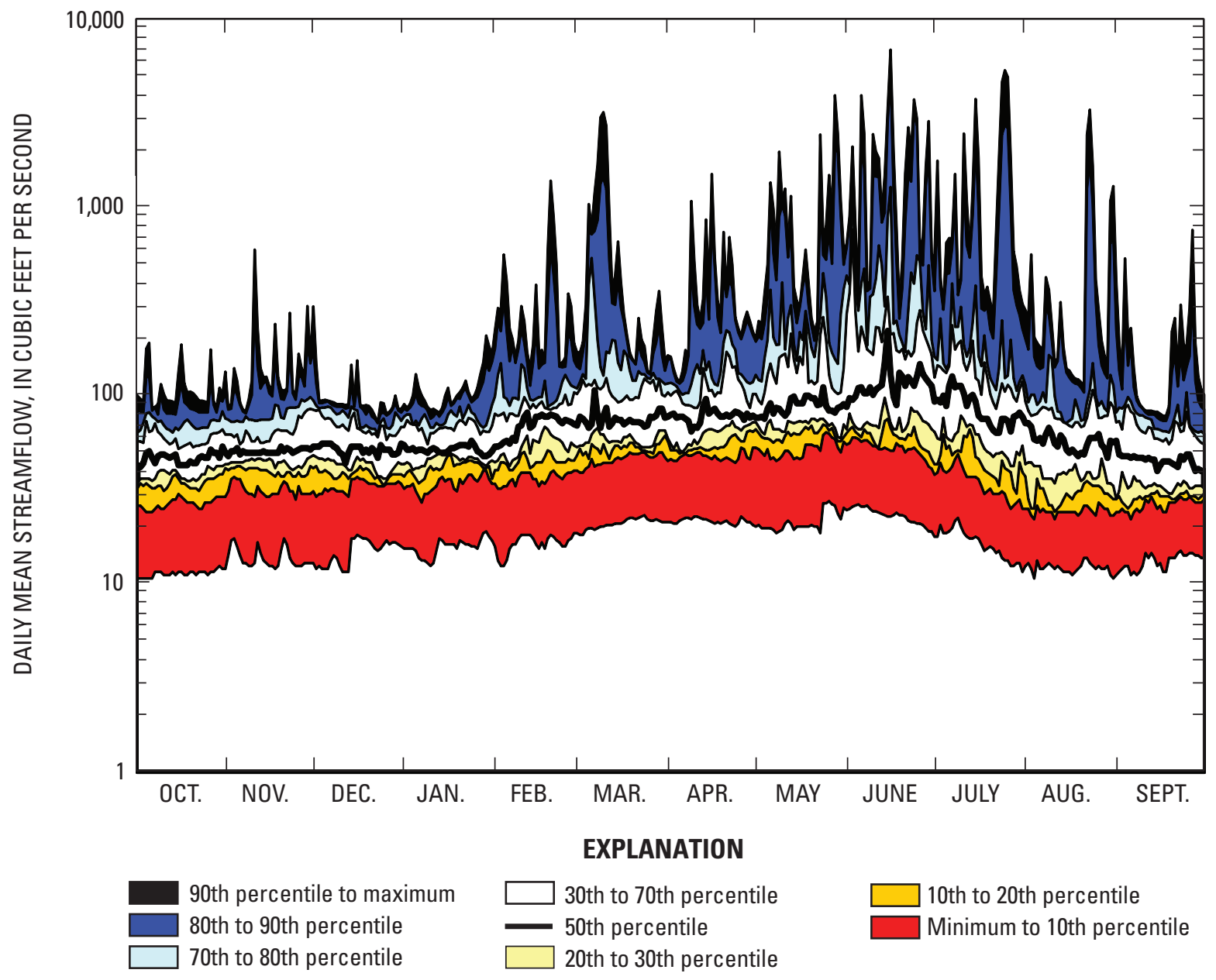


06804700 WAHOO CREEK AT ASHLAND, NEBR.

Monthly and annual streamflow, 1990-2004

\begin{tabular}{|c|c|c|c|c|c|c|}
\hline & \multicolumn{2}{|c|}{$\begin{array}{l}\text { Maximum } \\
\qquad\left(\mathrm{ft}^{3} / \mathrm{s}\right)\end{array}$} & $\begin{array}{l}\text { Mini- } \\
\text { mum } \\
\left(\mathrm{ft}^{3} / \mathrm{s}\right)\end{array}$ & $\begin{array}{l}\text { Mean } \\
\left(\mathrm{ft}^{3} / \mathrm{s}\right)\end{array}$ & $\begin{array}{c}\text { Stan- } \\
\text { dard } \\
\text { devia- } \\
\text { tion }\end{array}$ & $\begin{array}{l}\text { Coef- } \\
\text { ficient } \\
\text { of } \\
\text { varia- } \\
\text { tion }\end{array}$ \\
\hline October & \multicolumn{2}{|c|}{98} & 29 & 53 & 18 & 0.35 \\
\hline November & \multicolumn{2}{|c|}{125} & 36 & 62 & 25 & 0.41 \\
\hline December & \multicolumn{2}{|c|}{86} & 37 & 56 & 17 & 0.30 \\
\hline January & \multicolumn{2}{|c|}{92} & 36 & 58 & 19 & 0.32 \\
\hline February & \multicolumn{2}{|c|}{199} & 36 & 88 & 45 & 0.51 \\
\hline March & \multicolumn{2}{|c|}{581} & 47 & 130 & 136 & 1.05 \\
\hline April & \multicolumn{2}{|c|}{247} & 47 & 109 & 63 & 0.57 \\
\hline May & \multicolumn{2}{|c|}{552} & 68 & 187 & 154 & 0.82 \\
\hline June & \multicolumn{2}{|c|}{1,031} & 56 & 299 & 276 & 0.92 \\
\hline July & \multicolumn{2}{|c|}{1,032} & 34 & 179 & 246 & 1.37 \\
\hline August & \multicolumn{2}{|c|}{341} & 23 & 90 & 86 & 0.96 \\
\hline September & \multicolumn{2}{|c|}{150} & 28 & 55 & 32 & 0.58 \\
\hline Annual & \multicolumn{2}{|c|}{223} & 50 & 114 & 54 & 0.48 \\
\hline \multicolumn{7}{|c|}{$\begin{array}{l}\text { Magnitude and probability of annual low flow based on period } \\
\text { of record, } 1990-2004\end{array}$} \\
\hline \multirow{3}{*}{$\begin{array}{l}\text { Period } \\
\text { (con- } \\
\text { secu- } \\
\text { tive } \\
\text { days) } \\
\end{array}$} & \multicolumn{6}{|c|}{$\begin{array}{l}\text { Streamflow, in } \mathrm{ft}^{3} / \mathrm{s} \text {, for indicated recurrence interval, } \\
\text { in years, and non-exceedance probability, in percent }\end{array}$} \\
\hline & 2 & 5 & 10 & 20 & 50 & 100 \\
\hline & $50 \%$ & $20 \%$ & $10 \%$ & $5 \%$ & $2 \%$ & $1 \%$ \\
\hline 1 & 32 & 24 & 21 & 19 & 17 & 16 \\
\hline 2 & 33 & 25 & 22 & 19 & 17 & 16 \\
\hline 3 & 33 & 25 & 22 & 20 & 18 & 16 \\
\hline 7 & 35 & 27 & 23 & 21 & 19 & 18 \\
\hline 14 & 38 & 29 & 25 & 22 & 19 & 17 \\
\hline 30 & 43 & 31 & 27 & 23 & 20 & 18 \\
\hline 60 & 48 & 36 & 30 & 26 & 22 & 20 \\
\hline 90 & 49 & 37 & 32 & 28 & 25 & 22 \\
\hline 183 & 56 & 42 & 36 & 32 & 28 & 25 \\
\hline \multicolumn{7}{|c|}{ October-November } \\
\hline 1 & 40 & 29 & 24 & 21 & 18 & 16 \\
\hline 2 & 41 & 29 & 25 & 21 & 18 & 16 \\
\hline 3 & 42 & 30 & 25 & 22 & 18 & 16 \\
\hline 7 & 43 & 31 & 26 & 22 & 19 & 17 \\
\hline 14 & 45 & 33 & 27 & 24 & 20 & 18 \\
\hline 30 & 48 & 35 & 29 & 25 & 21 & 18 \\
\hline 60 & 53 & 38 & 32 & 28 & 23 & 21 \\
\hline
\end{tabular}

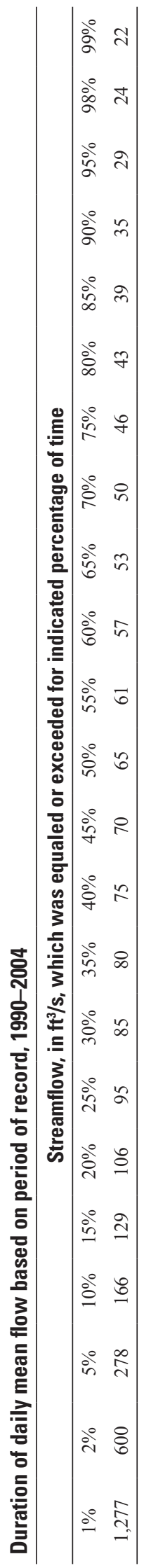




\section{PLATTE RIVER AT LOUISVILLE, NEBR.}

LOCATION.--Lat 41 $00^{\prime} 55^{\prime \prime}$, long 96092'8", in NW 1/4 NW 1/4 sec.14, T.12 N., R.11 E., Sarpy County, Hydrologic Unit 10200202, on the left bank at the upstream side of bridge on Nebraska Highway 50, 1 mi north of Louisville, and at mile 16.5.

DRAINAGE AREA.--85,329 $\mathrm{mi}^{2}$ of which $14,329 \mathrm{mi}^{2}$ probably is noncontributing.

\section{STREAMFLOW RECORDS}

REVISED RECORDS.--WDR NE-97-1: Drainage area; 1995.

PERIOD OF RECORD.--May 1953 to current year. October 1961 to September 1973 published as Platte River at South Bend.

GAGE.--Water-stage recorder. Datum of gage is 1,007.10 ft above sea level. Dec. 5, 1961 to Sept. 30, 1973 at site 7 mi upstream at datum $31.43 \mathrm{ft}$ higher. Data collection platform at station.

REMARKS.--Records good except for estimated daily streamflows, which are poor. Natural flow of stream affected by storage reservoirs, power developments, ground-water withdrawals and diversions for irrigation, and return flow from irrigated areas.

EXTREMES FOR PERIOD OF RECORD.--Maximum streamflow, 124,000 $\mathrm{ft}^{3}$ Mar. 30, 1960, gage height, 12.45 feet; minimum daily, $131 \mathrm{ft}^{3}$ Sept. 3 , 1976. Flood stage is 9.0 feet, gage datum.

Non-exceedance duration hydrograph for Platte River at Louisville, Nebr. (06805500) (1953-2004 period of record)

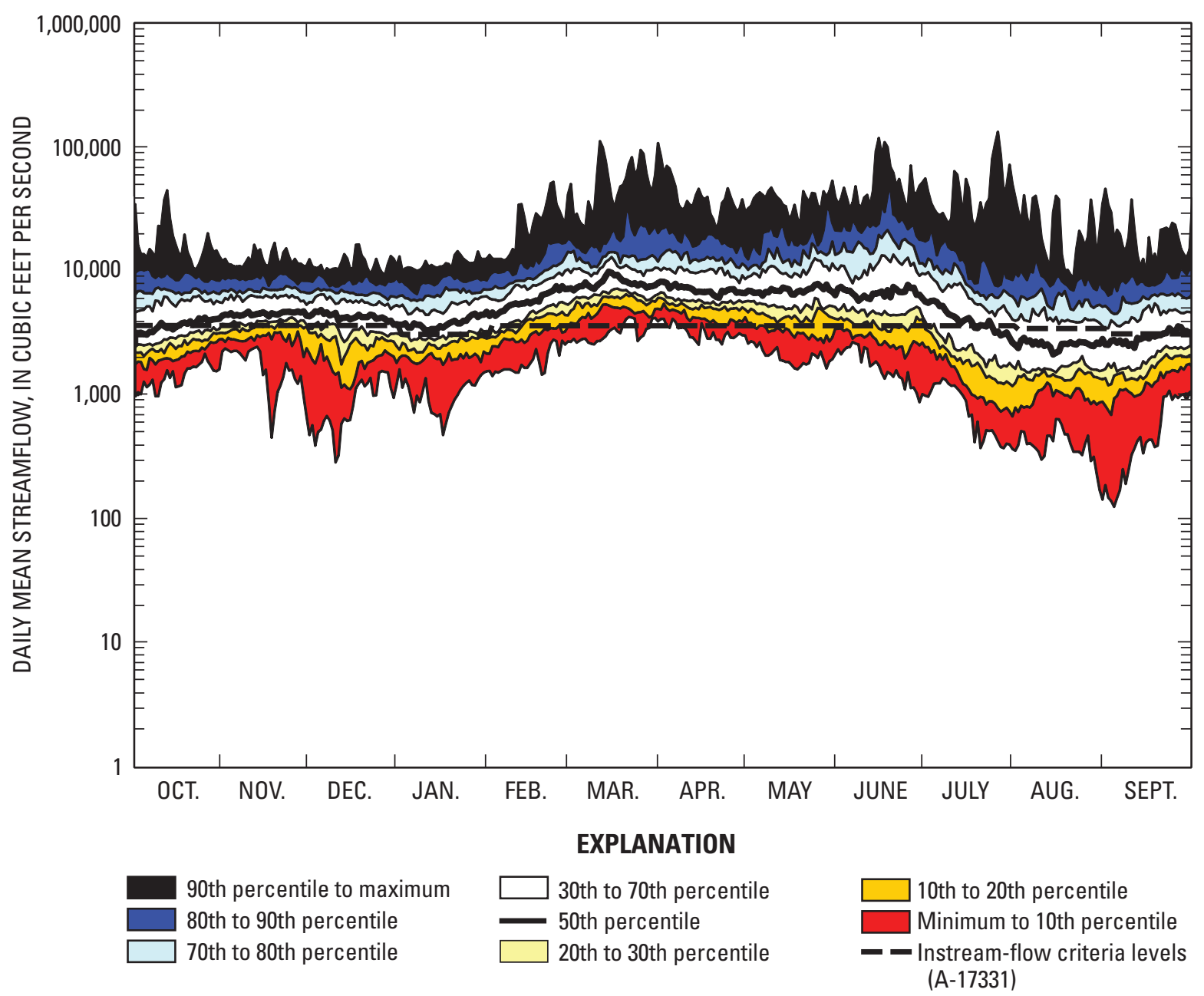


06805500 PLATTE RIVER AT LOUISVILLE, NEBR.

Monthly and annual streamflow, 1953-2005

\begin{tabular}{|c|c|c|c|c|c|}
\hline & $\begin{array}{c}\text { Maximum } \\
\qquad\left(\mathrm{ft}^{3} / \mathrm{s}\right)\end{array}$ & $\begin{array}{c}\text { Mini- } \\
\text { mum } \\
\left(\mathrm{ft}^{3} / \mathrm{s}\right)\end{array}$ & $\begin{array}{l}\text { Mean } \\
\left(\mathrm{ft}^{3} / \mathrm{s}\right)\end{array}$ & $\begin{array}{c}\text { Stan- } \\
\text { dard } \\
\text { devia- } \\
\text { tion }\end{array}$ & $\begin{array}{l}\text { Coef- } \\
\text { ficient } \\
\text { of } \\
\text { varia- } \\
\text { tion }\end{array}$ \\
\hline October & 15,630 & 1,604 & 5,064 & 2,998 & 0.59 \\
\hline November & 10,580 & 2,234 & 5,456 & 2,125 & 0.39 \\
\hline December & 10,910 & 1,456 & 4,870 & 2,020 & 0.41 \\
\hline January & 10,810 & 1,822 & 4,711 & 2,121 & 0.45 \\
\hline February & 17,270 & 3,237 & 7,441 & 3,184 & 0.43 \\
\hline March & 27,010 & 4,898 & 10,907 & 5,264 & 0.48 \\
\hline April & 34,250 & 3,701 & 9,782 & 5,736 & 0.59 \\
\hline May & 35,350 & 2,548 & 9,802 & 5,818 & 0.59 \\
\hline June & 39,430 & 2,493 & 11,075 & 8,175 & 0.74 \\
\hline July & 43,440 & 978 & 6,148 & 6,302 & 1.02 \\
\hline August & 13,890 & 519 & 4,029 & 3,027 & 0.75 \\
\hline September & 12,870 & 975 & 4,196 & 2,925 & 0.70 \\
\hline Annual & 16,210 & 2,885 & 6,967 & 2,947 & 0.42 \\
\hline
\end{tabular}

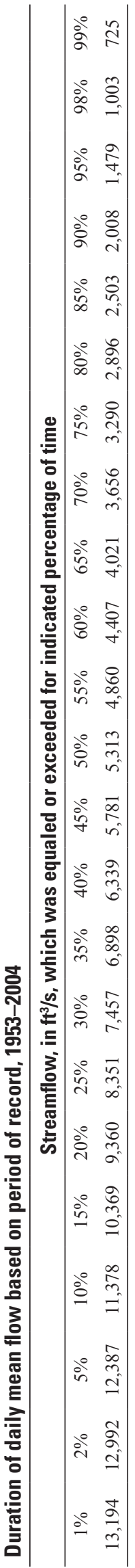

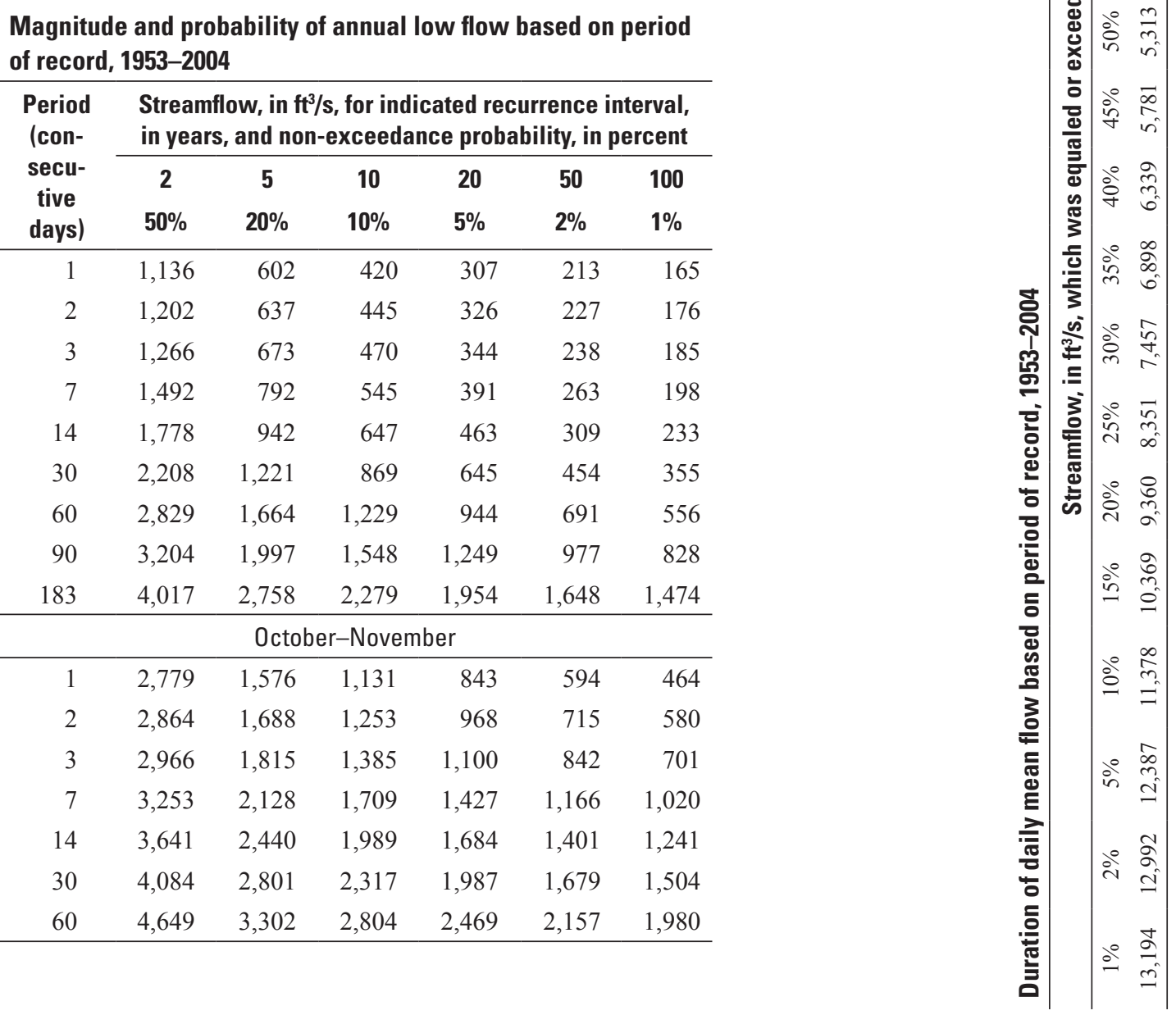





\section{Appendix 2. Trends in Streamflow Characteristics}


Table 2-1. Trends in streamflow of Platte River at Duncan, Nebraska (06774000).

[period analyzed is given as water years; p-value quantifies the probability of the available evidence to conclude for a trend slope different from zero could have arisen when there was actually no temporal trend; $\mathrm{ft}^{3} / \mathrm{s}$, cubic feet per second; $<$, less than; --, not significant]

\begin{tabular}{|c|c|c|c|c|}
\hline Streamflow statistic & Period analyzed & Kendall's tau & p-value & $\begin{array}{l}\text { Median slope of trend, in } \\
\mathrm{ft}^{3} / \mathrm{s} \text { per year }(\mathrm{p}<0.05)\end{array}$ \\
\hline \multicolumn{5}{|c|}{ Mean flow } \\
\hline October mean flow & $1942-2004$ & 0.108 & 0.103 & -- \\
\hline November mean flow & $1942-2004$ & .059 & .378 & -- \\
\hline December mean flow & $1942-2004$ & .135 & .042 & 18 \\
\hline January mean flow & $1942-2004$ & .161 & .016 & 18 \\
\hline February mean flow & $1942-2004$ & .065 & .331 & -- \\
\hline March mean flow & $1942-2004$ & .020 & .759 & -- \\
\hline April mean flow & $1942-2004$ & .023 & .732 & -- \\
\hline May mean flow & $1942-2004$ & .064 & .335 & -- \\
\hline June mean flow & $1942-2004$ & .024 & .721 & -- \\
\hline July mean flow & $1942-2004$ & .048 & .474 & -- \\
\hline August mean flow & $1942-2004$ & .148 & .026 & 21 \\
\hline September mean flow & $1942-2004$ & .131 & .049 & 20 \\
\hline Annual mean flow & 1929-2003 & .201 & .011 & 13 \\
\hline \multicolumn{5}{|c|}{ Low flow } \\
\hline 1-day low flow & 1930-2004 & .397 & $<.001$ & 1.1 \\
\hline 2-day low flow & 1930-2004 & .391 & $<.001$ & 1.2 \\
\hline 3-day low flow & 1930-2004 & .394 & $<.001$ & 1.3 \\
\hline 7-day low flow & 1930-2004 & .389 & $<.001$ & 1.6 \\
\hline 14-day low flow & 1930-2004 & .401 & $<.001$ & 2.5 \\
\hline 30-day low flow & 1930-2004 & .444 & $<.001$ & 4.8 \\
\hline 60-day low flow & 1930-2004 & .412 & $<.001$ & 7.5 \\
\hline 90-day low flow & 1930-2004 & .361 & $<.001$ & 11 \\
\hline 183-day low flow & 1930-2004 & .307 & $<.001$ & 12 \\
\hline 1-day low flow (Oct-Nov) & $1928-2003$ & .274 & $<.001$ & 8.2 \\
\hline 2-day low flow (Oct-Nov) & $1928-2003$ & .261 & .001 & 8.4 \\
\hline 3-day low flow (Oct-Nov) & $1928-2003$ & .260 & .001 & 8.7 \\
\hline 7-day low flow (Oct-Nov) & $1928-2003$ & .267 & .001 & 10 \\
\hline 14-day low flow (Oct-Nov) & $1928-2003$ & .270 & .001 & 12 \\
\hline 30-day low flow (Oct-Nov) & 1928-2003 & .244 & .002 & 13 \\
\hline 60-day low flow (Oct-Nov) & $1928-2003$ & .225 & .004 & 14 \\
\hline
\end{tabular}




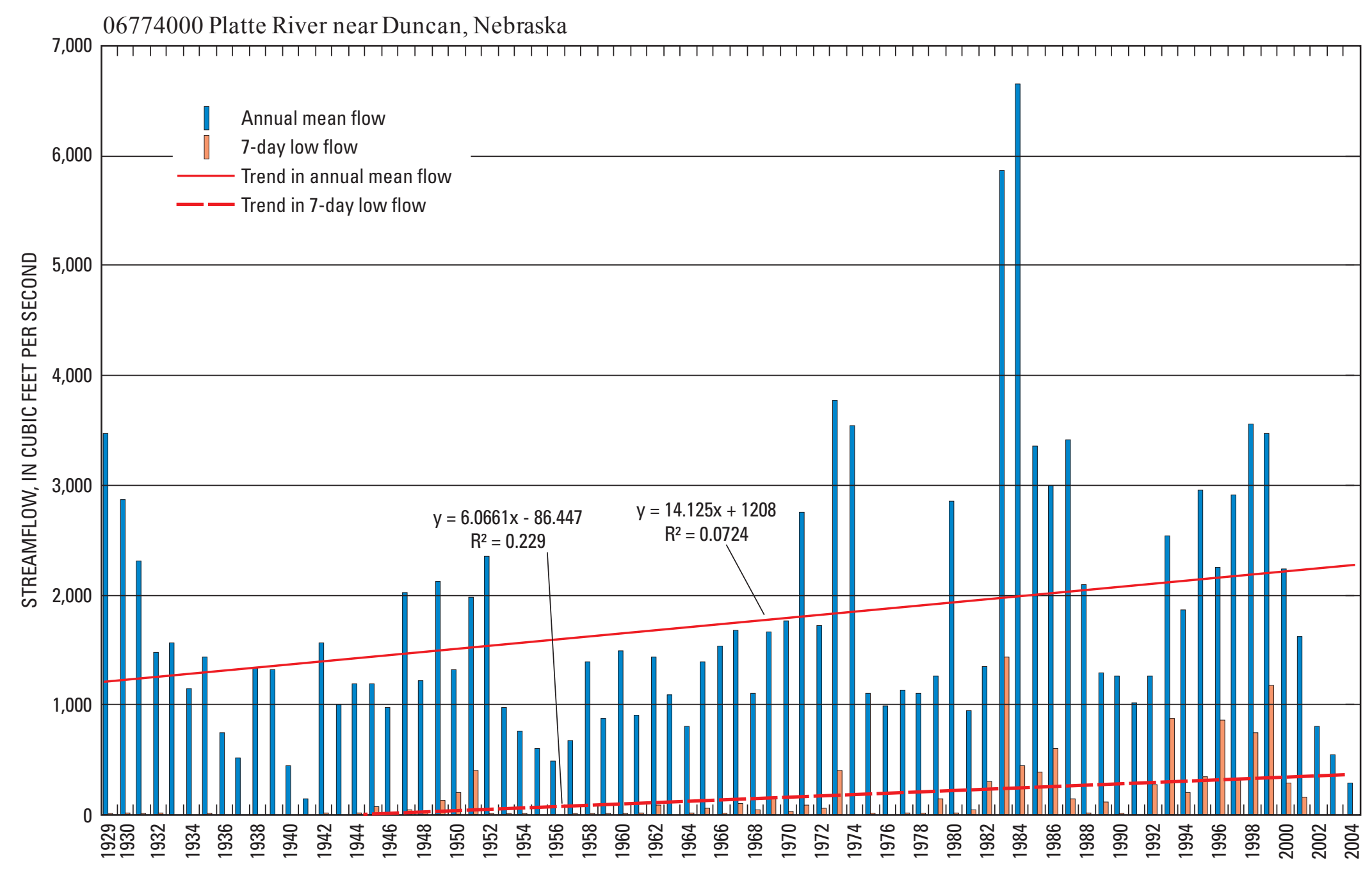

Figure 2-1. Annual mean flow, 7-day lowflow, trend in annual mean flow, and trend in 7-day low flow of Platte River near Duncan, Nebr. 
Table 2-2. Trends in streamflow of Platte River at North Bend, Nebraska (06796000).

[period analyzed is given as water years; p-value quantifies the probability of the available evidence to conclude for a trend slope different from zero could have arisen when there was actually no temporal trend; $\mathrm{ft}^{3} / \mathrm{s}$, cubic feet per second; <, less than; --, not significant]

\begin{tabular}{|c|c|c|c|c|}
\hline Streamflow statistic & Period analyzed & Kendall's tau & p-value & $\begin{array}{l}\text { Median slope of trend, } \\
\text { in } \mathrm{ft}^{3} / \mathrm{s} \text { per year }(\mathrm{p}<0.05)\end{array}$ \\
\hline \multicolumn{5}{|c|}{ Mean flow } \\
\hline October mean flow & 1949-2004 & 0.200 & 0.029 & 29 \\
\hline November mean flow & 1949-2004 & .190 & .038 & 27 \\
\hline December mean flow & 1949-2004 & .239 & .009 & 29 \\
\hline January mean flow & 1949-2004 & .176 & .055 & -- \\
\hline February mean flow & 1949-2004 & .032 & .729 & -- \\
\hline March mean flow & 1949-2004 & -.036 & .692 & -- \\
\hline April mean flow & 1949-2004 & .057 & .534 & -- \\
\hline May mean flow & 1949-2004 & .044 & .631 & -- \\
\hline June mean flow & 1949-2004 & .045 & .621 & -- \\
\hline July mean flow & 1949-2004 & .062 & .497 & -- \\
\hline August mean flow & 1949-2004 & .129 & .162 & -- \\
\hline September mean flow & 1949-2004 & .170 & .064 & -- \\
\hline Annual mean flow & 1949-2003 & .194 & .039 & 26 \\
\hline \multicolumn{5}{|c|}{ Low flow } \\
\hline 1-day low flow & $1950-2004$ & .104 & .264 & -- \\
\hline 2-day low flow & $1950-2004$ & .087 & .353 & -- \\
\hline 3-day low flow & $1950-2004$ & .099 & .289 & -- \\
\hline 7-day low flow & $1950-2004$ & .134 & .151 & -- \\
\hline 14-day low flow & $1950-2004$ & .156 & .095 & -- \\
\hline 30-day low flow & $1950-2004$ & .192 & .039 & 14 \\
\hline 60-day low flow & $1950-2004$ & .201 & .031 & 19 \\
\hline 90-day low flow & $1950-2004$ & .184 & .048 & 22 \\
\hline 183-day low flow & $1950-2004$ & .230 & .014 & 23 \\
\hline 1-day low flow (Oct-Nov) & $1948-2003$ & .198 & .033 & 18 \\
\hline 2-day low flow (Oct-Nov) & $1948-2003$ & .228 & .014 & 23 \\
\hline 3-day low flow (Oct-Nov) & $1948-2003$ & .230 & .014 & 24 \\
\hline 7-day low flow (Oct-Nov) & $1948-2003$ & .231 & .013 & 23 \\
\hline 14-day low flow (Oct-Nov) & $1948-2003$ & .236 & .011 & 25 \\
\hline 30-day low flow (Oct-Nov) & $1948-2003$ & .226 & .015 & 27 \\
\hline 60-day low flow (Oct-Nov) & $1948-2003$ & .235 & .012 & 30 \\
\hline
\end{tabular}


06796000 Platte River at North Bend, Nebraska

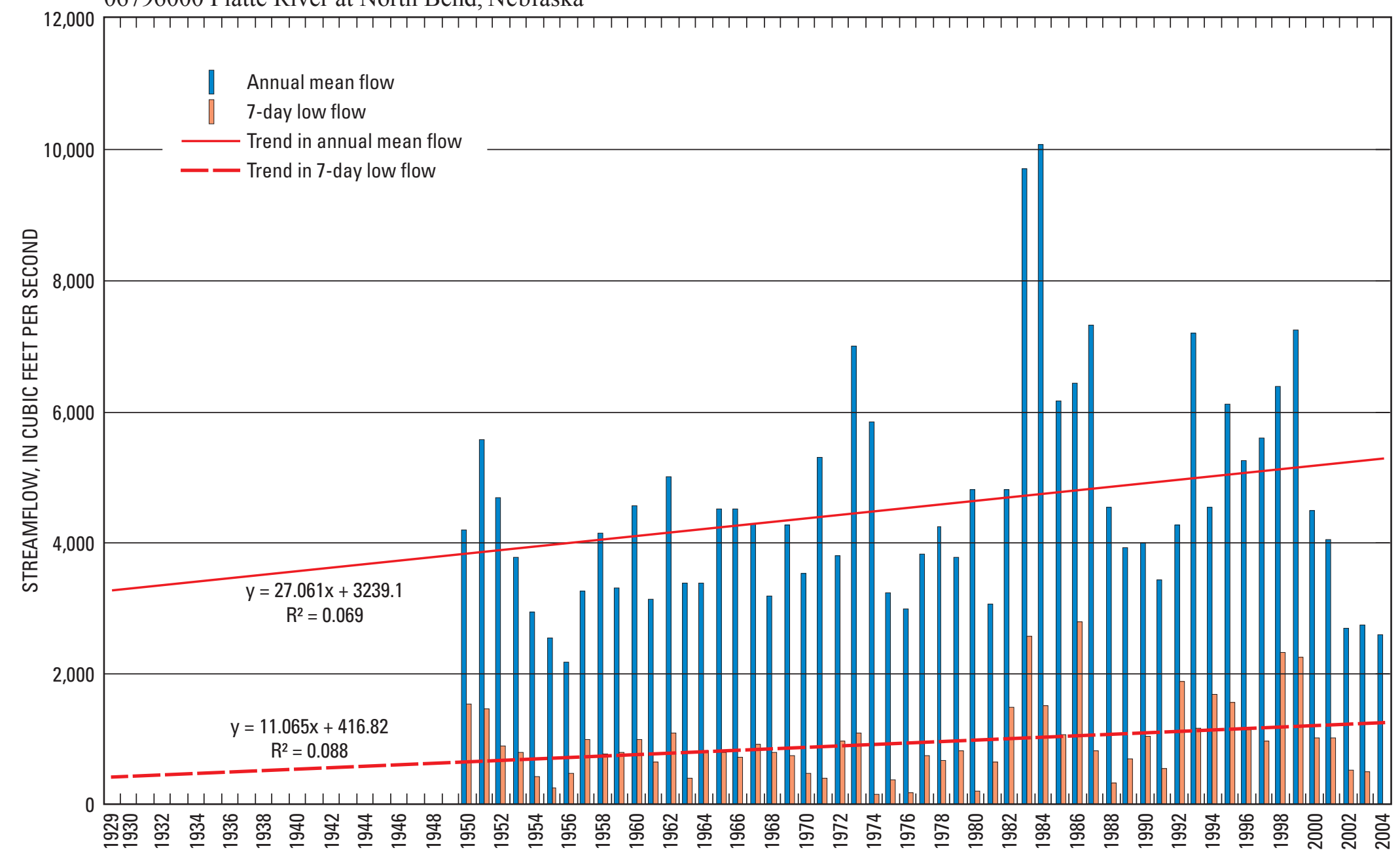

Figure 2-2. Annual mean flow, 7-day lowflow, trend in annual mean flow, and trend in 7-day low flow of Platte River at North Bend, Nebr. 
Table 2-3. Trends in streamflow of Platte River near Leshara, Nebraska (06796500).

[period analyzed is given as water years; p-value quantifies the probability of the available evidence to conclude for a trend slope different from zero could have arisen when there was actually no temporal trend; $\mathrm{ft}^{3} / \mathrm{s}$, cubic feet per second; <, less than; --, not significant]

\begin{tabular}{|c|c|c|c|c|}
\hline Streamflow statistic & Period analyzed & Kendall's tau & p-value & $\begin{array}{l}\text { Median slope of trend, } \\
\text { in } \mathrm{ft}^{3} / \mathrm{s} \text { per year }(\mathrm{p}<0.05)\end{array}$ \\
\hline \multicolumn{5}{|c|}{ Mean flow } \\
\hline October mean flow & 1995-2005 & -0.600 & 0.010 & -400 \\
\hline November mean flow & 1995-2005 & -.564 & .016 & -380 \\
\hline December mean flow & 1995-2005 & -.564 & .016 & -390 \\
\hline January mean flow & 1995-2005 & -.582 & .012 & -410 \\
\hline February mean flow & 1995-2005 & -.455 & .052 & -- \\
\hline March mean flow & 1995-2005 & -.273 & .243 & -- \\
\hline April mean flow & 1995-2005 & -.418 & .073 & -- \\
\hline May mean flow & 1995-2005 & -.418 & .073 & -- \\
\hline June mean flow & 1995-2005 & -.527 & .024 & $-1,100$ \\
\hline July mean flow & 1995-2005 & -.600 & .010 & -610 \\
\hline August mean flow & 1995-2005 & -.491 & .036 & -530 \\
\hline September mean flow & 1995-2005 & -.709 & .002 & -420 \\
\hline Annual mean flow & 1994-2004 & -.511 & .049 & -510 \\
\hline \multicolumn{5}{|c|}{ Low flow } \\
\hline 1-day low flow & $1995-2005$ & -.378 & .152 & -- \\
\hline 2-day low flow & 1995-2005 & -.378 & .152 & -- \\
\hline 3-day low flow & 1995-2005 & -.378 & .152 & -- \\
\hline 7-day low flow & 1995-2005 & -.467 & .074 & -- \\
\hline 14-day low flow & 1995-2005 & -.467 & .074 & -- \\
\hline 30-day low flow & 1995-2005 & -.467 & .074 & -- \\
\hline 60-day low flow & 1995-2005 & -.511 & .049 & -380 \\
\hline 90-day low flow & 1995-2005 & -.511 & .049 & -450 \\
\hline 183-day low flow & 1995-2005 & -.600 & .020 & -430 \\
\hline 1-day low flow (Oct-Nov) & 1994-2005 & -.491 & .043 & -280 \\
\hline 2-day low flow (Oct-Nov) & 1994-2005 & -.455 & .062 & -- \\
\hline 3-day low flow (Oct-Nov) & 1994-2005 & -.455 & .062 & -- \\
\hline 7-day low flow (Oct-Nov) & 1994-2005 & -.491 & .043 & -330 \\
\hline 14-day low flow (Oct-Nov) & 1994-2005 & -.527 & .029 & -350 \\
\hline 30-day low flow (Oct-Nov) & 1994-2005 & -.600 & .013 & -390 \\
\hline 60-day low flow (Oct-Nov) & 1994-2005 & -.673 & .005 & -450 \\
\hline
\end{tabular}




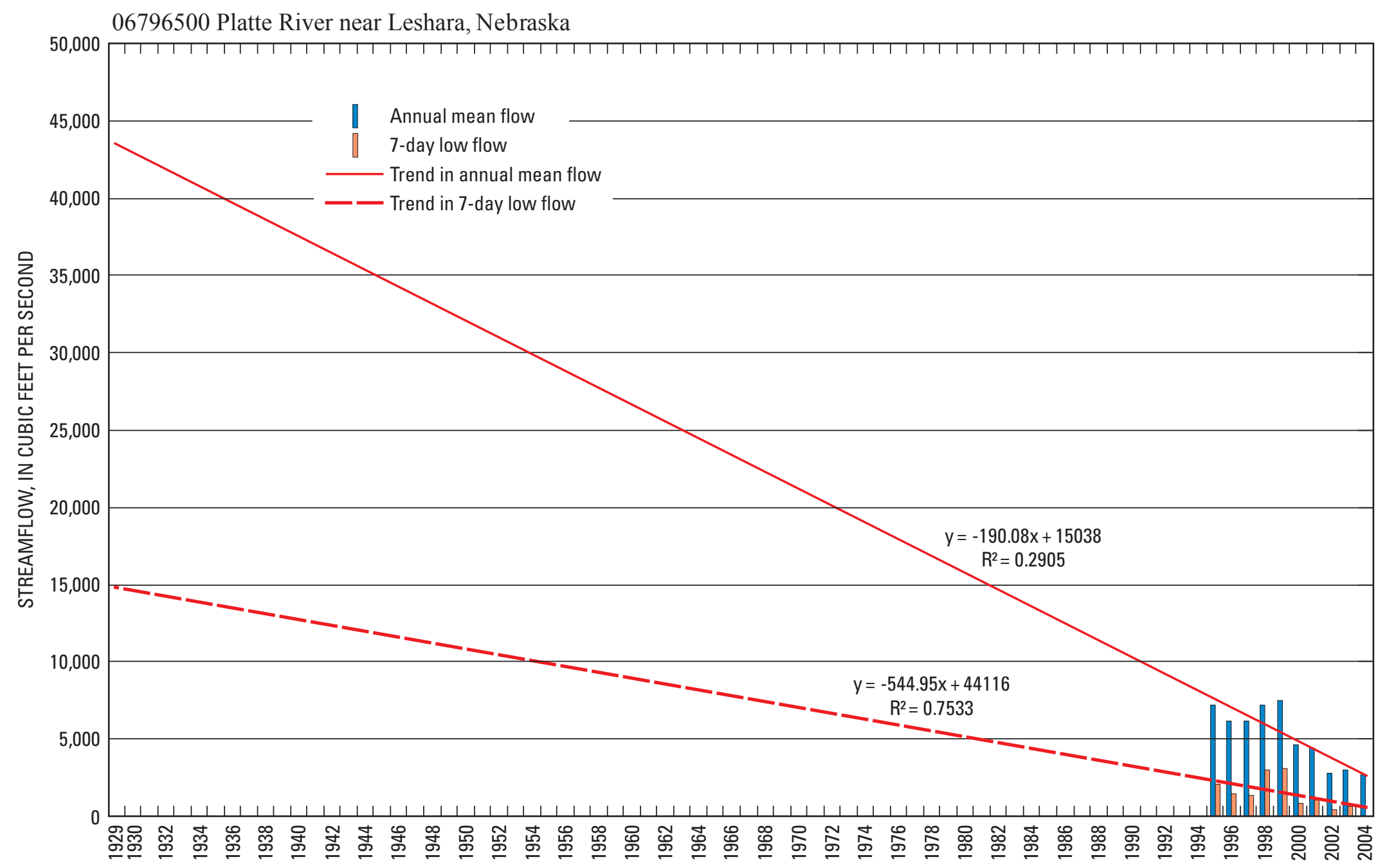

Figure 2-3. Annual mean flow, 7-day lowflow, trend in annual mean flow, and trend in 7-day low flow of Platte River near Leshara, Nebr. 
Table 2-4. Trends in streamflow of Elkhorn River near Atkinson, Nebraska (06796973).

[period analyzed is given as water years; p-value quantifies the probability of the available evidence to conclude for a trend slope different from zero could have arisen when there was actually no temporal trend; $\mathrm{ft}^{3} / \mathrm{s}$, cubic feet per second; <, less than; --, not significant]

\begin{tabular}{|c|c|c|c|c|}
\hline Streamflow statistic & Period analyzed & Kendall's tau & p-value & $\begin{array}{l}\text { Median slope of trend, } \\
\text { in } \mathrm{ft}^{3} / \mathrm{s} \text { per year }(\mathrm{p}<0.05)\end{array}$ \\
\hline \multicolumn{5}{|c|}{ Mean flow } \\
\hline October mean flow & 1983-2004 & -0.600 & 0.010 & -1.2 \\
\hline November mean flow & 1983-2004 & -.564 & .016 & 0 \\
\hline December mean flow & 1983-2004 & -.564 & .016 & -0.2 \\
\hline January mean flow & 1983-2004 & -.582 & .012 & -.6 \\
\hline February mean flow & 1983-2004 & -.455 & .052 & -- \\
\hline March mean flow & 1983-2004 & -.273 & .243 & -- \\
\hline April mean flow & 1983-2004 & -.418 & .073 & -- \\
\hline May mean flow & 1983-2004 & -.418 & .073 & -- \\
\hline June mean flow & 1983-2004 & -.527 & .024 & 1.0 \\
\hline July mean flow & 1983-2004 & -.600 & .010 & -3.5 \\
\hline August mean flow & 1983-2004 & -.491 & .036 & -1.3 \\
\hline September mean flow & 1983-2004 & -.709 & .002 & -4.2 \\
\hline Annual mean flow & $1983-2003$ & -.158 & .347 & -- \\
\hline \multicolumn{5}{|c|}{ Low flow } \\
\hline 1-day low flow & 1984-2004 & -.195 & .242 & -- \\
\hline 2-day low flow & 1984-2004 & -.200 & .230 & -- \\
\hline 3-day low flow & 1984-2004 & -.200 & .230 & -- \\
\hline 7-day low flow & 1984-2004 & -.189 & .256 & -- \\
\hline 14-day low flow & 1984-2004 & -.179 & .284 & -- \\
\hline 30-day low flow & 1984-2004 & -.200 & .230 & -- \\
\hline 60-day low flow & 1984-2004 & -.221 & .183 & -- \\
\hline 90-day low flow & 1984-2004 & -.221 & .183 & -- \\
\hline 183-day low flow & 1984-2004 & -.200 & .230 & -- \\
\hline 1-day low flow (Oct-Nov) & $1982-2003$ & -.157 & .333 & -- \\
\hline 2-day low flow (Oct-Nov) & $1982-2003$ & -.157 & .334 & -- \\
\hline 3-day low flow (Oct-Nov) & $1982-2003$ & -.157 & .334 & -- \\
\hline 7-day low flow (Oct-Nov) & $1982-2003$ & -.152 & .349 & -- \\
\hline 14-day low flow (Oct-Nov) & $1982-2003$ & -.152 & .349 & -- \\
\hline 30-day low flow (Oct-Nov) & $1982-2003$ & -.152 & .349 & -- \\
\hline 60-day low flow (Oct-Nov) & $1982-2003$ & -.200 & 0.216 & -- \\
\hline
\end{tabular}


06796973 Elkhorn River near Atkinson, Nebraska

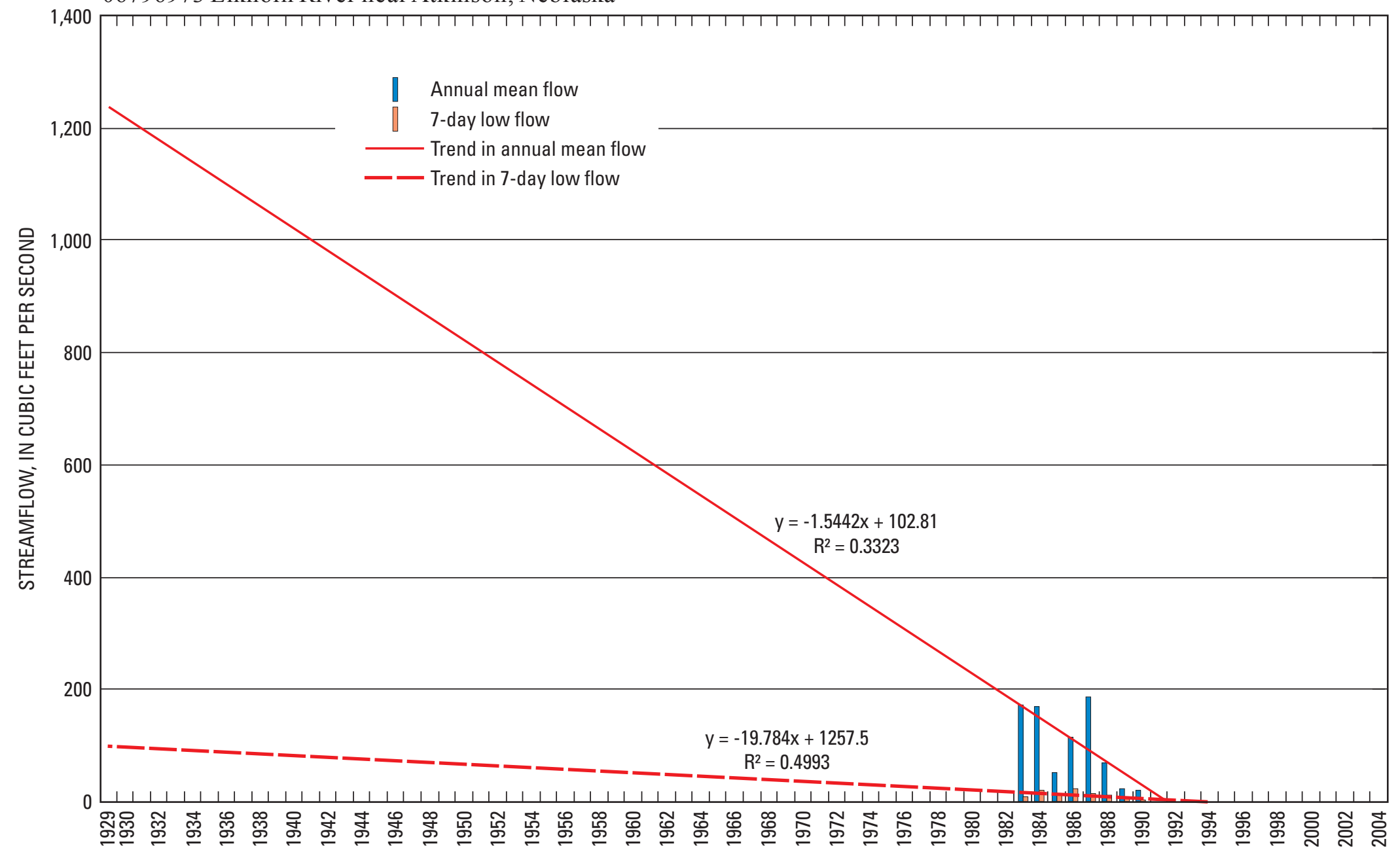

Figure 2-4. Annual mean flow, 7-day lowflow, trend in annual mean flow, and trend in 7-day low flow of Elkhorn River near Atkinson, Nebr. 
Table 2-5. Trends in streamflow of Elkhorn River at Ewing, Nebraska (06797500).

[period analyzed is given as water years; p-value quantifies the probability of the available evidence to conclude for a trend slope different from zero could have arisen when there was actually no temporal trend; $\mathrm{ft}^{3} / \mathrm{s}$, cubic feet per second; <, less than; --, not significant]

\begin{tabular}{|c|c|c|c|c|}
\hline Streamflow statistic & Period analyzed & Kendall's tau & p-value & $\begin{array}{l}\text { Median slope of trend, } \\
\text { in } \mathrm{ft}^{3} / \text { s per year }(\mathrm{p}<0.05)\end{array}$ \\
\hline \multicolumn{5}{|c|}{ Mean flow } \\
\hline October mean flow & $1948-2004$ & 0.014 & 0.874 & -- \\
\hline November mean flow & $1948-2004$ & .081 & .374 & -- \\
\hline December mean flow & 1948-2004 & .100 & .273 & -- \\
\hline January mean flow & $1948-2004$ & .150 & .100 & -- \\
\hline February mean flow & $1948-2004$ & .150 & .098 & -- \\
\hline March mean flow & $1948-2004$ & .023 & .799 & -- \\
\hline April mean flow & $1948-2004$ & -.009 & .923 & -- \\
\hline May mean flow & $1948-2004$ & .050 & .582 & -- \\
\hline June mean flow & 1948-2004 & -.048 & .596 & -- \\
\hline July mean flow & $1948-2004$ & -.025 & .783 & -- \\
\hline August mean flow & 1948-2004 & -.100 & .274 & -- \\
\hline September mean flow & 1948-2004 & -.053 & .558 & -- \\
\hline Annual mean flow & $1947-2003$ & .091 & .326 & -- \\
\hline \multicolumn{5}{|c|}{ Low flow } \\
\hline 1-day low flow & $1948-2004$ & -.072 & .437 & -- \\
\hline 2-day low flow & $1948-2004$ & -.077 & .404 & -- \\
\hline 3-day low flow & 1948-2004 & -.069 & .458 & -- \\
\hline 7-day low flow & $1948-2004$ & -.064 & .493 & -- \\
\hline 14-day low flow & $1948-2004$ & -.077 & .408 & -- \\
\hline 30-day low flow & $1948-2004$ & -.068 & .462 & -- \\
\hline 60-day low flow & 1948-2004 & -.053 & .572 & -- \\
\hline 90-day low flow & $1948-2004$ & -.023 & .805 & -- \\
\hline 183-day low flow & $1948-2004$ & .032 & .729 & -- \\
\hline 1-day low flow (Oct-Nov) & $1946-2003$ & .018 & .852 & -- \\
\hline 2-day low flow (Oct-Nov) & $1946-2003$ & .018 & .853 & -- \\
\hline 3-day low flow (Oct-Nov) & $1946-2003$ & .029 & .751 & -- \\
\hline 7-day low flow (Oct-Nov) & $1946-2003$ & .061 & .504 & -- \\
\hline 14-day low flow (Oct-Nov) & $1946-2003$ & .038 & .680 & -- \\
\hline 30-day low flow (Oct-Nov) & $1946-2003$ & .028 & .762 & -- \\
\hline 60-day low flow (Oct-Nov) & $1946-2003$ & .033 & .720 & -- \\
\hline
\end{tabular}




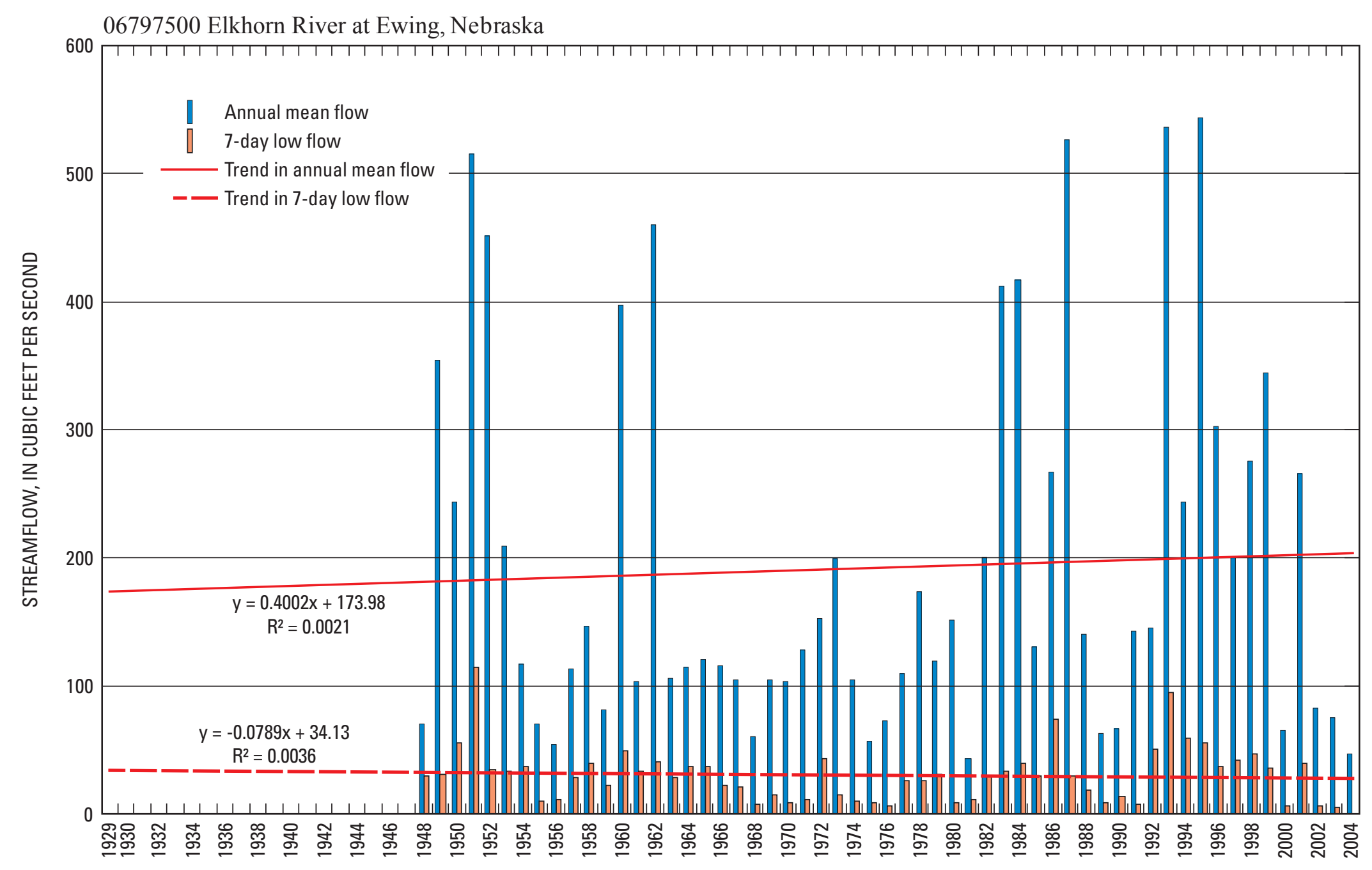

Figure 2-5. Annual mean flow, 7-day lowflow, trend in annual mean flow, and trend in 7-day low flow of Elkhorn River at Ewing, Nebr. 
Table 2-6. Trends in streamflow of South Fork Elkhorn River at Ewing, Nebraska (06798000).

[period analyzed is given as water years; p-value quantifies the probability of the available evidence to conclude for a trend slope different from zero could have arisen when there was actually no temporal trend; $\mathrm{ft}^{3} / \mathrm{s}$, cubic feet per second; <, less than; --, not significant]

\begin{tabular}{|c|c|c|c|c|}
\hline Streamflow statistic & Period analyzed & Kendall's tau & p-value & $\begin{array}{l}\text { Median slope of trend, } \\
\text { in } \mathrm{ft}^{3} / \mathrm{s} \text { per year }(\mathrm{p}<0.05)\end{array}$ \\
\hline \multicolumn{5}{|c|}{ Mean flow } \\
\hline October mean flow & $1948-2004$ & 0.088 & 0.395 & -- \\
\hline November mean flow & $1948-2004$ & -.078 & .451 & -- \\
\hline December mean flow & $1948-2004$ & .052 & .618 & -- \\
\hline January mean flow & $1948-2004$ & .344 & .001 & 0.4 \\
\hline February mean flow & $1948-2004$ & .064 & .538 & -- \\
\hline March mean flow & $1948-2004$ & -.358 & .001 & -2.0 \\
\hline April mean flow & $1948-2004$ & -.208 & .044 & -1.5 \\
\hline May mean flow & $1948-2004$ & -.043 & .674 & -- \\
\hline June mean flow & $1948-2004$ & .099 & .338 & -- \\
\hline July mean flow & $1948-2004$ & .224 & .030 & 2.1 \\
\hline August mean flow & $1948-2004$ & .197 & .053 & -- \\
\hline September mean flow & $1948-2004$ & .091 & .373 & -- \\
\hline Annual mean flow & $1947-2003$ & -.012 & .917 & -- \\
\hline \multicolumn{5}{|c|}{ Low flow } \\
\hline 1-day low flow & 1948-2004 & .290 & .009 & .2 \\
\hline 2-day low flow & $1948-2004$ & .263 & .017 & .2 \\
\hline 3-day low flow & $1948-2004$ & .235 & .034 & .1 \\
\hline 7-day low flow & $1948-2004$ & .219 & .047 & .1 \\
\hline 14-day low flow & $1948-2004$ & .178 & .108 & -- \\
\hline 30-day low flow & $1948-2004$ & .118 & .289 & -- \\
\hline 60-day low flow & 1948-2004 & .108 & .334 & -- \\
\hline 90-day low flow & $1948-2004$ & .095 & .395 & -- \\
\hline 183-day low flow & $1948-2004$ & .100 & .370 & -- \\
\hline 1-day low flow (Oct-Nov) & $1946-2003$ & .210 & .043 & .2 \\
\hline 2-day low flow (Oct-Nov) & $1946-2003$ & .217 & .036 & .2 \\
\hline 3-day low flow (Oct-Nov) & $1946-2003$ & .214 & .039 & .1 \\
\hline 7-day low flow (Oct-Nov) & $1946-2003$ & .192 & .064 & -- \\
\hline 14-day low flow (Oct-Nov) & $1946-2003$ & .123 & .237 & -- \\
\hline 30-day low flow (Oct-Nov) & $1946-2003$ & .127 & .221 & -- \\
\hline 60-day low flow (Oct-Nov) & $1946-2003$ & .098 & .348 & -- \\
\hline
\end{tabular}




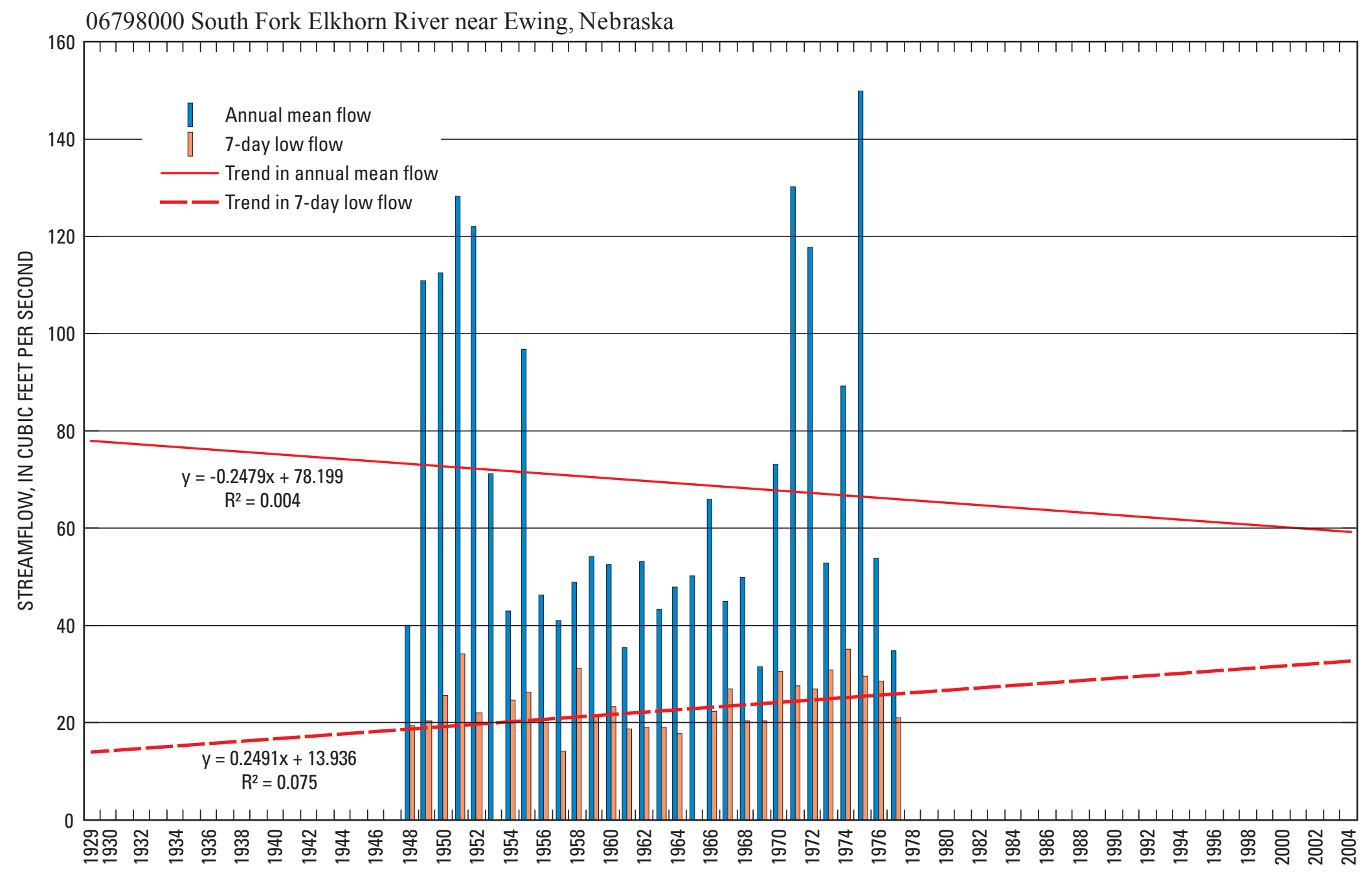

Figure 2-6. Annual mean flow, 7-day lowflow, trend in annual mean flow, and trend in 7-day low flow of South Fork Elkhorn River near Ewing, Nebr. 
Table 2-7. Trends in streamflow of Elkhorn River at Neligh, Nebraska (06798500).

[period analyzed is given as water years; p-value quantifies the probability of the available evidence to conclude for a trend slope different from zero could have arisen when there was actually no temporal trend; $\mathrm{ft}^{3} / \mathrm{s}$, cubic feet per second; $<$, less than; --, not significant]

\begin{tabular}{|c|c|c|c|c|}
\hline Streamflow statistic & Period analyzed & Kendall's tau & p-value & $\begin{array}{l}\text { Median slope of trend, } \\
\text { in } \mathrm{ft}^{3} / \mathrm{s} \text { per year }(\mathrm{p}<0.05)\end{array}$ \\
\hline \multicolumn{5}{|c|}{ Mean flow } \\
\hline October mean flow & $1931-2002$ & 0.346 & $<0.001$ & 0.9 \\
\hline November mean flow & $1931-2002$ & .444 & $<.001$ & 1.5 \\
\hline December mean flow & $1931-2002$ & .498 & $<.001$ & 1.1 \\
\hline January mean flow & $1931-2002$ & .473 & $<.001$ & 1.3 \\
\hline February mean flow & $1931-2002$ & .225 & .009 & 3.0 \\
\hline March mean flow & $1931-2002$ & .101 & .240 & -- \\
\hline April mean flow & $1931-2002$ & .287 & .001 & 9.7 \\
\hline May mean flow & $1931-2002$ & .300 & .001 & 7.4 \\
\hline June mean flow & $1931-2002$ & .182 & .035 & 1.4 \\
\hline July mean flow & $1931-2002$ & .214 & .013 & 3.1 \\
\hline August mean flow & $1931-2002$ & .162 & .061 & -- \\
\hline September mean flow & $1931-2002$ & .282 & .001 & 1.4 \\
\hline Annual mean flow & $1931-2003$ & .234 & .004 & 2.6 \\
\hline \multicolumn{5}{|c|}{ Low flow } \\
\hline 1-day low flow & $1932-2004$ & .178 & .032 & .5 \\
\hline 2-day low flow & $1932-2004$ & .172 & .038 & .5 \\
\hline 3-day low flow & $1932-2004$ & .172 & .039 & .5 \\
\hline 7-day low flow & $1932-2004$ & .191 & .022 & .6 \\
\hline 14-day low flow & $1932-2004$ & .158 & .058 & -- \\
\hline 30-day low flow & $1932-2004$ & .162 & .051 & -- \\
\hline 60-day low flow & $1932-2004$ & .178 & .032 & 6 \\
\hline 90-day low flow & $1932-2004$ & .177 & .033 & .7 \\
\hline 183-day low flow & $1932-2004$ & .208 & .012 & .8 \\
\hline 1-day low flow (Oct-Nov) & $1931-2004$ & .213 & .009 & 2.2 \\
\hline 2-day low flow (Oct-Nov) & $1930-2003$ & .129 & .112 & -- \\
\hline 3-day low flow (Oct-Nov) & $1930-2003$ & .140 & .084 & -- \\
\hline 7-day low flow (Oct-Nov) & $1930-2003$ & .140 & .085 & -- \\
\hline 14-day low flow (Oct-Nov) & $1930-2003$ & .144 & .077 & -- \\
\hline 30-day low flow (Oct-Nov) & $1930-2003$ & .157 & .053 & -- \\
\hline 60-day low flow (Oct-Nov) & $1930-2003$ & .188 & .021 & .7 \\
\hline
\end{tabular}


06798500 Elkhorn River at Neligh, Nebraska

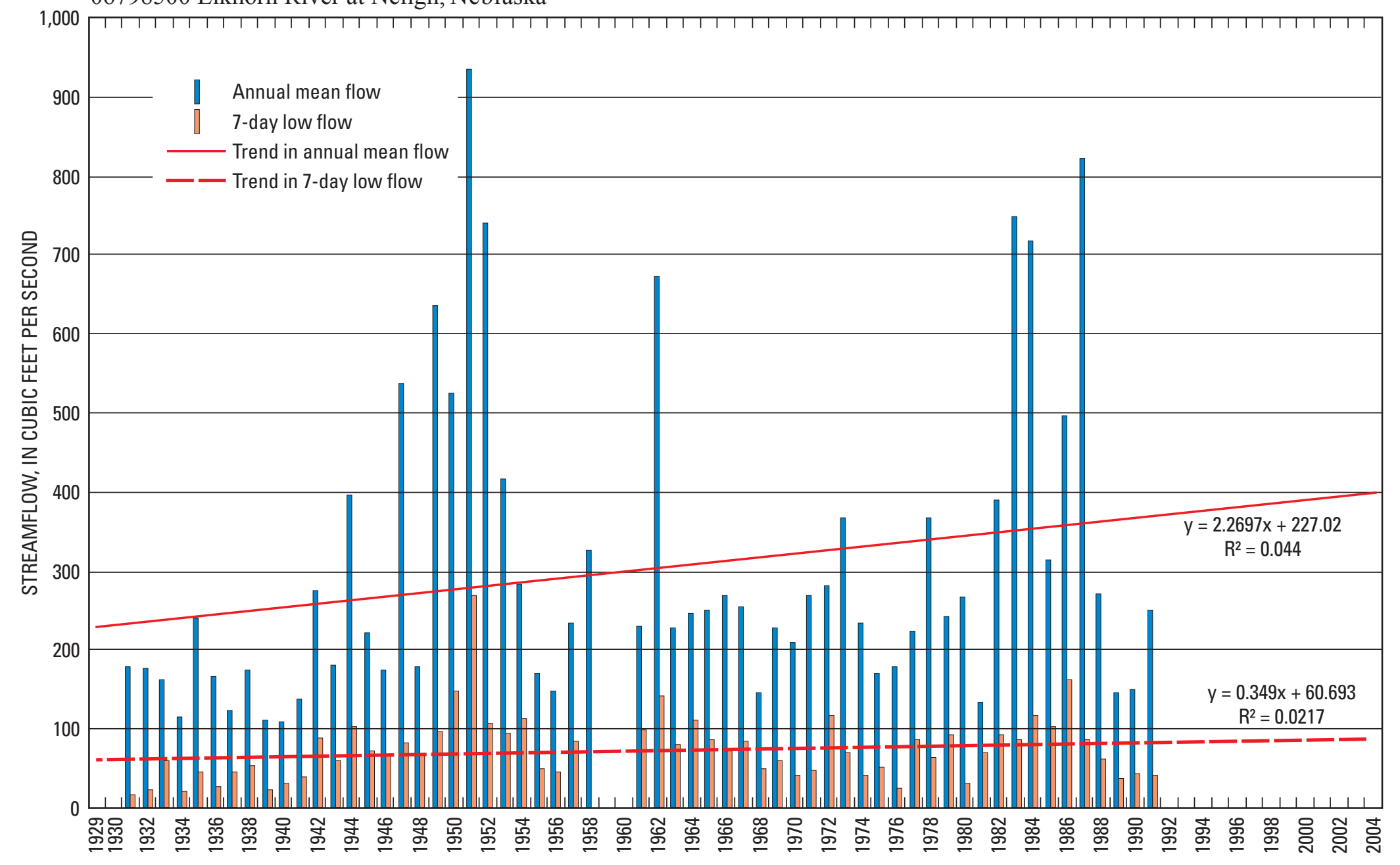

Figure 2-7. Annual mean flow, 7-day lowflow, trend in annual mean flow, and trend in 7-day low flow of Elkhorn River at Neligh, Nebr. 
Table 2-8. Trends in streamflow of Elkhorn River at Norfolk, Nebraska (06799000).

[period analyzed is given as water years; $p$-value quantifies the probability of the available evidence to conclude for a trend slope different from zero could have arisen when there was actually no temporal trend; $\mathrm{ft}^{3} / \mathrm{s}$, cubic feet per second; <, less than; --, not significant]

\begin{tabular}{|c|c|c|c|c|}
\hline Streamflow statistic & Period analyzed & Kendall's tau & p-value & $\begin{array}{l}\text { Median slope of trend, } \\
\text { in } \mathrm{ft}^{3} / \mathrm{s} \text { per year }(\mathrm{p}<0.05)\end{array}$ \\
\hline \multicolumn{5}{|c|}{ Mean flow } \\
\hline October mean flow & 1946-2004 & 0.037 & 0.676 & -- \\
\hline November mean flow & 1946-2004 & .124 & .164 & -- \\
\hline December mean flow & $1946-2004$ & .185 & .038 & 1.6 \\
\hline January mean flow & $1946-2004$ & .157 & .080 & -- \\
\hline February mean flow & $1946-2004$ & .103 & .250 & -- \\
\hline March mean flow & $1946-2004$ & -.016 & .860 & -- \\
\hline April mean flow & 1946-2004 & .021 & .814 & -- \\
\hline May mean flow & 1946-2004 & .106 & .234 & -- \\
\hline June mean flow & $1946-2004$ & -.043 & .633 & -- \\
\hline July mean flow & 1946-2004 & .014 & .875 & -- \\
\hline August mean flow & $1946-2004$ & -.020 & .824 & -- \\
\hline September mean flow & 1946-2004 & -.034 & .700 & -- \\
\hline Annual mean flow & $1896-2003$ & .066 & .464 & -- \\
\hline \multicolumn{5}{|c|}{ Low flow } \\
\hline 1-day low flow & $1897-2004$ & -.004 & .968 & -- \\
\hline 2-day low flow & $1897-2004$ & -.005 & .957 & -- \\
\hline 3-day low flow & $1897-2004$ & .004 & .968 & -- \\
\hline 7-day low flow & $1897-2004$ & -.027 & .773 & -- \\
\hline 14-day low flow & $1897-2004$ & -.041 & .658 & -- \\
\hline 30-day low flow & $1897-2004$ & -.026 & .778 & -- \\
\hline 60-day low flow & $1897-2004$ & .048 & .601 & -- \\
\hline 90-day low flow & $1897-2004$ & .056 & .537 & -- \\
\hline 183-day low flow & $1897-2004$ & .049 & .592 & -- \\
\hline 1-day low flow (Oct-Nov) & $1895-2003$ & .045 & .606 & -- \\
\hline 2-day low flow (Oct-Nov) & $1895-2003$ & .042 & .631 & -- \\
\hline 3-day low flow (Oct-Nov) & $1895-2003$ & .050 & .572 & -- \\
\hline 7-day low flow (Oct-Nov) & $1895-2003$ & .039 & .662 & -- \\
\hline 14-day low flow (Oct-Nov) & $1895-2003$ & .032 & .716 & -- \\
\hline 30-day low flow (Oct-Nov) & $1895-2003$ & .024 & .785 & -- \\
\hline 60-day low flow (Oct-Nov) & $1895-2003$ & .063 & .474 & -- \\
\hline
\end{tabular}




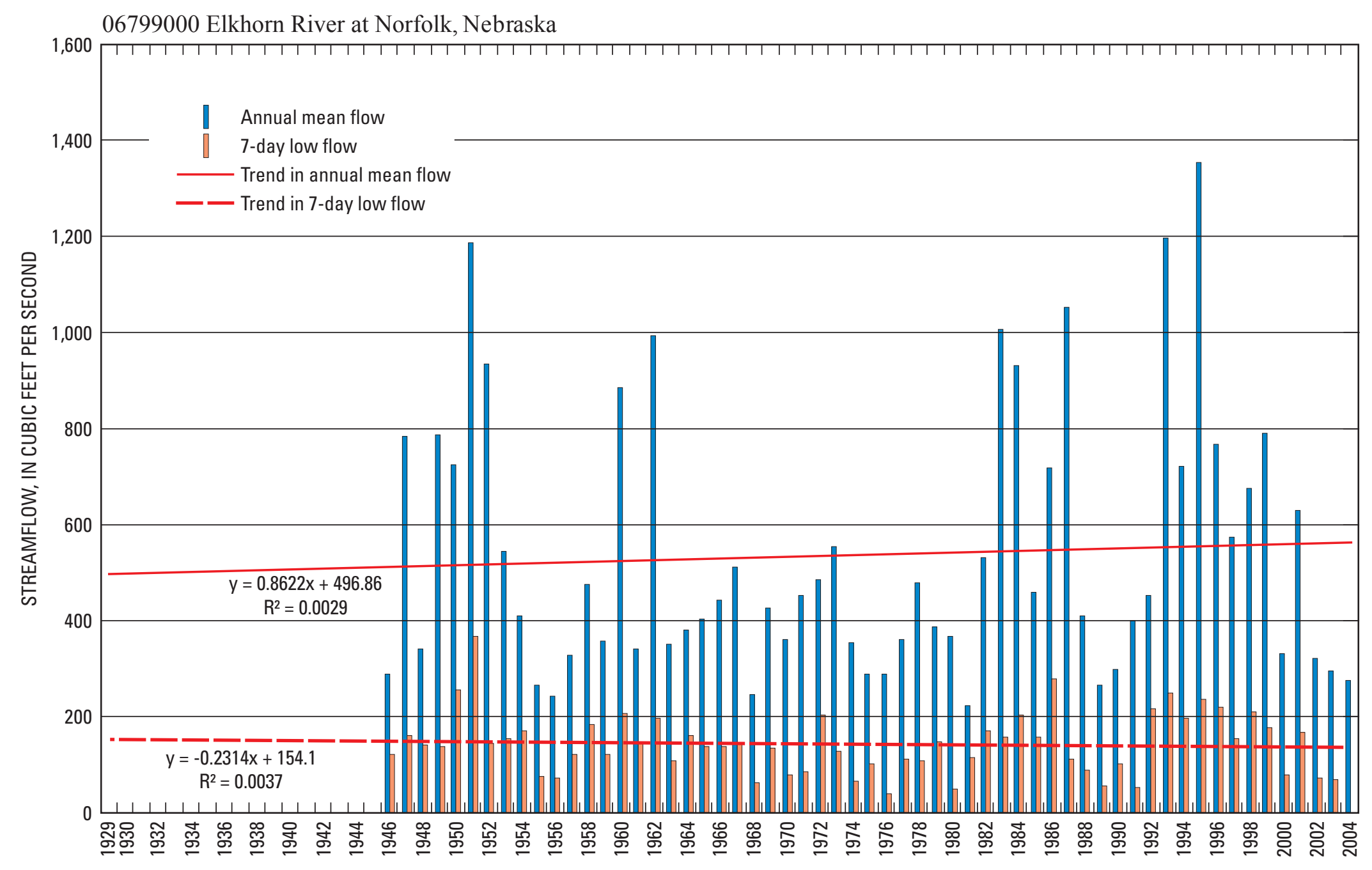

Figure 2-8. Annual mean flow, 7-day lowflow, trend in annual mean flow, and trend in 7-day low flow of Elkhorn River at Norfolk, Nebr. 
Table 2-9. Trends in streamflow of North Fork Elkhorn River near Pierce, Nebraska (06799100).

[period analyzed is given as water years; p-value quantifies the probability of the available evidence to conclude for a trend slope different from zero could have arisen when there was actually no temporal trend; $\mathrm{ft}^{3} / \mathrm{s}$, cubic feet per second; <, less than; --, not significant]

\begin{tabular}{|c|c|c|c|c|}
\hline Streamflow statistic & Period analyzed & Kendall's tau & p-value & $\begin{array}{l}\text { Median slope of trend, } \\
\text { in } \mathrm{ft}^{3} / \mathrm{s} \text { per year }(\mathrm{p}<0.05)\end{array}$ \\
\hline \multicolumn{5}{|c|}{ Mean flow } \\
\hline October mean flow & 1961-2004 & -0.025 & 0.808 & -- \\
\hline November mean flow & 1961-2004 & .122 & .245 & -- \\
\hline December mean flow & 1961-2004 & .172 & .099 & -- \\
\hline January mean flow & 1961-2004 & .106 & .311 & -- \\
\hline February mean flow & 1961-2004 & .085 & .418 & -- \\
\hline March mean flow & 1961-2004 & .073 & .485 & -- \\
\hline April mean flow & 1961-2004 & .111 & .288 & -- \\
\hline May mean flow & 1961-2004 & .212 & .042 & 3.6 \\
\hline June mean flow & 1961-2004 & .066 & .531 & -- \\
\hline July mean flow & 1961-2004 & .145 & .166 & -- \\
\hline August mean flow & 1961-2004 & .040 & .700 & -- \\
\hline September mean flow & 1961-2004 & -.023 & .824 & -- \\
\hline Annual mean flow & 1960-2003 & .136 & .202 & -- \\
\hline \multicolumn{5}{|c|}{ Low flow } \\
\hline 1-day low flow & 1961-2004 & .065 & .543 & -- \\
\hline 2-day low flow & 1961-2004 & .063 & .558 & -- \\
\hline 3-day low flow & 1961-2004 & .047 & .668 & -- \\
\hline 7-day low flow & 1961-2004 & .040 & .714 & -- \\
\hline 14-day low flow & 1961-2004 & .040 & .714 & -- \\
\hline 30-day low flow & 1961-2004 & .011 & .925 & -- \\
\hline 60-day low flow & 1961-2004 & .034 & .754 & -- \\
\hline 90-day low flow & 1961-2004 & .045 & .675 & -- \\
\hline 183-day low flow & 1961-2004 & .081 & .451 & -- \\
\hline 1-day low flow (Oct-Nov) & 1959-2003 & .042 & .693 & -- \\
\hline 2-day low flow (Oct-Nov) & 1959-2003 & .037 & .731 & -- \\
\hline 3-day low flow (Oct-Nov) & 1959-2003 & .026 & .808 & -- \\
\hline 7-day low flow (Oct-Nov) & 1959-2003 & -.006 & .960 & -- \\
\hline 14-day low flow (Oct-Nov) & 1959-2003 & -.019 & .863 & -- \\
\hline 30-day low flow (Oct-Nov) & 1959-2003 & -.005 & .968 & -- \\
\hline 60-day low flow (Oct-Nov) & $1959-2003$ & .044 & .678 & -- \\
\hline
\end{tabular}


06799100 North Fork Elkhorn River near Pierce, Nebraska

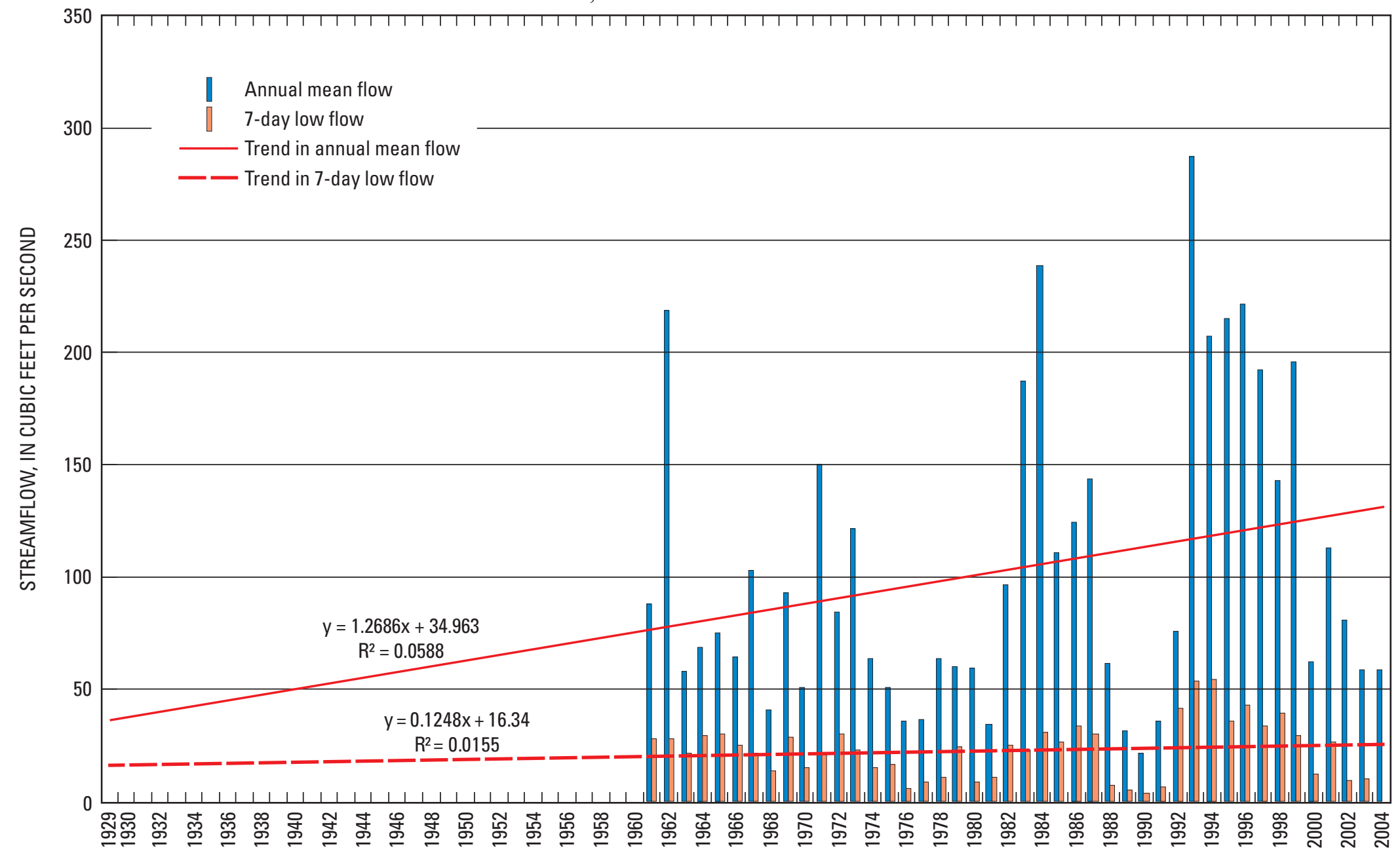

Figure 2-9. Annual mean flow, 7-day lowflow, trend in annual mean flow, and trend in 7-day low flow of North Fork Elkhorn River near Pierce, Nebr. 
Table 2-10. Trends in streamflow of Elkhorn River at West Point, Nebraska (06799350).

[period analyzed is given as water years; $\mathrm{p}$-value quantifies the probability of the available evidence to conclude for a trend slope different from zero could have arisen when there was actually no temporal trend; $\mathrm{ft}^{3} / \mathrm{s}$, cubic feet per second; <, less than; --, not significant]

\begin{tabular}{|c|c|c|c|c|}
\hline Streamflow statistic & Period analyzed & Kendall's tau & p-value & $\begin{array}{l}\text { Median slope of trend, } \\
\text { in } \mathrm{ft}^{3} / \mathrm{s} \text { per year }(\mathrm{p}<0.05)\end{array}$ \\
\hline \multicolumn{5}{|c|}{ Mean flow } \\
\hline October mean flow & 1973-2004 & 0.230 & 0.065 & -- \\
\hline November mean flow & 1973-2004 & .258 & .038 & 13 \\
\hline December mean flow & 1973-2004 & .335 & .007 & 16 \\
\hline January mean flow & 1973-2004 & .250 & .044 & 8.4 \\
\hline February mean flow & 1973-2004 & .133 & .284 & -- \\
\hline March mean flow & 1973-2004 & -.044 & .721 & -- \\
\hline April mean flow & 1973-2004 & .056 & .650 & -- \\
\hline May mean flow & 1973-2004 & .222 & .074 & -- \\
\hline June mean flow & 1973-2004 & .157 & .206 & -- \\
\hline July mean flow & 1973-2004 & .331 & .008 & 35 \\
\hline August mean flow & 1973-2004 & .181 & .144 & -- \\
\hline September mean flow & 1973-2004 & .210 & .092 & -- \\
\hline Annual mean flow & 1973-2003 & .209 & .103 & -- \\
\hline \multicolumn{5}{|c|}{ Low flow } \\
\hline 1-day low flow & 1974-2004 & .155 & .227 & -- \\
\hline 2-day low flow & 1974-2004 & .151 & .241 & -- \\
\hline 3-day low flow & 1974-2004 & .163 & .202 & -- \\
\hline 7-day low flow & 1974-2004 & .204 & .110 & -- \\
\hline 14-day low flow & 1974-2004 & .191 & .135 & -- \\
\hline 30-day low flow & 1974-2004 & .260 & .041 & 7.2 \\
\hline 60-day low flow & 1974-2004 & .320 & .012 & 9.1 \\
\hline 90-day low flow & 1974-2004 & .295 & .021 & 9.8 \\
\hline 183-day low flow & 1974-2004 & .316 & .013 & 9.7 \\
\hline 1-day low flow (Oct-Nov) & $1972-2003$ & .185 & .140 & -- \\
\hline 2-day low flow (Oct-Nov) & $1972-2003$ & .179 & .154 & -- \\
\hline 3-day low flow (Oct-Nov) & $1972-2003$ & .202 & .108 & -- \\
\hline 7-day low flow (Oct-Nov) & $1972-2003$ & .242 & .054 & -- \\
\hline 14-day low flow (Oct-Nov) & $1972-2003$ & .242 & .054 & -- \\
\hline 30-day low flow (Oct-Nov) & $1972-2003$ & .262 & .036 & 8.3 \\
\hline 60-day low flow (Oct-Nov) & $1972-2003$ & .242 & .054 & -- \\
\hline
\end{tabular}




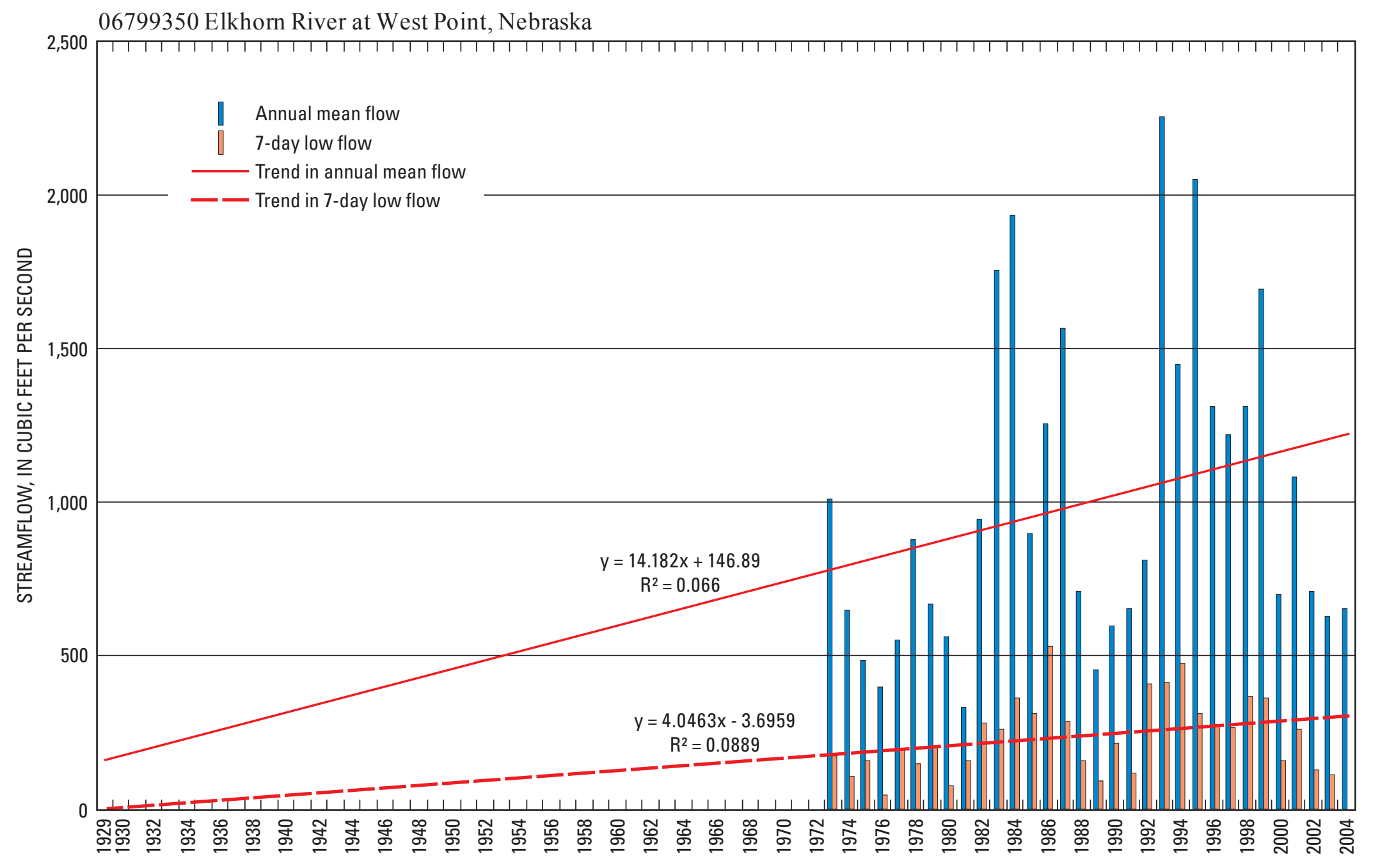

Figure 2-10. Annual mean flow, 7-day lowflow, trend in annual mean flow, and trend in 7-day low flow of Elkhorn River at West Point, Nebr. 
Table 2-11. Trends in streamflow of Pebble Creek at Scribner, Nebraska (06799385).

[period analyzed is given as water years; p-value quantifies the probability of the available evidence to conclude for a trend slope different from zero could have arisen when there was actually no temporal trend; $\mathrm{ft}^{3} / \mathrm{s}$, cubic feet per second; <, less than; --, not significant]

\begin{tabular}{|c|c|c|c|c|}
\hline Streamflow statistic & Period analyzed & Kendall's tau & p-value & $\begin{array}{l}\text { Median slope of trend, } \\
\text { in } \mathrm{ft}^{3} / \mathrm{s} \text { per year }(p<0.05)\end{array}$ \\
\hline \multicolumn{5}{|c|}{ Mean flow } \\
\hline October mean flow & $1980-2004$ & -0.382 & 0.102 & -- \\
\hline November mean flow & 1980-2004 & -.164 & .484 & -- \\
\hline December mean flow & $1980-2004$ & -.491 & .036 & -2.3 \\
\hline January mean flow & 1980-2004 & -.273 & .243 & -- \\
\hline February mean flow & $1980-2004$ & -.491 & .036 & -3.8 \\
\hline March mean flow & 1980-2004 & -.091 & .697 & -- \\
\hline April mean flow & 1980-2004 & .018 & .938 & -- \\
\hline May mean flow & $1980-2004$ & .200 & .392 & -- \\
\hline June mean flow & 1980-2004 & -.273 & .243 & -- \\
\hline July mean flow & 1980-2004 & -.382 & .102 & -- \\
\hline August mean flow & 1980-2004 & -.527 & .024 & -18 \\
\hline September mean flow & 1980-2004 & -.564 & .016 & -5.0 \\
\hline Annual mean flow & 1979-2003 & .053 & .726 & -- \\
\hline \multicolumn{5}{|c|}{ Low flow } \\
\hline 1-day low flow & $1980-2004$ & .287 & .047 & 0.5 \\
\hline 2-day low flow & $1980-2004$ & .293 & .042 & .5 \\
\hline 3-day low flow & $1980-2004$ & .297 & .040 & .5 \\
\hline 7-day low flow & $1980-2004$ & .320 & .027 & .5 \\
\hline 14-day low flow & 1980-2004 & .327 & .023 & .6 \\
\hline 30-day low flow & 1980-2004 & .413 & .004 & .8 \\
\hline 60-day low flow & 1980-2004 & .287 & .047 & .7 \\
\hline 90-day low flow & $1980-2004$ & .240 & .097 & -- \\
\hline 183-day low flow & $1980-2004$ & .127 & .388 & -- \\
\hline 1-day low flow (Oct-Nov) & $1978-2003$ & .323 & .022 & .8 \\
\hline 2-day low flow (Oct-Nov) & $1978-2003$ & .317 & .024 & .8 \\
\hline 3-day low flow (Oct-Nov) & $1978-2003$ & .314 & .026 & .8 \\
\hline 7-day low flow (Oct-Nov) & $1978-2003$ & .262 & .064 & -- \\
\hline 14-day low flow (Oct-Nov) & $1978-2003$ & .243 & .086 & -- \\
\hline 30-day low flow (Oct-Nov) & $1978-2003$ & .212 & .134 & -- \\
\hline 60-day low flow (Oct-Nov) & $1978-2003$ & .138 & .332 & -- \\
\hline
\end{tabular}




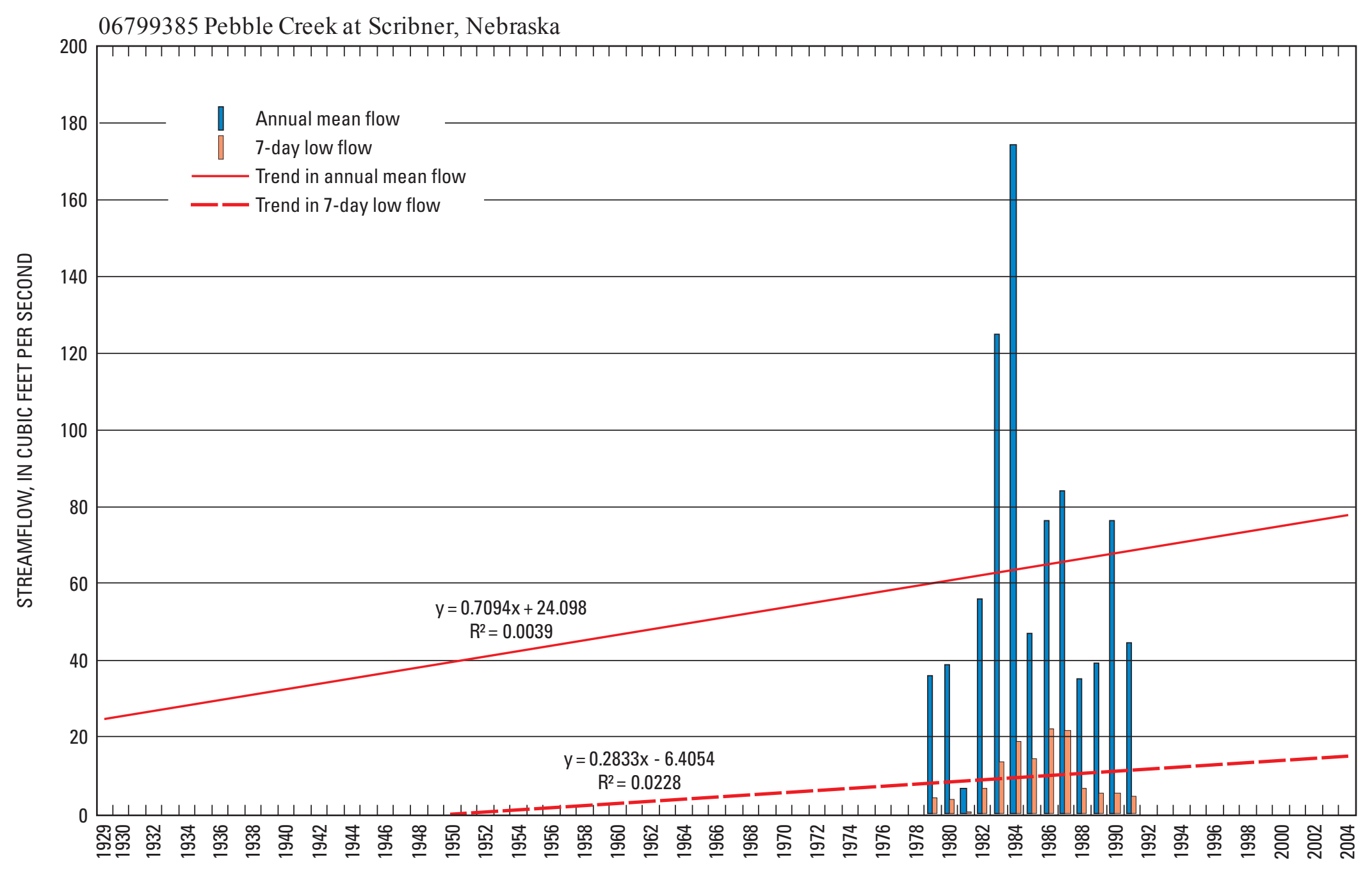

Figure 2-11. Annual mean flow, 7-day lowflow, trend in annual mean flow, and trend in 7-day low flow of Pebble Creek at Scribner, Nebr. 
Table 2-12. Trends in streamflow of Logan Creek near Uehling, Nebraska (06799500).

[period analyzed is given as water years; $p$-value quantifies the probability of the available evidence to conclude for a trend slope different from zero could have arisen when there was actually no temporal trend; $\mathrm{ft}^{3} / \mathrm{s}$, cubic feet per second; <, less than; --, not significant; NC, slope not calculated]

\begin{tabular}{|c|c|c|c|c|}
\hline Streamflow statistic & Period analyzed & Kendall's tau & p-value & $\begin{array}{l}\text { Median slope of trend, } \\
\text { in } \mathrm{ft}^{3} / \mathrm{s} \text { per year }(\mathrm{p}<0.05)\end{array}$ \\
\hline \multicolumn{5}{|c|}{ Mean flow } \\
\hline October mean flow & $1941-2004$ & 0.351 & $<0.000$ & $\mathrm{NC}$ \\
\hline November mean flow & $1941-2004$ & .446 & $<.000$ & $\mathrm{NC}$ \\
\hline December mean flow & $1941-2004$ & .501 & $<.000$ & $\mathrm{NC}$ \\
\hline January mean flow & $1941-2004$ & .474 & $<.000$ & $\mathrm{NC}$ \\
\hline February mean flow & $1941-2004$ & .238 & .005 & $\mathrm{NC}$ \\
\hline March mean flow & $1941-2004$ & .103 & .226 & $\mathrm{NC}$ \\
\hline April mean flow & 1941-2004 & .305 & $<.000$ & $\mathrm{NC}$ \\
\hline May mean flow & 1941-2004 & .317 & $<.000$ & $\mathrm{NC}$ \\
\hline June mean flow & $1941-2004$ & .180 & .034 & $\mathrm{NC}$ \\
\hline July mean flow & $1941-2004$ & .202 & .017 & $\mathrm{NC}$ \\
\hline August mean flow & $1941-2004$ & .181 & .033 & $\mathrm{NC}$ \\
\hline September mean flow & 1941-2004 & .300 & $<.000$ & $\mathrm{NC}$ \\
\hline Annual mean flow & $1941-2003$ & .285 & .001 & $\mathrm{NC}$ \\
\hline \multicolumn{5}{|c|}{ Low flow } \\
\hline 1-day low flow & $1942-2004$ & .475 & $<.001$ & 1.4 \\
\hline 2-day low flow & 1942-2004 & .457 & $<.001$ & 1.3 \\
\hline 3-day low flow & $1942-2004$ & .458 & $<.001$ & 1.3 \\
\hline 7-day low flow & $1942-2004$ & .445 & $<.001$ & 1.4 \\
\hline 14-day low flow & $1942-2004$ & .438 & $<.001$ & 1.4 \\
\hline 30-day low flow & $1942-2004$ & .462 & $<.001$ & 1.5 \\
\hline 60-day low flow & 1942-2004 & .458 & $<.001$ & 1.6 \\
\hline 90-day low flow & $1942-2004$ & .450 & $<.001$ & 1.8 \\
\hline 183-day low flow & $1942-2004$ & .361 & $<.001$ & 1.8 \\
\hline 1-day low flow (Oct-Nov) & $1940-2003$ & .487 & $<.001$ & 1.7 \\
\hline 2-day low flow (Oct-Nov) & $1940-2003$ & .477 & $<.001$ & 1.7 \\
\hline 3-day low flow (Oct-Nov) & $1940-2003$ & .471 & $<.001$ & 1.7 \\
\hline 7-day low flow (Oct-Nov) & $1940-2003$ & .467 & $<.001$ & 1.6 \\
\hline 14-day low flow (Oct-Nov) & $1940-2003$ & .434 & $<.001$ & 1.6 \\
\hline 30-day low flow (Oct-Nov) & $1940-2003$ & .420 & $<.001$ & 1.5 \\
\hline 60-day low flow (Oct-Nov) & $1940-2003$ & .395 & $<.001$ & 1.7 \\
\hline
\end{tabular}




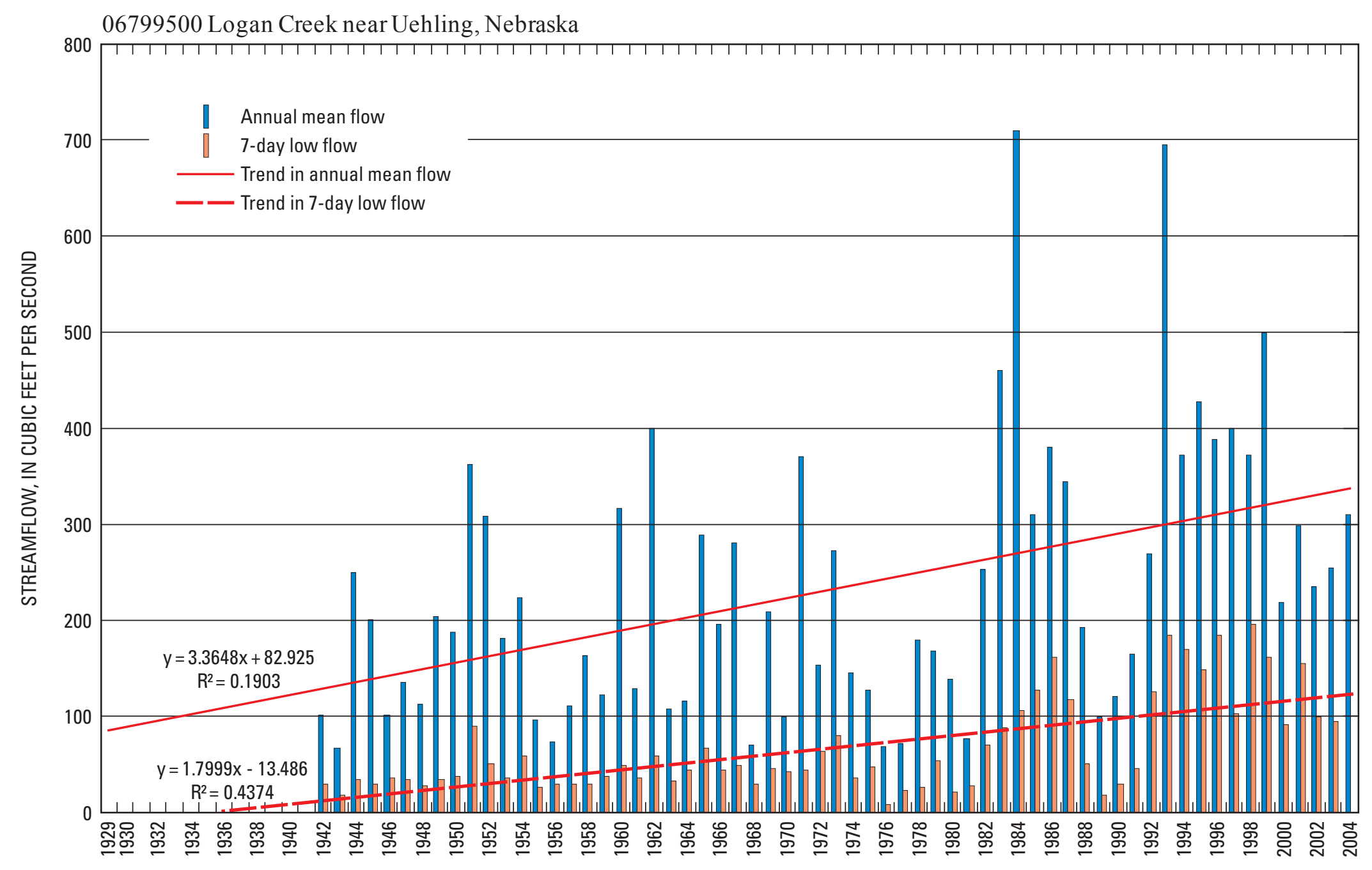

Figure 2-12. Annual mean flow, 7-day lowflow, trend in annual mean flow, and trend in 7-day low flow of Logan Creek near Uehling, Nebr. 
Table 2-13. Trends in streamflow of Maple Creek near Nickerson, Nebraska (06800000).

[period analyzed is given as water years; $p$-value quantifies the probability of the available evidence to conclude for a trend slope different from zero could have arisen when there was actually no temporal trend; $\mathrm{ft}^{3} / \mathrm{s}$, cubic feet per second; $<$, less than; --, not significant]

\begin{tabular}{|c|c|c|c|c|}
\hline Streamflow statistic & Period analyzed & Kendall's tau & p-value & $\begin{array}{l}\text { Median slope of trend, } \\
\text { in } \mathrm{ft}^{3} / \mathrm{s} \text { per year }(\mathrm{p}<0.05)\end{array}$ \\
\hline \multicolumn{5}{|c|}{ Mean flow } \\
\hline October mean flow & $1953-2004$ & 0.229 & 0.016 & 0.9 \\
\hline November mean flow & 1953-2004 & .347 & $<.001$ & 1.2 \\
\hline December mean flow & $1953-2004$ & .342 & $<.001$ & 1.0 \\
\hline January mean flow & 1953-2004 & .306 & .001 & .9 \\
\hline February mean flow & $1953-2004$ & .173 & .068 & -- \\
\hline March mean flow & $1953-2004$ & .165 & .080 & -- \\
\hline April mean flow & 1953-2004 & .241 & .011 & 1.5 \\
\hline May mean flow & 1953-2004 & .261 & .006 & 2.5 \\
\hline June mean flow & 1953-2004 & .118 & .214 & -- \\
\hline July mean flow & 1953-2004 & .231 & .014 & 3.4 \\
\hline August mean flow & $1953-2004$ & .198 & .036 & 1.6 \\
\hline September mean flow & 1953-2004 & .258 & .006 & .9 \\
\hline Annual mean flow & $1952-2003$ & .275 & .004 & 1.2 \\
\hline \multicolumn{5}{|c|}{ Low flow } \\
\hline 1-day low flow & $1953-2004$ & .345 & $<.001$ & .2 \\
\hline 2-day low flow & 1953-2004 & .341 & $<.001$ & .2 \\
\hline 3-day low flow & 1953-2004 & .334 & $<.001$ & .2 \\
\hline 7-day low flow & 1953-2004 & .321 & .001 & .2 \\
\hline 14-day low flow & 1953-2004 & .318 & .001 & .3 \\
\hline 30-day low flow & $1953-2004$ & .345 & $<.001$ & .4 \\
\hline 60-day low flow & $1953-2004$ & .360 & $<.001$ & .6 \\
\hline 90-day low flow & 1953-2004 & .380 & $<.001$ & .6 \\
\hline 183-day low flow & 1953-2004 & .311 & .001 & .7 \\
\hline 1-day low flow (Oct-Nov) & $1951-2003$ & .294 & .002 & .4 \\
\hline 2-day low flow (Oct-Nov) & $1951-2003$ & .292 & .002 & .4 \\
\hline 3-day low flow (Oct-Nov) & $1951-2003$ & .291 & .002 & .4 \\
\hline 7-day low flow (Oct-Nov) & $1951-2003$ & .279 & .003 & .4 \\
\hline 14-day low flow (Oct-Nov) & $1951-2003$ & .271 & .004 & .4 \\
\hline 30-day low flow (Oct-Nov) & $1951-2003$ & .274 & .004 & .5 \\
\hline 60-day low flow (Oct-Nov) & $1951-2003$ & .293 & .002 & 1.7 \\
\hline
\end{tabular}




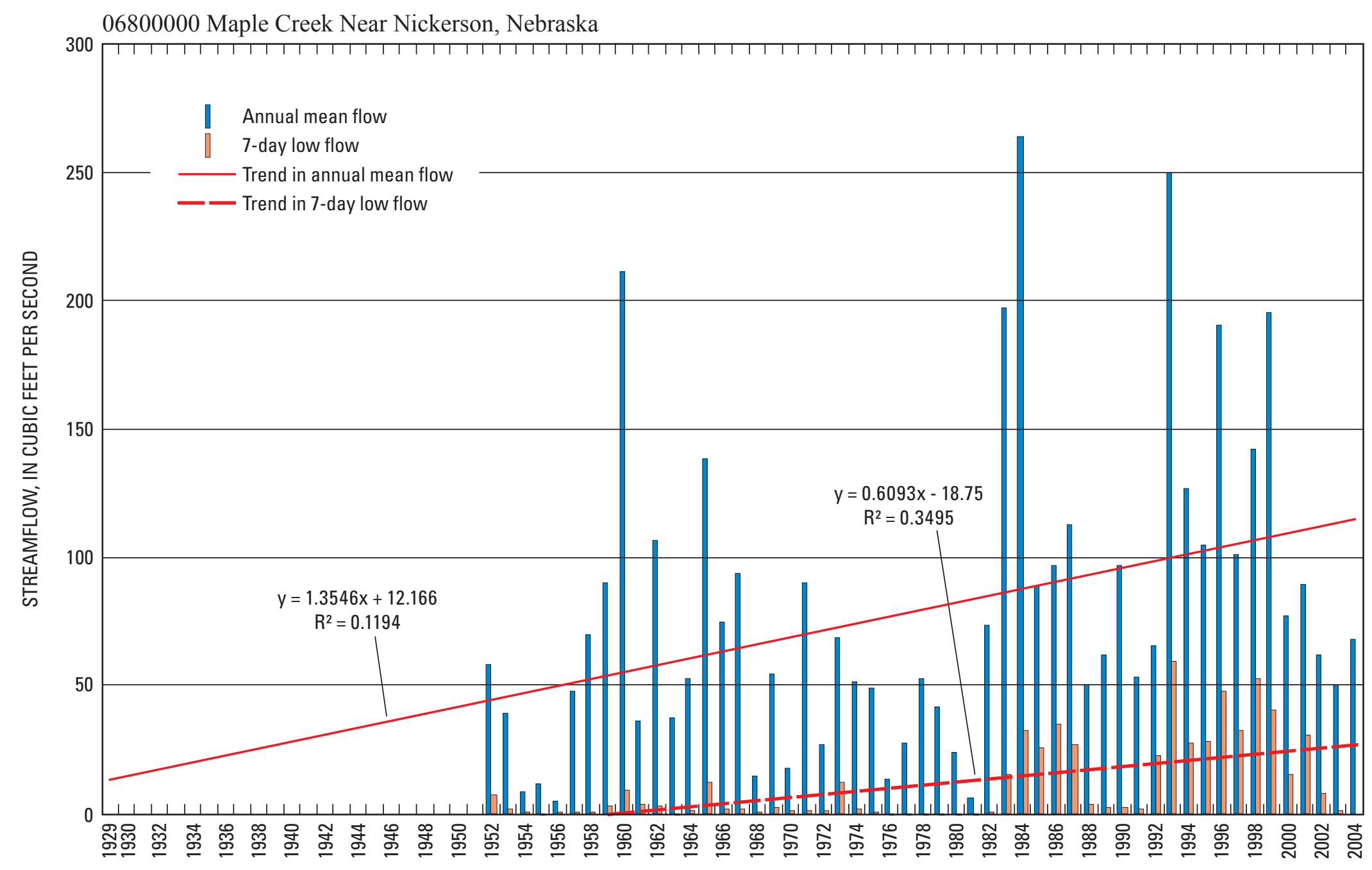

Figure 2-13. Annual mean flow, 7-day lowflow, trend in annual mean flow, and trend in 7-day low flow of Maple Creek Near Nickerson, Nebr. 
Table 2-14. Trends in streamflow of Elkhorn River at Waterloo, Nebraska (06800500).

[period analyzed is given as water years; p-value quantifies the probability of the available evidence to conclude for a trend slope different from zero could have arisen when there was actually no temporal trend; $\mathrm{ft}^{3} / \mathrm{s}$, cubic feet per second; <, less than; --, not significant]

\begin{tabular}{|c|c|c|c|c|}
\hline Streamflow statistic & Period analyzed & Kendall's tau & p-value & $\begin{array}{l}\text { Median slope of trend, } \\
\text { in } \mathrm{ft}^{3} / \mathrm{s} \text { per year }(\mathrm{p}<0.05)\end{array}$ \\
\hline \multicolumn{5}{|c|}{ Mean flow } \\
\hline October mean flow & 1929-2004 & 0.301 & $<0.001$ & 10 \\
\hline November mean flow & 1929-2004 & .392 & $<.001$ & 11 \\
\hline December mean flow & 1929-2004 & .431 & $<.001$ & 11 \\
\hline January mean flow & 1929-2004 & .397 & $<.001$ & 9.3 \\
\hline February mean flow & 1929-2004 & .353 & $<.001$ & 17 \\
\hline March mean flow & 1929-2004 & .204 & .009 & 18 \\
\hline April mean flow & 1929-2004 & .315 & $<.001$ & 29 \\
\hline May mean flow & 1929-2004 & .303 & $<.001$ & 27 \\
\hline June mean flow & 1929-2004 & .196 & .012 & 22 \\
\hline July mean flow & $1928-2004$ & .256 & .001 & 22 \\
\hline August mean flow & 1929-2004 & .180 & .021 & 9.3 \\
\hline September mean flow & 1929-2004 & .201 & .010 & 7.9 \\
\hline Annual mean flow & $1928-2003$ & .341 & $<.001$ & 15 \\
\hline \multicolumn{5}{|c|}{ Low flow } \\
\hline 1-day low flow & 1929-2004 & .383 & $<.001$ & 4.1 \\
\hline 2-day low flow & 1929-2004 & .385 & $<.001$ & 4.3 \\
\hline 3-day low flow & 1929-2004 & .381 & $<.001$ & 4.4 \\
\hline 7-day low flow & 1929-2004 & .355 & $<.001$ & 4.8 \\
\hline 14-day low flow & 1929-2004 & .361 & $<.001$ & 5.4 \\
\hline 30-day low flow & 1929-2004 & .363 & $<.001$ & 6.0 \\
\hline 60-day low flow & 1929-2004 & .380 & $<.001$ & 6.6 \\
\hline 90-day low flow & 1929-2004 & .398 & $<.001$ & 7.1 \\
\hline 183-day low flow & 1929-2004 & .393 & .000 & 7.4 \\
\hline 1-day low flow (Oct-Nov) & $1927-2003$ & .381 & $<.001$ & 5.6 \\
\hline 2-day low flow (Oct-Nov) & $1927-2003$ & .382 & $<.001$ & 5.5 \\
\hline 3-day low flow (Oct-Nov) & $1927-2003$ & .376 & $<.001$ & 5.5 \\
\hline 7-day low flow (Oct-Nov) & $1927-2003$ & .376 & $<.001$ & 6.1 \\
\hline 14-day low flow (Oct-Nov) & $1927-2003$ & .352 & $<.001$ & 6.3 \\
\hline 30-day low flow (Oct-Nov) & $1927-2003$ & .331 & $<.001$ & 6.2 \\
\hline 60-day low flow (Oct-Nov) & $1927-2003$ & .358 & $<.001$ & 7.8 \\
\hline
\end{tabular}




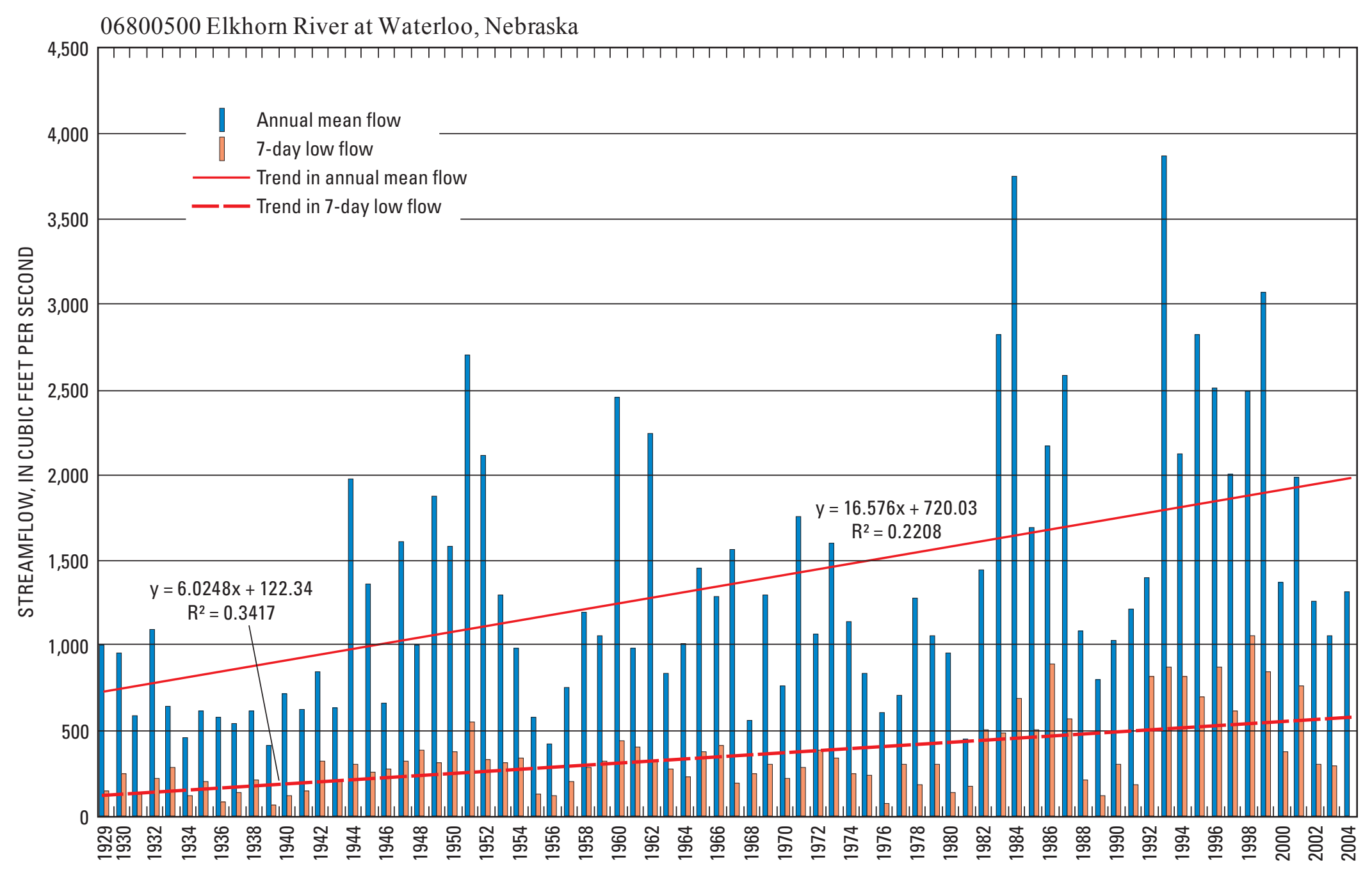

Figure 2-14. Annual mean flow, 7-day lowflow, trend in annual mean flow, and trend in 7-day low flow of Elkhorn River at Waterloo, Nebr. 
Table 2-15. Trends in streamflow of Salt Creek at Greenwood, Nebraska (06803555).

[period analyzed is given as water years; p-value quantifies the probability of the available evidence to conclude for a trend slope different from zero could have arisen when there was actually no temporal trend; $\mathrm{ft}^{3} / \mathrm{s}$, cubic feet per second; <, less than; --, not significant]

\begin{tabular}{|c|c|c|c|c|}
\hline Streamflow statistic & Period analyzed & Kendall's tau & p-value & $\begin{array}{l}\text { Median slope of trend, } \\
\text { in } \mathrm{ft}^{3} / \mathrm{s} \text { per year }(\mathrm{p}<0.05)\end{array}$ \\
\hline \multicolumn{5}{|c|}{ Mean flow } \\
\hline October mean flow & $1953-2004$ & 0.257 & 0.007 & 2.5 \\
\hline November mean flow & 1953-2004 & .472 & $<.001$ & 3.4 \\
\hline December mean flow & $1953-2004$ & .478 & $<.001$ & 2.4 \\
\hline January mean flow & 1953-2004 & .380 & $<.001$ & 1.9 \\
\hline February mean flow & 1953-2004 & .141 & .137 & -- \\
\hline March mean flow & $1953-2004$ & .102 & .283 & -- \\
\hline April mean flow & 1953-2004 & .191 & .044 & 3.0 \\
\hline May mean flow & $1953-2004$ & .277 & .003 & 11 \\
\hline June mean flow & $1953-2004$ & .131 & .165 & -- \\
\hline July mean flow & 1953-2004 & .159 & .093 & -- \\
\hline August mean flow & 1953-2004 & .101 & .286 & -- \\
\hline September mean flow & 1953-2004 & .145 & .125 & -- \\
\hline Annual mean flow & $1952-2003$ & .198 & .039 & 2.9 \\
\hline \multicolumn{5}{|c|}{ Low flow } \\
\hline 1-day low flow & $1953-2004$ & .560 & $<.001$ & 1.7 \\
\hline 2-day low flow & 1953-2004 & .541 & $<.001$ & 1.7 \\
\hline 3-day low flow & $1953-2004$ & .526 & $<.001$ & 1.7 \\
\hline 7-day low flow & $1953-2004$ & .498 & $<.001$ & 1.7 \\
\hline 14-day low flow & $1953-2004$ & .488 & $<.001$ & 1.7 \\
\hline 30-day low flow & $1953-2004$ & .480 & $<.001$ & 2.0 \\
\hline 60-day low flow & $1953-2004$ & .495 & $<.001$ & 2.0 \\
\hline 90-day low flow & $1953-2004$ & .498 & $<.001$ & 2.0 \\
\hline 183-day low flow & 1953-2004 & .419 & .000 & 2.2 \\
\hline 1-day low flow (Oct-Nov) & $1951-2003$ & .449 & $<.001$ & 1.5 \\
\hline 2-day low flow (Oct-Nov) & $1951-2003$ & .446 & $<.001$ & 1.5 \\
\hline 3-day low flow (Oct-Nov) & $1951-2003$ & .435 & $<.001$ & 1.5 \\
\hline 7-day low flow (Oct-Nov) & $1951-2003$ & .429 & $<.001$ & 1.5 \\
\hline 14-day low flow (Oct-Nov) & $1951-2003$ & .428 & $<.001$ & 1.7 \\
\hline 30-day low flow (Oct-Nov) & $1951-2003$ & .463 & $<.001$ & 2.0 \\
\hline 60-day low flow (Oct-Nov) & $1951-2003$ & .391 & $<.001$ & 2.6 \\
\hline
\end{tabular}


06803555 Salt Creek at Greenwood, Nebraska

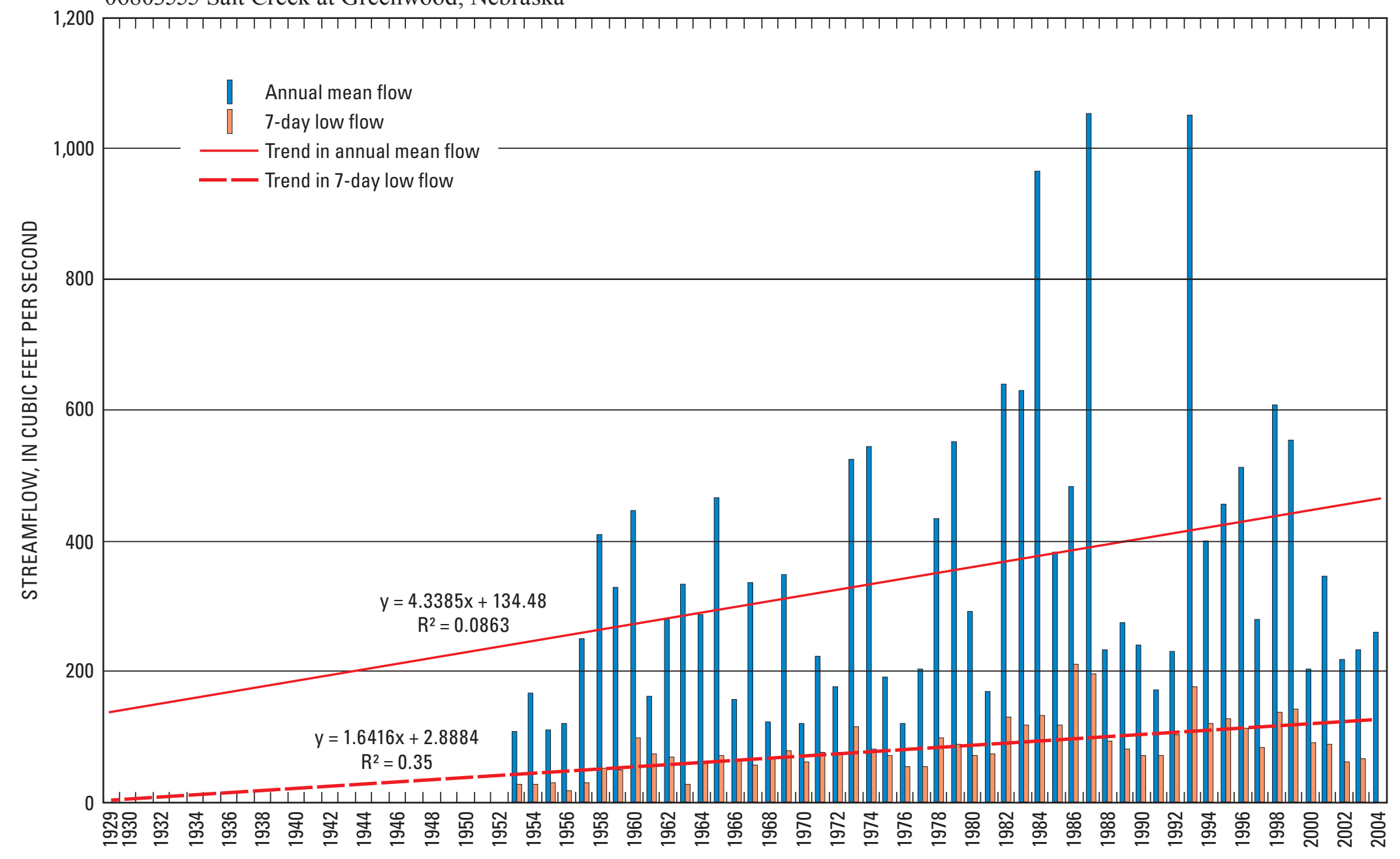

Figure 2-15. Annual mean flow, 7-day lowflow, trend in annual mean flow, and trend in 7-day low flow of Salt Creek at Greenwood, Nebr. 
Table 2-16. Trends in streamflow of Wahoo Creek at Ashland, Nebraska (06804700).

[period analyzed is given as water years; p-value quantifies the probability of the available evidence to conclude for a trend slope different from zero could have arisen when there was actually no temporal trend; $\mathrm{ft}^{3} / \mathrm{s}$, cubic feet per second; <, less than; --, not significant]

\begin{tabular}{|c|c|c|c|c|}
\hline Streamflow statistic & Period analyzed & Kendall's tau & p-value & $\begin{array}{l}\text { Median slope of trend, } \\
\text { in } \mathrm{ft}^{3} / \mathrm{s} \text { per year }(p<0.05)\end{array}$ \\
\hline \multicolumn{5}{|c|}{ Mean flow } \\
\hline October mean flow & $1991-2005$ & -0.181 & 0.347 & -- \\
\hline November mean flow & $1991-2005$ & -.181 & .347 & -- \\
\hline December mean flow & $1991-2005$ & -.210 & .276 & -- \\
\hline January mean flow & $1991-2005$ & -.295 & .125 & -- \\
\hline February mean flow & $1991-2005$ & -.086 & .656 & -- \\
\hline March mean flow & $1991-2005$ & -.276 & .151 & -- \\
\hline April mean flow & $1991-2005$ & -.181 & .347 & -- \\
\hline May mean flow & $1991-2005$ & .067 & .729 & -- \\
\hline June mean flow & $1991-2005$ & -.276 & .151 & -- \\
\hline July mean flow & $1991-2005$ & -.581 & .003 & -28 \\
\hline August mean flow & $1991-2005$ & -.276 & .151 & -- \\
\hline September mean flow & $1991-2005$ & -.371 & .054 & -- \\
\hline Annual mean flow & 1990-2004 & -.333 & .127 & -- \\
\hline \multicolumn{5}{|c|}{ Low flow } \\
\hline 1-day low flow & $1991-2005$ & -.090 & .714 & -- \\
\hline 2-day low flow & $1991-2005$ & -.090 & .714 & -- \\
\hline 3-day low flow & $1991-2005$ & -.090 & .714 & -- \\
\hline 7-day low flow & $1991-2005$ & -.103 & .669 & -- \\
\hline 14-day low flow & $1991-2005$ & -.103 & .669 & -- \\
\hline 30-day low flow & $1991-2005$ & -.154 & .502 & -- \\
\hline 60-day low flow & $1991-2005$ & -.179 & .428 & -- \\
\hline 90-day low flow & $1991-2005$ & -.179 & .428 & -- \\
\hline 183-day low flow & $1991-2005$ & -.282 & .200 & -- \\
\hline 1-day low flow (Oct-Nov) & 1989-2004 & -.229 & .254 & -- \\
\hline 2-day low flow (Oct-Nov) & 1989-2004 & -.219 & .275 & -- \\
\hline 3-day low flow (Oct-Nov) & 1989-2004 & -.210 & .298 & -- \\
\hline 7-day low flow (Oct-Nov) & 1989-2004 & -.200 & .322 & -- \\
\hline 14-day low flow (Oct-Nov) & 1989-2004 & -.200 & .322 & -- \\
\hline 30-day low flow (Oct-Nov) & 1989-2004 & -.257 & .198 & -- \\
\hline 60-day low flow (Oct-Nov) & 1989-2004 & -.219 & .276 & -- \\
\hline
\end{tabular}




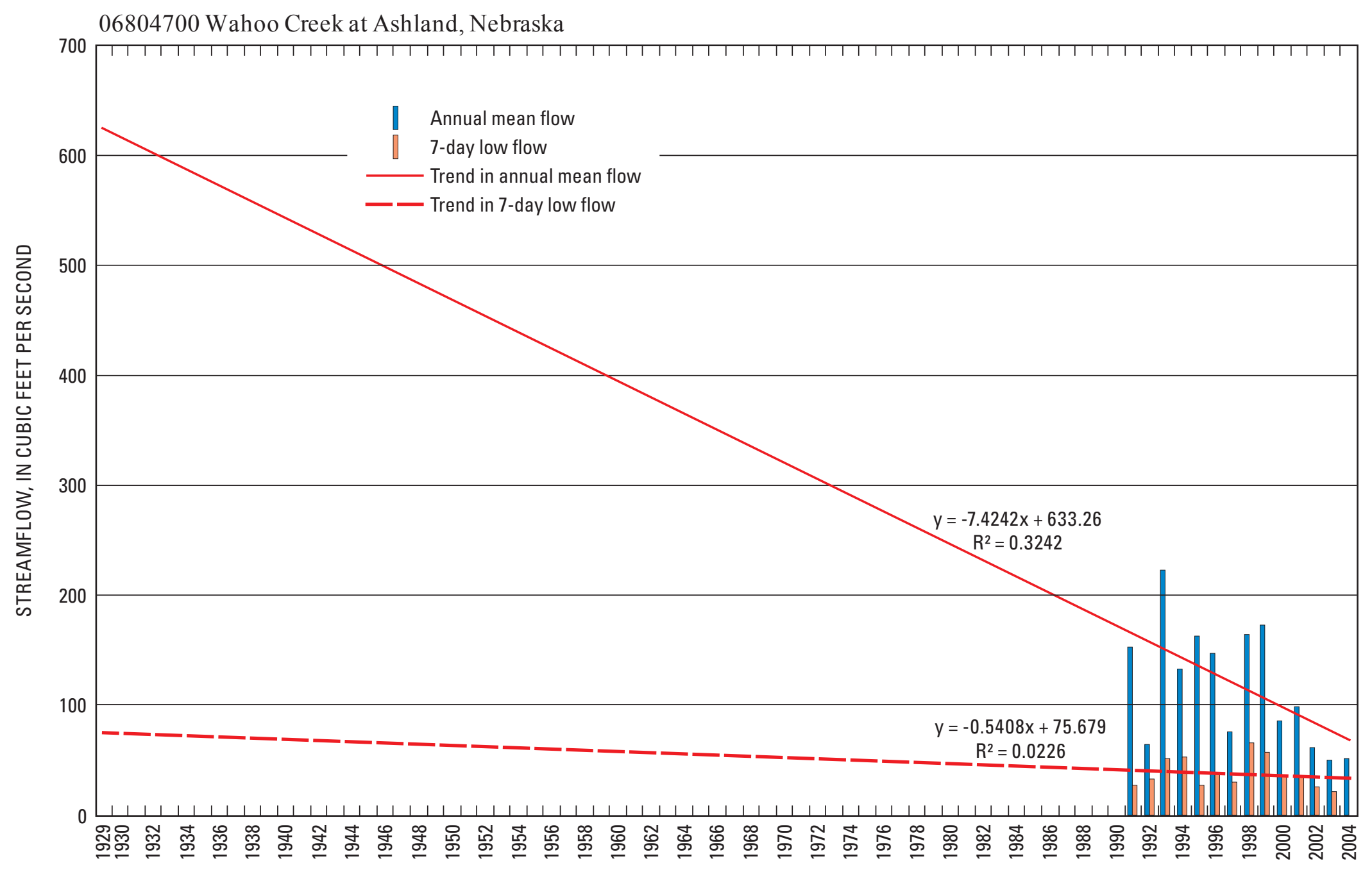

Figure 2-16. Annual mean flow, 7-day lowflow, trend in annual mean flow, and trend in 7-day low flow of Wahoo Creek at Ashland, Nebr. 
Table 2-17. Trends in streamflow of Platte River at Louisville, Nebraska (06805500).

[period analyzed is given as water years; p-value quantifies the probability of the available evidence to conclude for a trend slope different from zero could have arisen when there was actually no temporal trend; $\mathrm{ft}^{3} / \mathrm{s}$, cubic feet per second; <, less than; --, not significant]

\begin{tabular}{|c|c|c|c|c|}
\hline Streamflow statistic & Period analyzed & Kendall's tau & $\mathrm{p}$-value & $\begin{array}{l}\text { Median slope of trend, } \\
\text { in } \mathrm{ft}^{3} / \mathrm{s} \text { per year }(\mathrm{p}<0.05)\end{array}$ \\
\hline \multicolumn{5}{|c|}{ Mean flow } \\
\hline October mean flow & 1954-2004 & 0.229 & 0.016 & 63 \\
\hline November mean flow & $1954-2004$ & .347 & $<.001$ & 63 \\
\hline December mean flow & 1954-2004 & .342 & $<.001$ & 66 \\
\hline January mean flow & 1954-2004 & .306 & .001 & 68 \\
\hline February mean flow & 1954-2004 & .173 & .068 & -- \\
\hline March mean flow & 1954-2004 & .165 & .080 & -- \\
\hline April mean flow & 1954-2004 & .241 & .011 & 72 \\
\hline May mean flow & 1954-2004 & .261 & .006 & 100 \\
\hline June mean flow & 1954-2004 & .118 & .214 & -- \\
\hline July mean flow & 1954-2004 & .256 & .001 & 96 \\
\hline August mean flow & 1954-2004 & .180 & .021 & 57 \\
\hline September mean flow & $1954-2004$ & .201 & .010 & 56 \\
\hline Annual mean flow & 1954-2004 & .282 & .004 & 70 \\
\hline \multicolumn{5}{|c|}{ Low flow } \\
\hline 1-day low flow & 1954-2004 & .344 & $<.001$ & 24 \\
\hline 2-day low flow & 1954-2004 & .354 & $<.001$ & 27 \\
\hline 3-day low flow & 1954-2004 & .334 & .001 & 27 \\
\hline 7-day low flow & 1954-2004 & .291 & .003 & 28 \\
\hline 14-day low flow & 1954-2004 & .252 & .010 & 35 \\
\hline 30-day low flow & 1954-2004 & .260 & .008 & 45 \\
\hline 60-day low flow & 1954-2004 & .267 & .006 & 54 \\
\hline 90-day low flow & 1954-2004 & .276 & .005 & 57 \\
\hline 183-day low flow & 1954-2004 & .314 & .001 & 59 \\
\hline 1-day low flow (Oct-Nov) & $1952-2003$ & .357 & $<.001$ & 53 \\
\hline 2-day low flow (Oct-Nov) & $1952-2003$ & .357 & $<.001$ & 54 \\
\hline 3-day low flow (Oct-Nov) & $1952-2003$ & .351 & $<.001$ & 55 \\
\hline 7-day low flow (Oct-Nov) & $1952-2003$ & .365 & $<.001$ & 58 \\
\hline 14-day low flow (Oct-Nov) & $1952-2003$ & .322 & .001 & 57 \\
\hline 30-day low flow (Oct-Nov) & $1952-2003$ & .311 & .001 & 63 \\
\hline 60-day low flow (Oct-Nov) & $1952-2003$ & .315 & .001 & 69 \\
\hline
\end{tabular}


06805500 Platte River at Louisville, Nebraska

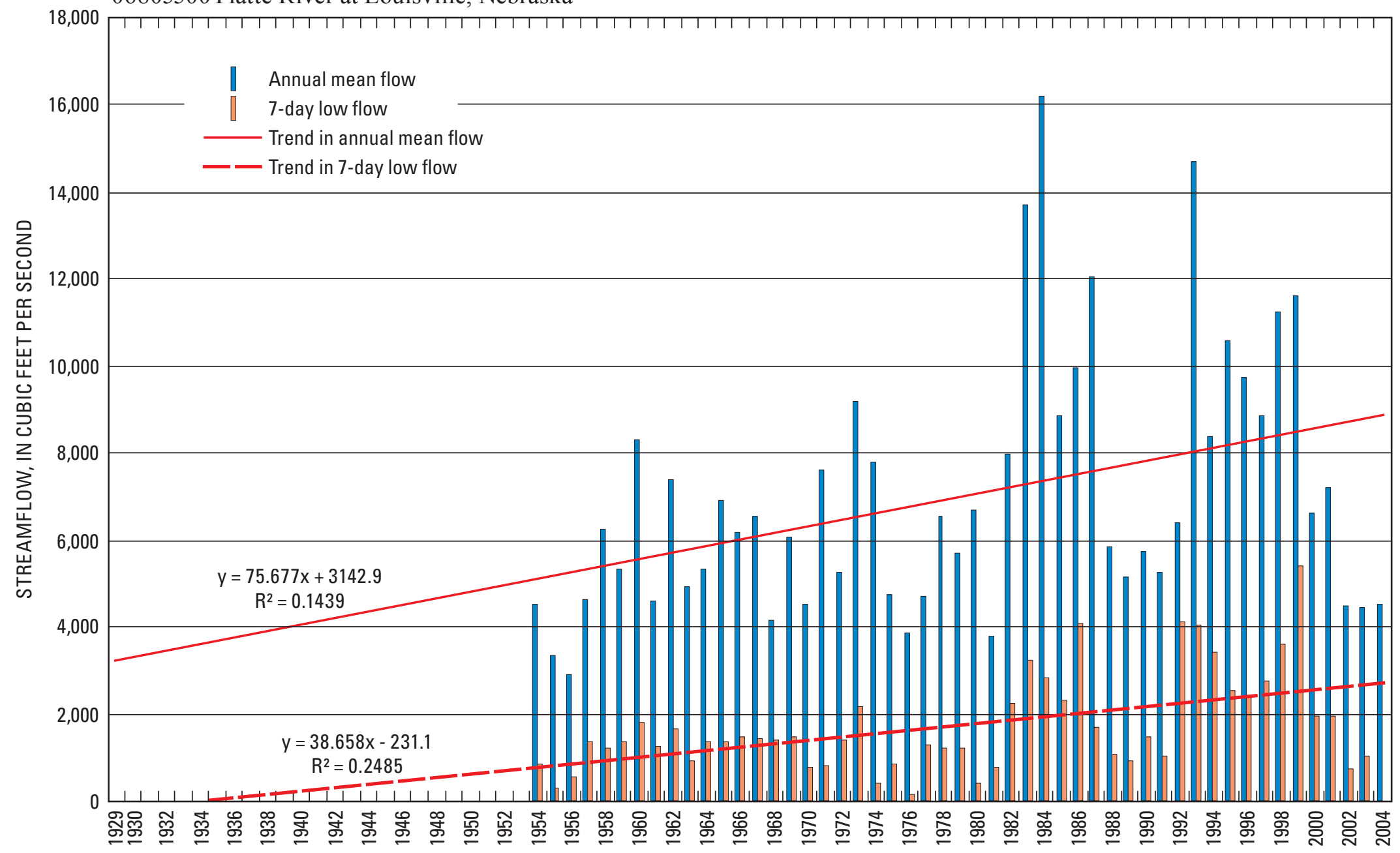

Figure 2-17. Annual mean flow, 7-day lowflow, trend in annual mean flow, and trend in 7-day low flow of Platte River at Louisville, Nebr. 
Publishing support provided by:

Rolla Publishing Service Center

For more information concerning this publication, contact: Director, USGS Nebraska Water Science Center

5231 South 19 Street

Lincoln, NE 68512

(402) 328-4100

Or visit the Nebraska Water Science Center Web site at: http://ne.water.usgs.gov 

